\title{
The Variety of Forced Atmospheric Structure in Response to Tropical SST Anomaly in the Aqua-Planet Experiments
}

\author{
Kensuke NAKAJIMA \\ Faculty of Sciences, Kyushu University, Fukuoka, Japan \\ Yukiko YAMADA \\ Graduate School of Science, Hokkaido University, Sapporo, Japan \\ Yoshiyuki O. TAKAHASHI \\ Center for Planetary Science, Kobe, Japan \\ Faculty of Science, Kobe University, Kobe, Japan \\ Masaki ISHIWATARI \\ Graduate School of Science, Hokkaido University, Sapporo, Japan \\ Wataru OHFUCHI \\ Earth Simulator Center, Japan Agency for Marine-Earth Science and Technology, Yokohama, Japan \\ and \\ Yoshi-Yuki HAYASHI \\ Center for Planetary Science, Kobe, Japan \\ Faculty of Science, Kobe University, Kobe, Japan \\ (Manuscript received 31 October 2011, in final form 22 March 2013)
}

\begin{abstract}
In this paper, we examine the steady state responses of models participating in the Aqua-Planet Experiment Project (APE) to the zonal asymmetry of equatorial sea surface temperature (SST) anomalies (SSTAs). Experiments were performed using three different SSTA distributions, i.e., two localized SSTAs with a common shape but different intensities, and an SSTA that varied with zonal wavenumber one. The structure of the responses obtained differs significantly among the models; however, some common features are also present.

The principal features of the responses to the localized SSTAs are a positive precipitation anomaly over the warm SSTA, a widespread negative precipitation anomaly along the intertropical convergence zone, a pair of Rossby wavetrains along the equatorward flanks of mid-latitude westerly jets originating from a pair of upper tropospheric
\end{abstract}

Corresponding author: Kensuke Nakajima, Faculty of

Sciences, Kyushu University, 6-10-1, Hakozaki, Fukuoka,

Fukuoka 812-8581, Japan

E-mail: kensuke@geo.kyushu-u.ac.jp

(C)2013, Meteorological Society of Japan 
anticyclones that develop to the east of the warm SSTAs, and zonally wavelike precipitation and geopotential anomalies along the baroclinic zones. The structure of the tropical responses differs considerably from the Matsuno-Gill pattern, and the magnitude of the responses is almost proportional to the intensity of the localized SSTA in each of the models.

The responses to the zonal wavenumber one SSTA are dominated by zonal wavenumber one structures. Around the longitudes of the warm (cold) SSTA, tropical precipitation increases (decreases). At longitudes east of the positive precipitation anomaly, the region of nearly zero absolute vorticity near the equator in the upper troposphere expands polewards, and the midlatitude westerly jets become narrower and stronger. To the west of the positive precipitation anomaly, the upper tropospheric region of nearly zero absolute vorticity shrinks, and the mid-latitude jets become weaker but broader, so that the regions of westerly winds extends to the equator, which results in the development of a zonal mean westerly wind anomaly around the equator. The longitudinal shift of the upper tropospheric westerly zonal wind anomaly relative to the precipitation anomaly is in marked contrast to that associated with the Walker circulation and the convection center around the Maritime Continent.

Keywords comparison of atmospheric general circulation models (GCMs); sea surface temperature anomaly (SSTA); equatorial waves; stationary waves; Walker circulation

\section{Introduction}

The general circulation of the atmosphere is driven by the thermal inhomogeneity of the atmosphere itself and that of the ground or ocean surface below. In addition to the planetary-scale meridional thermal contrast caused by the inhomogeneity of solar radiation, there are also zonal contrasts caused by surface inhomogeneities, such as land-sea contrasts and sea surface temperature (SST) variations. Thermal contrasts at the surface drive a variety of zonally inhomogeneous responses in the atmosphere (Webster 1983) such as inhomogeneity of precipitation (Lindzen and Nigam 1987; Neelin and Held 1987), zonally propagating equatorial waves (Matsuno 1966; Gill 1980), and Rossby wavetrains propagating to the extratropics (Bjerkness 1969; Hoskins and Karoly 1981). Inhomogeneity of precipitation is caused, not only directly by the local surface conditions, but also indirectly by remote surface conditions through far-reaching circulation anomalies (Hosaka et al. 1998; Nakajima et al. 2004). These atmospheric responses, in turn, affect the conditions on the ground and sea surface below, all of which develop mutual feedback processes within the land-sea-atmosphere system. An appropriate understanding of such interactions is not only of important theoretical interest, but also indispensable for practical purposes such as weather forecasting and the prediction of future climate change.

With the important roles of the zonal inhomogeneity of surface conditions in mind, the Aqua-Planet Experiment Project (APE) defined three zonally inhomogeneous SST distributions to be specified for the intercomparison of the atmospheric general circulation models (AGCMs). As described by Neale and Hoskins (2000a) and Blackburn and Hoskins (2013) in this special issue, each of these three distributions, $1 \mathrm{KEQ}$, $3 \mathrm{KEQ}$, and $3 \mathrm{KW} 1$, consists of an SST anomaly (SSTA) placed at the equator superimposed on the CONTROL profile, which is one of the zonally homogeneous SST distributions of the APE. In two of the distributions, $1 \mathrm{KEQ}$ and $3 \mathrm{KEQ}$, the SSTAs are localized, whereas in the other, $3 \mathrm{KW} 1$, the SSTA takes the form of the zonal wavenumber one variation. The purpose of these specifications are, as stated by Blackburn and Hoskins (2013): (i) to determine the circulation response to a localized anomaly in tropical SST, the processes that determine the local and global responses, and how these vary between models; and (ii) to determine the circulation response to a planetary-scale anomaly in tropical SST, which involves the generation and propagation of planetary-scale Rossby waves, the longitudinal modulation of extratropical storm tracks, and the impact on meridional transport. All of these issues are among the important interactions that produce the complex behavior of the atmosphere within the climate system (Alexander et al. 2002; Liu and Alexander 2007).

This paper examines the results of AGCM experiments conducted with the zonally varying SSTs in the APE, and aims to identify the similarities and differences in the structure of the atmospheric features that develop as responses to the SSTAs in the 15 participating models. We describe the steady state response of precipitation because it is the primary connection between the tropical SST and the global atmosphere. We also describe and compare the tropical and extratropical dynamical responses, i.e., pressure and wind fields.

We will present rather a large number of figures, 
most of which compare various features of the responses in all of the 15 participating models. By providing these figures, this paper will serve as one of the reference sources for the APE, in particular, for the results of experiments with zonally varying SST. The choice of figures in the present paper is intended to be complementary to the APE-ATLAS (Williamson et al. 2012). The APE-ATLAS contains a large number of figures showing the zonally averaged response to the SST anomalies, the space-time spectra of precipitation at the equator, model mean response structure etc., but the figures showing the responses of individual models are limited.

This paper also intends to complement the APE-ATLAS by providing an explanation of the response structures depicted in the figures. The APE-ATLAS contains a large number of figures, but little description or explanation of those figures. Of course, this is because it aims to be a collection of figures showing the results of the APE project. In this aspect, the present paper can be regarded as an overview of the subset of the APE, which complements the two other overview papers (Blackburn et al. 2013; Williamson et al. 2013) both of which cover the cases with zonally symmetric SSTs.

In due course, a number of unique features and new issues will be presented regarding the description of the APE results derived from a zonally inhomogeneous SST. However, we will not go into these details in the present paper, as here we wish to focus on the complete description of the results of this subset of the APE. Theoretical investigations of those other interesting issues will be left for future studies.

The rest of the paper is organized as follows. Section 2 describes the experimental setup, data, and methods of analysis. In Section 3, the principal features of the atmospheric structures derived from the experiment with zonally homogeneous CONTROL SST profile will be briefly reviewed as this represents the "basic state" of the SSTA experiments. In Section 4, the response to a localized equatorial SST anomaly will be described, mainly from the output of the 3KEQ runs, and in Section 5, the response to zonal wavenumber one variations of SST will be described from the $3 \mathrm{KW} 1$ runs. A summary and concluding remarks will be presented in the last section.

\section{Methods}

\subsection{Specifcation of SST}

The SST in each experiment is prescribed as a function of latitude $(\varphi)$ and longitude $(\lambda)$. In CONTROL experiments, the SST, expressed in degrees Celsius, is zonally uniform and given as:

$$
\begin{aligned}
& T_{\text {Control }}(\lambda, \varphi) \\
& = \begin{cases}27\left[1-\sin ^{2}\left(\frac{90}{60} \varphi\right)\right] & \text { if }|\varphi|<60^{\circ}, \\
0 & \text { if }|\varphi| \geq 60^{\circ} .\end{cases}
\end{aligned}
$$

Neale and Hoskins (2000a) state that the CONTROL SST profile is chosen as the standard "because it leads to a definite, but not unrealistic, single ITCZ regime" in their preliminary experiment. However, as is described in Blackburn et al. (2013), a definite double ITCZ emerges in some of the APE models. The flattening of SST at higher latitudes is introduced to prevent the ocean surface from freezing in the participating models, which are state-of-the-art climate or numerical weather prediction models. In the 1KEQ, 3KEQ, and 3KW1 experiments, SST anomalies are added to the CONTROL SST given above, which are:

$$
\begin{aligned}
& T_{1 \mathrm{KEQ}}(\lambda, \varphi) \\
& = \begin{cases}\cos ^{2}\left(\frac{90}{15} \varphi\right) \cos ^{2}\left(\frac{90}{30} \lambda\right) & \text { if }|\varphi|<15^{\circ} \text { and }|\lambda|<30^{\circ}, \\
0 & \text { otherwise, }\end{cases} \\
& T_{3 \mathrm{KEQ}}(\lambda, \varphi) \\
& = \begin{cases}3 \cos ^{2}\left(\frac{90}{15} \varphi\right) \cos ^{2}\left(\frac{90}{30} \lambda\right) & \text { if }|\varphi|<15^{\circ} \text { and }|\lambda|<30^{\circ}, \\
0 & \text { otherwise, }\end{cases}
\end{aligned}
$$

and

$$
\begin{aligned}
& T_{3 \mathrm{KW} 1}(\lambda, \varphi) \\
& = \begin{cases}3 \cos ^{2}\left(\frac{90}{30} \varphi\right) \cos (\lambda) & \text { if }|\varphi|<30^{\circ}, \\
0 & \text { otherwise, }\end{cases}
\end{aligned}
$$

respectively. These SST distributions are plotted in Fig. 1.

By comparing 1KEQ or 3KEQ with the CONTROL run, we can examine the response of the global atmosphere to a localized equatorial SSTA, including anomalous precipitation, and equatorial and extratropical wave activities that develop mainly in response to the latent heating in the precipitation anomaly. By comparison between 1KEQ and 3KEQ, we can obtain an indication of how linearly the atmosphere responds to an imposed SSTA. The comparison between $3 \mathrm{KW} 1$ and CONTROL should provide information on atmospheric responses to planetary-scale zonal variations of tropical SST.

Also plotted in Fig. 1 is another zonally uniform SST distribution from the APE setup, QOBS, whose latitudinal profile is broader than that of CONTROL. In comparison to QOBS, which is chosen to be "a simple 
(a)

SST: CONTROL and QOBS

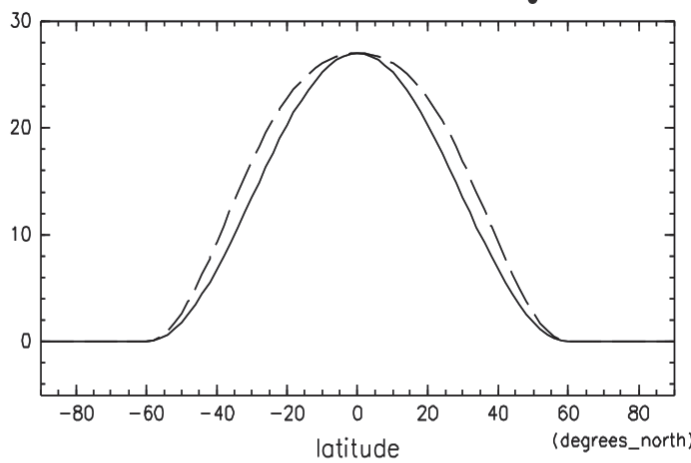

(c)

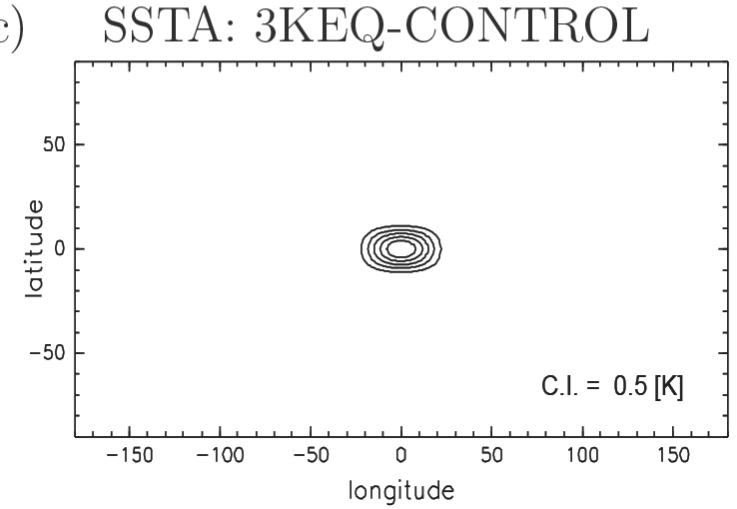

(b)

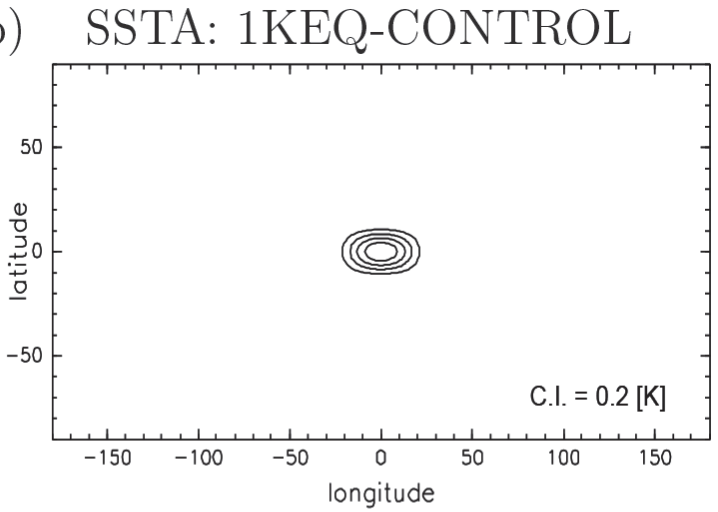

(d)

SSTA: 3KW1-CONTROL

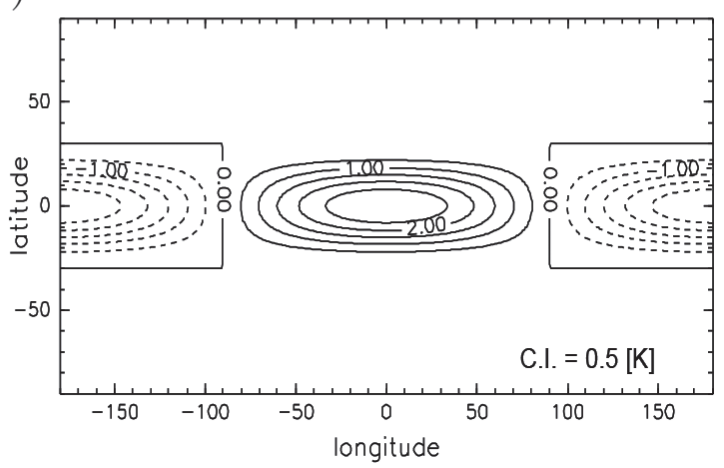

Fig. 1. (a) Specification of SST for the CONTROL (solid) and QOBS (dashed) runs, and SST anomalies from CONTROL for (b) 1KEQ, (c) 3KEQ, and (d) 3KW1. (Units: degrees Celsius).

geometric function closest to the observed zonal mean SST distributions" (Neale and Hoskins 2000a), the characteristics of the CONTROL profile are that the region of high SST is confined around the equator, and the region of large latitudinal gradient of SST are located at lower latitudes. As will be described later, because of these characteristics of the CONTROL profile, the climatological states obtained from the APE runs of the CONTROL experiment are somewhat unusual in all of the participating models compared with those observed in the real atmosphere, and the responses to the SSTAs are greatly influenced by these climatological states.

In some models, there are several differences from the standard specification. First, in the 1KEQ and 3KEQ setups of ECMWF05, the SSTA lacks its western half unintentionally. Second, in the $3 \mathrm{KW} 1$ setup of ECMWF05 and ECMWF07, the meridional scale of the SSTA was unintentionally halved. We chose to include the results of these experiments in this paper, although such deviations for the standard specifications will almost certainly have affected the nature of the response to the SSTA. As will be shown later, these cases display unique responses, and so enrich the variety of the models to be compared. Thirdly, in GFDL, the mean surface pressure is $1000 \mathrm{hPa}$ instead of the standard value of $1013.25 \mathrm{hPa}$. As a result, the GFDL model may exhibit slightly stronger responses to the SSTA than other models would, because a deficit of $1.35 \%$ in the air pressure results in the same level of increase in the water vapor mixing ratio. However, we expect that this small change would not affect the overall features of the responses of the GFDL runs, and so does not affect the intercomparison to be presented here.

\subsection{Data and analysis}

The data analyzed in this paper are the results from the AGCM runs with SST distributions CONTROL, $1 \mathrm{KEQ}, 3 \mathrm{KEQ}$, and $3 \mathrm{KW} 1$ from the APE conducted by 15 participating groups, whose specifications are briefly summarized in Table 1. Unfortunately, the parameterized forcing (PF) data, which was "optional" in the data specification of the APE project, is archived 
Table 1. Participating models

\begin{tabular}{|c|c|c|c|c|c|c|c|}
\hline $\begin{array}{l}\text { model } \\
\text { symbol }\end{array}$ & model & $\begin{array}{l}\text { horizontal } \\
\text { resolution }\end{array}$ & $\begin{array}{l}\text { no. of } \\
\text { levels }\end{array}$ & $\begin{array}{c}\text { deep } \\
\text { convection }\end{array}$ & $\begin{array}{c}\text { short } \\
\text { symbol }\end{array}$ & $\begin{array}{l}\text { PF } \\
\text { data }\end{array}$ & note \\
\hline AGU & AFES & T39 & 48 & Emanuel & $\mathrm{AG}$ & $\bigcirc$ & \\
\hline CGAM & HadAM3 & $3.75^{\circ} \times 2.5^{\circ}$ & 30 & Gregory-Rawntree & CG & & \\
\hline CSIROstd & CCAM-05e & $\sim 210 \mathrm{~km}$ & 18 & McGregor & $\mathrm{CS}$ & & \\
\hline CSIROold & CCAM-05a & $\sim 210 \mathrm{~km}$ & 18 & McGregor & $\mathrm{CO}$ & & \\
\hline DWD & GME & $\sim 1^{\circ}$ & 31 & Tiedtke & DW & 0 & \\
\hline ECMWF05 & IFS cy29r2 & $\mathrm{T} 159$ & 60 & Bechtold et al. 2004 & E5 & $\bigcirc$ & $a, b$ \\
\hline ECMWF07 & IFS cy $32 \mathrm{r} 3$ & $\mathrm{~T} 159$ & 60 & Bechtold et al. 2008 & E7 & $\bigcirc$ & $\mathrm{b}$ \\
\hline GFDL & AM2.1 & $2.5^{\circ} \times 2^{\circ}$ & 24 & RAS & GF & & $\mathrm{c}$ \\
\hline GSFC & NSIPP-1 & $3.75^{\circ} \times 3^{\circ}$ & 34 & RAS & GS & 0 & \\
\hline K1JAPAN & CCSR/NIES 5.7 & T42 & 20 & Pan-Randall & K1 & 0 & \\
\hline LASG & SAMIL & R42 & 9 & Manabe & LA & 0 & \\
\hline MIT & MIT-GCM & $\sim 280 \mathrm{~km}$ & 40 & RAS & MI & & \\
\hline MRI & MRI/JMA98 & T42 & 30 & Randall-Pan & MR & & \\
\hline NCAR & CCSM-CAM3 & $\mathrm{T} 42$ & 26 & Zhang-McFarlane & $\mathrm{NC}$ & 0 & \\
\hline UKMOn96 & pre-HadGAM1 & $1.875^{\circ} \times 1.25^{\circ}$ & 38 & Gregory 1999 & UK & 0 & \\
\hline
\end{tabular}

a. Western half of the 3 KEQ SSTA is lacking.

b. Meridional scale of the $3 \mathrm{KW} 1 \mathrm{SSTA}$ is halved.

c. Mean sea level pressure is $1000 \mathrm{hPa}$.

for a subset of the participating models. Consequently, we were only able to analyze the responses of latent heating due to convective and resolved cloud processes for 9 out of the 15 models. For further details, readers are referred to the APE-ATLAS (Williamson et al. 2012).

All of the materials presented in this paper concern the steady response of variables in the AGCMs to the anomalies of SST defined above. We leave examination of time-dependent responses for future research, including the responses of convectively coupled equatorial waves, the change in the transport properties of mid-latitude baroclinic waves, and the development processes of stationary waves. The anomaly, as the steady response of a particular variable, was calculated for each model as the difference between the temporal mean value of the variable obtained in the particular run of the $1 \mathrm{KEQ}, 3 \mathrm{KEQ}$, or $3 \mathrm{KW} 1 \mathrm{SST}$ profiles, and the temporal and zonal mean value of the variable in the CONTROL experiment of the model. The integration period of each APE run was 3.5 years. Steady state data were obtained by taking the temporal average over the last three years of the time series of each run.

\section{Atmospheric structure in the CONTROL experiment}

To set a suitable context within which the results of the SSTA experiments presented later can be compared, the temporal and zonal mean meridional structures of the CONTROL experiment from the NCAR model are briefly described here. We concentrate on a single model, because the ensemble average of the results from all models would blur dynamically important features. The results of CONTROL experiments obtained from the APE models, and the differences among them, are summarized by Blackburn et al. (2013), and are presented thoroughly in the APE-ATLAS (Williamson et al. 2012). Although the climatological states of the APE models show a significant amount of diversity, even for the temporal and zonal mean structures as described in those references, the following features are typical of the CONTROL runs of the APE models, unless specifically mentioned otherwise.

Figure 2 shows the temporal and zonal mean structure of the atmosphere obtained from the NCAR CONTROL run. The latitudinal profile of precipitation (Fig. 2d) shows a double ITCZ structure at the equator, but the equatorial minimum is not very strong. It should be noted that the overall characteristics of the CONTROL runs are that precipitation tends to be sharply confined around the equator, although the structure of the ITCZ, i.e., single peaked or double peaked, varies considerably among the APE models.

At mid-latitudes, the baroclinic zone shifts equatorward, as does the mid-latitude jet (Figs. 2a, b), compared with their locations in the real atmosphere 
CONTROL Zonal Mean Fields (NCAR)
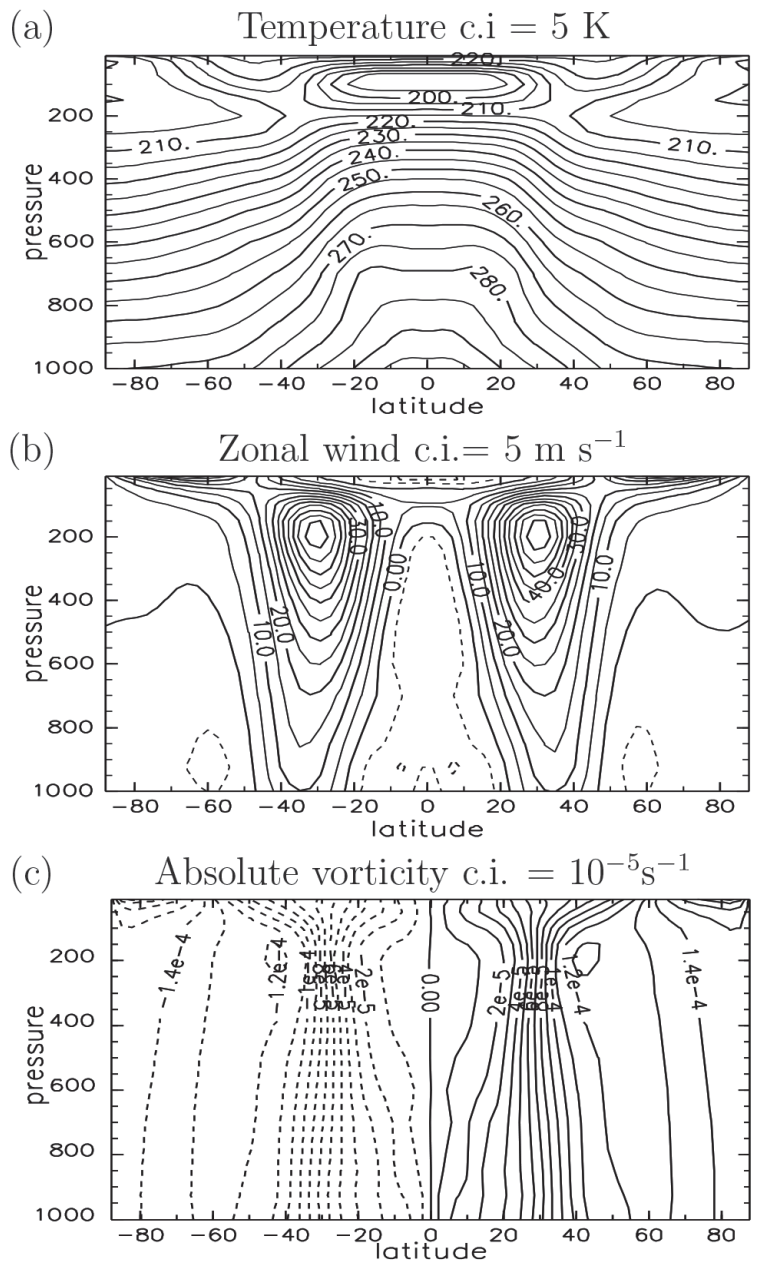

(d)

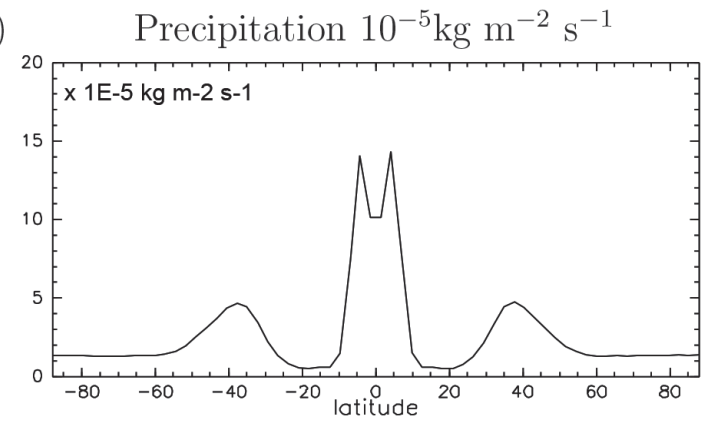

Fig. 2. Time and zonal mean fields obtained from the CONTROL run of NCAR: (a) temperature, (b) zonal wind, (c) absolute vorticity, and (d) precipitation. Units and/or contour interval are indicated at the top of each panel.
(Blackburn et al. 2013; Williamson et al. 2012). Near the surface, the maximum westerly is located a few degrees poleward of the upper jet core latitude (Fig. $2 b)$. Correspondingly, the precipitation maximum associated with the baroclinic waves resides further poleward, at around $40^{\circ}$ latitude (Fig. 2d). We will refer to this single, and intense, tropospheric westerly jet as the mid-latitude jet.

The tropical upper troposphere is strongly influenced by this unusual mid-latitude jet profile. At the $200 \mathrm{hPa}$ level, the mean zonal wind is westerly at $15^{\circ}$ latitude with an intensity of $15 \mathrm{~m} \mathrm{~s}^{-1}$, and exceeds $30 \mathrm{~m} \mathrm{~s}^{-1}$ at $20^{\circ}$ latitude. This deep invasion of the westerly into the tropics is one of the features shared by all of the CONTROL runs of the APE. Note that the term invasion above is used only in relation to the morphology of the westerly jets. Dynamically, the strong westerly in the upper tropical troposphere results from the poleward transport of angular momentum from the equator by the upper branch of the Hadley circulation, as suggested by the nearly homogeneous distribution of absolute vorticity in the upper troposphere equatorward of latitudes around $\pm 15^{\circ}$ (Fig. 2c). This peculiar feature of the zonal wind in the tropics strongly affects many aspects of the general circulation. As a result, some characteristics of the atmosphere, including the response to SSTAs described later, are distinct from those often observed in the real atmosphere.

\section{Response to localized SST anomalies: 1KEQ and 3KEQ}

\subsection{Characteristic features of the responses}

In this section, we compare the results from 15 AGCM runs, mainly for $3 \mathrm{KEQ}$. As the structures of the responses are complex and vary considerably among the models, we will first identify the principal features of the response to the 3KEQ SSTA by focusing on one of the models, GFDL, before considering the wider comparison in an attempt to help readers grasp the nature of the similarities and differences among the responses. In later subsections, we will describe the variety of the responses by pointing out the difference in intensity, location, shape, etc., of the features to be identified here. Subsequently, we will present multimodel statistics for the response of geopotential.

The horizontal distributions of the responses of several atmospheric variables to the 3KEQ SST anomalies from the GFDL model are shown in Figs. 3a to $3 \mathrm{~g}$. Absolute vorticity at $250 \mathrm{hPa}$ is also shown in Fig. $3 \mathrm{~h}$ but will be discussed later. The structures are mostly symmetric about the equator except near the poles. The predominance of a north-south symmetry in 
the response is a common characteristic of the $3 \mathrm{KEQ}$ and 3KW1 runs among all of the 15 APE models. Symmetry degrades in the 1KEQ runs, but it persists at lower latitudes (not shown).

Figure $3 \mathrm{a}$ shows the response of precipitation. There are three latitudinal bands where the response is notable: one is the ITCZ latitudes, while the other two regions are the equatorward flanks of the mid-latitude baroclinic zones at around $30^{\circ}$. In the response at the ITCZ, there are two notable characteristics. First, a strong positive anomaly develops over the prescribed warm SSTA as a direct response to the SSTA, and a negative anomaly prevails at other longitudes. Second, the reduction in precipitation is not zonally uniform; the negative anomaly is weak at some longitudes, $(\lambda \sim$ $-180^{\circ},-140^{\circ},-70^{\circ}, 85^{\circ}$, and $140^{\circ}$ ), and even positive anomalies appear. Similar wave-like zonal variation can also be found in the mixing ratio anomaly at 700 $\mathrm{hPa}$ (Fig. 3b), although the signal is significant, not in the ITCZ, but at about $10^{\circ}$ off the equator. In the responses near the baroclinic zones, the precipitation anomalies also exhibit a wave-like structure; the most notable feature is the appearance of east-west dipoles consisting of positive anomalies centered at $(\lambda, \varphi) \sim$ $\left(60^{\circ}, \pm 32^{\circ}\right)$ the negative anomalies centered at $(\lambda, \varphi) \sim$ $\left(10^{\circ}, \pm 32^{\circ}\right)$. The positive anomaly in the mid-latitudes may be regarded as a generic structure of the increase in rainfall observed in the western United States during the warm events of El Niño (Hoerling and Kumar 2002). At other longitudes, the signature of the precipitation anomaly is generally negative, but some degree of wave-like variation can be found. The anomaly of vertical velocity at $500 \mathrm{hPa}$ (Fig. 3c) exhibits a structure consistent with that of precipitation, i.e., upward (downward) motion in the areas of positive (negative) precipitation anomaly, except that the magnitude of the mid-latitude signal of vertical velocity is more conspicuous than for precipitation.

Figures $3 \mathrm{~d}$ to $3 \mathrm{f}$ show the dynamical response in the middle, lower, and upper troposphere, respectively. One of the most puzzling features is that the equatorial Kelvin wave response expected to the east of the SSTA as a Matsuno-Gill pattern (Matsuno 1966; Gill 1980) seems to be absent or obscured. In the lower troposphere (Fig. 3e), there is no easterly anomaly immediately to the east of the SSTA along the equator; an easterly wind anomaly can be found at longitudes around $60^{\circ} \sim 100^{\circ}$, but it is disconnected from the area of the SSTA. In the upper troposphere (Fig. 3f), there is no westerly anomaly immediately to the east of the SSTA; the upper-level wind anomaly is easterly to the east along the equator, which is contrary to that in the
Kelvin response of the upper level in a Matsuno-Gill pattern. It is also notable that a pair of anticyclones expected to the west in the standard Matsuno-Gill pattern is not significant. At $850 \mathrm{hPa}$, a pair of velocity anomalies in the cyclonic curvature around $(\lambda, \varphi)=$ $\left(-40^{\circ} \sim 0^{\circ}, \pm 10^{\circ}\right)$ may be a trace of the equatorial Rossby wave response to the SSTA. At $250 \mathrm{hPa}$, the pair of anticyclones expected to the west of the SSTA is not present; instead, a pair of anticyclonic wind anomalies develops to the east of the SSTA around $(\lambda, \varphi)=$ $\left(0^{\circ} \sim 20^{\circ}, \pm 20^{\circ}\right)$, which seems to spread widely in the latitudinal direction and to be smoothly connected to the anomalies at higher latitudes. Due to the combination of the eastward shifted Rossby response and apparent absence of the Kelvin response, the upperlevel divergence associated with the enhanced precipitation at the SSTA consists of meridional divergence and zonal convergence, which is contrary to that expected from a Matsuno-Gill pattern in an atmosphere without background wind. In short, the tropical response structure to $3 \mathrm{KEQ}$ is significantly different to the structure expected in the classical linear theory of thermal response to a localized equatorial heat source without background wind. In the upper troposphere, non-linearity becomes important, and the reality of the simple frictional law becomes uncertain. Even in such cases, the longitudinal location of the Rossby response is at the longitude of the SSTA or westwards; it does not develop to the east of the SSTA (Sardeshmukh and Hoskins 1988). The eastward development of the Rossby response in 3KEQ contradicts both the linear and non-linear theories of the thermal response of the equatorial atmosphere. As shown in Appendix A, this apparently strange response can be explained as a result of the unique structure of the zonal mean zonal wind in the CONTROL (and 3KEQ) runs of the APE.

Differences between the response structure in $3 \mathrm{KEQ}$ and that expected from the standard Matsuno-Gill pattern without background wind is also evident in the vertical section at the equator (Fig. 3g). The upward motion and positive temperature anomaly in the area of the positive precipitation anomaly over the SSTA match what is usually expected, although the vertical structure is somewhat complex. However, the response of the zonal wind is quite strange; at low levels, especially to the east of the SSTA, the signal is very weak, and in the middle and upper troposphere, the anomaly is zonally converging. There are two additional unusual features in the temperature response. First, there is a negative temperature anomaly to the west of the SSTA. This may be explained to some extent as a response to the negative precipitation anomaly to the west of 


\section{KEQ Response (GFDL)}

(a) RAIN c.i. $=5 \mathrm{E}-5 \mathrm{~kg} \mathrm{~m}^{-2} \mathrm{~s}^{-1}$

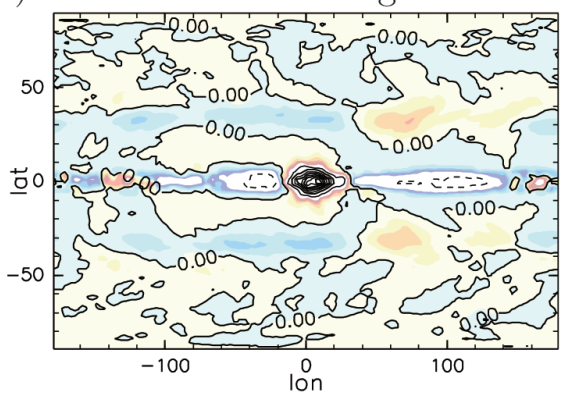

(c)

$\omega 500$ c.i. $=0.01 \mathrm{~Pa} \mathrm{~s}^{-1}$

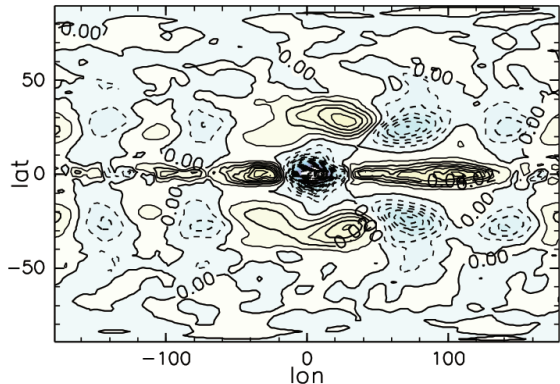

(e) ZUV850 c.i. $=10 \mathrm{~m}$

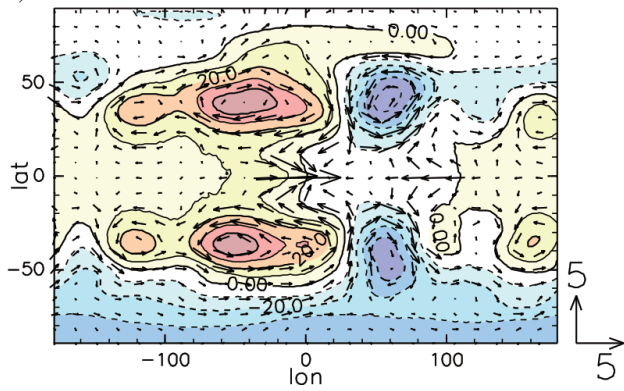

(g) T,U,w at Eq. c.i. $=0.25 \mathrm{~K}$

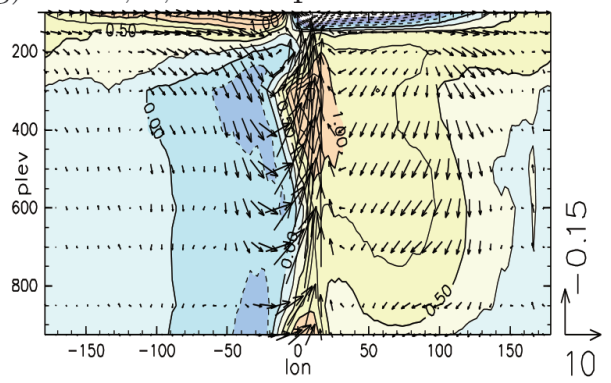

(b)

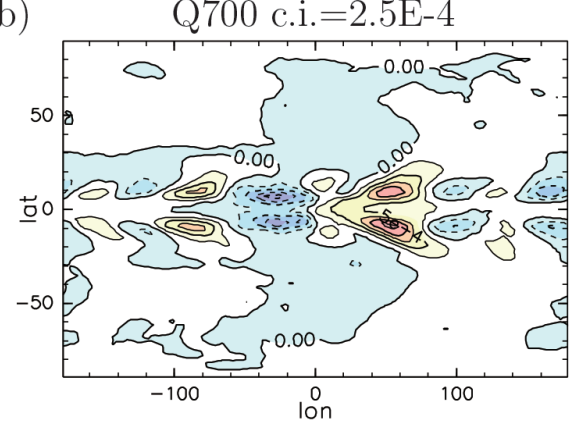

(d)

TUV500 c.i. $=0.4 \mathrm{~K}$

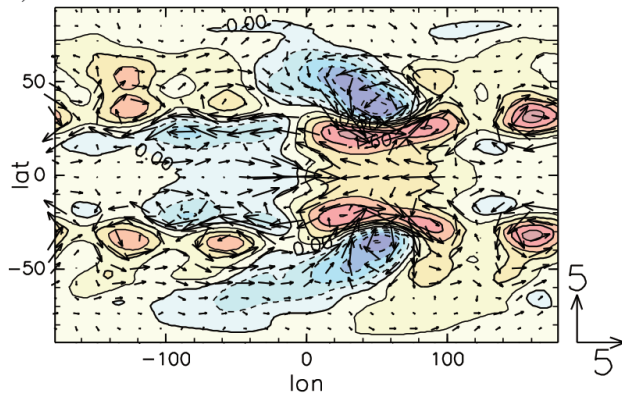

(f)

ZUV250 c.i. $=20 \mathrm{~m}$

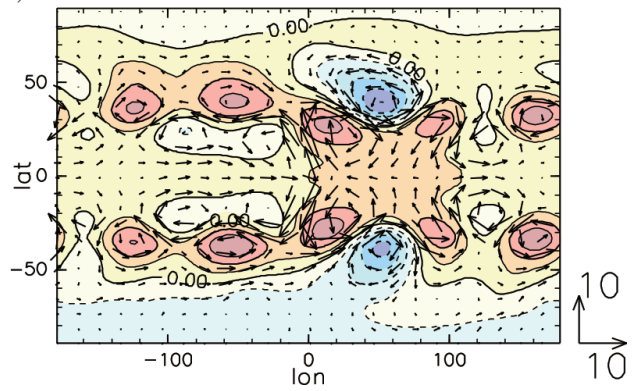

(h) abs.vort.250 c.i. $=1 \mathrm{E}-5$ s-1

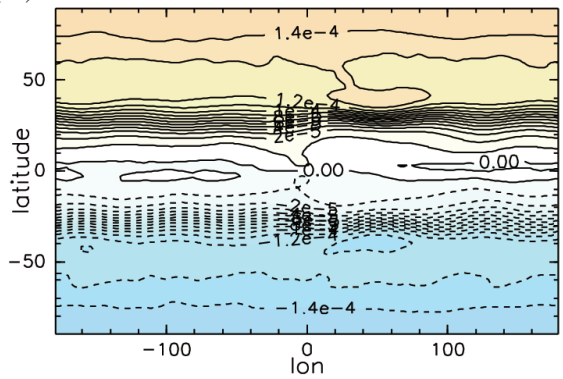

Fig. 3. Time mean response of the 3KEQ run from GFDL. (a) Horizontal distribution of precipitation anomaly. (b) Water vapor mixing ratio anomaly at $700 \mathrm{hPa}$. (c) Pressure velocity anomaly at $500 \mathrm{hPa}$. (d) Temperature and horizontal wind anomalies at $500 \mathrm{hPa}$. (e) Geopotential height and horizontal wind anomalies at $850 \mathrm{hPa}$. (f) Geopotential height and horizontal wind anomalies at $250 \mathrm{hPa}$. (g) Temperature, zonal wind, and vertical $p$-velocity anomalies at the equator. (h) Absolute vorticity at $250 \mathrm{hPa}$. Units and contour intervals are indicated at the top of each panel. Magnitudes of the vector components are indicated to the right of each panel. Units are $\mathrm{m} \mathrm{s}^{-1}$ for horizontal components, and $\mathrm{Pa} \mathrm{s}^{-1}$ for $p$-velocity. 
the SSTA (e.g., Hosaka et al. 1998). Second, there is an area of positive temperature anomaly to the east centered around $(\mathrm{p}, \lambda) \sim\left(600 \mathrm{hPa}, 70^{\circ}\right)$. It is partially detached from the warm anomaly over the SSTA, and, moreover, the vertical structure differs from that of the warm anomaly over the SSTA; the temperature anomaly is most intense in the middle troposphere, where that over the SSTA is at a minimum. This warm anomaly seems to be induced by the deep downward motion at longitudes around $100^{\circ}$ (Figs. 3c, g), which is supported by the meridional convergence in the upper troposphere (Fig. 3f) to the east of the pair of anticyclones.

In the extratropics, a pair of barotropic Rossby wavetrains develops that are notable in the geopotential anomaly of the upper troposphere (Fig. 3f). They emerge as a pair of anticyclones centered at $(\lambda, \varphi)=$ $\left(10^{\circ}, \pm 30^{\circ}\right)$ poleward of the anticyclonic circulations to the east of the SSTA mentioned above, propagate to higher latitudes to appear as a pair of cyclones at $(\lambda, \varphi)=\left(50^{\circ}, \pm 40^{\circ}\right)$, then turn back equatorward to appear as a pair of anticyclones at $(\lambda, \varphi)=\left(90^{\circ}\right.$, $\left.\pm 30^{\circ}\right)$, and then appear as a pair of cyclones at $(\lambda, \varphi)=$ $\left(130^{\circ}, \pm 20^{\circ}\right)$. The Rossby wavetrains seem to continue further eastward to encircle the mid-latitudes meandering in the waveguides at the equatorward flanks of the westerly jets. As is demonstrated in Appendix A, the Rossby wavetrains are excited mainly by the meridional advection of absolute vorticity by the wind anomaly diverging from the site of the positive precipitation anomaly above the SSTA. The vertical structure of the Rossby wavetrains is equivalent barotropic; by comparing Fig. 3e and Fig. 3f, we see that the locations of the cyclonic and anticyclonic geopotential centers coincide throughout the troposphere. It is worth noting that the temperature anomaly is positive (negative) in the high (low) pressure anomalies (Fig. 3d), and hence the height anomaly is more intense in the upper troposphere (Fig. 3e, f). In the lower atmosphere at $850 \mathrm{hPa}$ (Fig. 3e), geopotential anomalies at higher latitudes are more prominent; they are anticyclones at $(\lambda, \varphi)=$ $\left(-60^{\circ}, \pm 40^{\circ}\right)$ and cyclones at $(\lambda, \varphi)=\left(50^{\circ}, \pm 40^{\circ}\right)$.

The zonal wavenumber of the Rossby wavetrains is about 5, which is the same as that of the quasi-stationary features found in the CONTROL experiments (Blackburn et al. 2013; Williamson et al. 2012). Comparing Figs. 3a and 3f, the Rossby wavetrains seem to affect the equatorial anomalies of precipitation; precipitation in the ITCZ seems to be enhanced (suppressed) around the longitudes of mid-latitude anticyclonic (cyclonic) perturbations in the upper troposphere. As is discussed in Section 9 of Blackburn et al. (2013), similar quasi-stationary wave-like variations of precipitation in the ITCZs are identified in most of the CONTROL runs of the APE models. This may imply that the wave-like variation in precipitation found in the 3KEQ runs may not be a response to the SSTA, but is a kind of intrinsic variation that also exists in the CONTROL setup. However, we do not exclude the possibility that this feature is a significant signal caused by the introduction of the SSTA based on two pieces of supporting evidence, i.e., the amplitude of the meridional wind anomalies is larger in the 3KEQ run of GFDL than in the CONTROL run by a factor of about two, and the north-south symmetry is much more distinct than that in the CONTROL run (see Fig. 4.99 of the APE-ATLAS (Williamson et al. 2012)).

\subsection{Variety in the responses of the 15 APE models a. Precipitation}

In the following three subsections, we will compare the responses to the SSTA from the 3KEQ experiment in the 15 APE models. Figure 4 shows the time mean precipitation anomaly. As the response is mostly symmetric about the equator in all of the APE models, the Southern Hemisphere below a latitude of $-15^{\circ}$ is omitted. Latitudes higher than $60^{\circ}$ are also omitted because the precipitation, and its anomaly, is weak.

As for the overall characteristics of the precipitation responses, we can identify all of the corresponding features of the precipitation anomalies mentioned for the GFDL run in the previous subsection, i.e., the intense positive anomaly over the SSTA, the mostly negative anomaly along the ITCZ outside the SSTA, and the east-west dipoles on the equatorward flanks of the baroclinic zones. The wave-like modulation in the tropics and mid-latitudes, presumably related to wavenumber 5 stationary disturbances, can also be seen. However, the detailed structures of the precipitation anomalies are model dependent.

Focusing on the responses in the ITCZ, the positive anomalies over the SSTA for CGAM, CSIROstd, K1JAPAN, and NCAR have two maxima straddling the equator, whereas those for the remaining models have single maxima at the equator. This variation of the meridional profiles seems to reflect those of the ITCZ in the CONTROL runs of the corresponding models, which are presented in Blackburn et al. (2013). The responses of ITCZ precipitation outside the SSTA also exhibit different meridional structures. In CSIROold, DWD, ECMWF05, GFDL, and LASG, single zones of negative precipitation anomalies develop along the equator, while in the other models, negative anomalies are dominant along the latitudinal lines of $\varphi \sim \pm 5^{\circ}$. 
It should be noted that the meridional "double trough" structure of the negative anomaly is not a simple reflection of the structure of the ITCZ in the CONTROL experiment. In CGAM, K1JAPAN, and NCAR, the meridionally double-peaked structures of the precipitation anomalies outside the SSTA in 3KEQ are very distinct, although the double ITCZ structures observed in the CONTROL experiment have rather modest equatorial minima of precipitation (see Fig. 4 in Blackburn et al, 2013). In CGAM, K1JAPAN, and NCAR, zonally averaged anomalies outside the SSTA along the equator are positive. The double-peak structure of the precipitation anomaly also appears in MIT, where the ITCZ in CONTROL is broad, but has a single peak. In short, precipitation becomes focused at the equator to a larger degree in the models where the ITCZs in CONTROL are broad, whether they are single or double. This behavior of precipitation reminds us of the equatorial precipitation enhancement found in the response to a localized equatorial SSTA in Hosaka et al. (1998), where an equatorial Kelvin wave plays an important role in the meridional focusing of precipitation. However, the dynamics of the precipitation response observed in 3KEQ are left for a future study.

Figure 5 and Table 2 show the intensity of the precipitation anomalies in the ITCZs and mid-latitude baroclinic zones from the 15 APE models more quantitatively. In the left column of Fig. 5, the longitudinal distributions of precipitation anomalies along the equator are listed. The peak value over the SSTA varies by a factor of five, from the weakest response in K1JAPAN to the strongest response in ECMWF05. As the longitudinal extent of the SSTA in ECMWF05 is half of the APE specification, as was noted in Section 2, it is expected that the response in ECMWF05 would be stronger still if the SSTA of the specified size were placed. If we compare the anomalies of precipitation by a relative measure, by dividing the precipitation anomaly in 3KEQ by the zonal mean precipitation at the corresponding latitudes in CONTROL for each run, scattering among the models is reduced considerably (the leftmost two columns in Table 2); the maximum values range from $156 \%$ to $333 \%$, and the minimum values are about $70 \%$ of the CONTROL on the equator. As for those models with a double-peak structure, the range of the scaled responses at the off-equatorial peak latitudes are shown in the next two columns of Table 2. Some of those models show a rainfall reduction even larger than half of the CONTROL. Such reductions in precipitation at the off-equatorial peak latitudes typically occur just to the west of the SSTA, as suggested by the distribution of the unscaled precipitation anomaly (Fig. 4)

In the central column of Fig. 5, the longitudinal distributions of the precipitation anomalies, meridionally averaged between $\pm 15^{\circ}$, are plotted from the 15 models. As variability in the meridional structure is mostly eliminated by the meridional averaging, the longitudinal distributions become similar to each other, although a few outliers remain. The scaled responses in the same latitudinal band listed in the 6th and 7th columns of Table 2 also confirm the reduction in scattering among the models; in most of the models, the maximum located on the SSTA is around $250 \%$, and the minimum located to the west of the SSTA is around $70 \%$, of the precipitation in the CONTROL experiment. A similarly significant reduction in precipitation to the west of the SSTA was also found in previous studies (Hosaka et al. 1998; Neale and Hoskins 2000b), and has been interpreted as the result of a Rossby wave response.

In the right column of Fig. 5, the longitudinal distributions of the precipitation anomalies on the southern flank of the mid-latitude baroclinic zone averaged between latitudes of $+20^{\circ}$ and $+40^{\circ}$ are plotted. The dipole shape anomaly, consisting of a reduction at longitudes around $0^{\circ}$ and enhancement at longitudes around $60^{\circ}$, is commonly noted in all of the models, but with varying intensity. The variation associated with wavenumber 5 , noted earlier, can also be identified, and its amplitude varies among the models. In the scaled responses listed in the last two columns of Table 2 , the amplitudes of the mid-latitude average precipitation anomalies are about $20 \%$ of the CONTROL in most of the models.

\section{b. Horizontal structure of dynamic fields}

The horizontal structures of the responses, namely anomalies in the horizontal wind and geopotential height fields at the $250 \mathrm{hPa}$ and $850 \mathrm{hPa}$ levels for all of the 15 models are shown in Figs. 6 to 9. As are for the precipitation anomalies, the responses are mostly symmetric about the equator, so the Southern Hemisphere is omitted.

In general, the features previously identified in GFDL are also evident here: (1) the tropical response that is dissimilar to the Matsuno-Gill pattern; (2) the extratropical equivalent barotropic Rossby wavetrains and wavenumber 5 feature along the baroclinic zone excited by the meridional divergent wind from the positive precipitation anomaly over the SSTA; and (3) the prominent appearance of zonally dipole geopotential anomalies in the higher latitudes at the $850 \mathrm{hPa}$ level. However, the intensities, the horizontal scales, 
3KEQ Precipitation Anomalies

(a) AGU

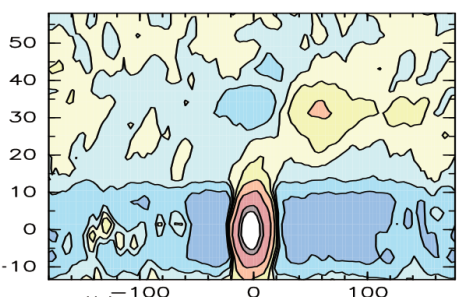

(d) CSIROold

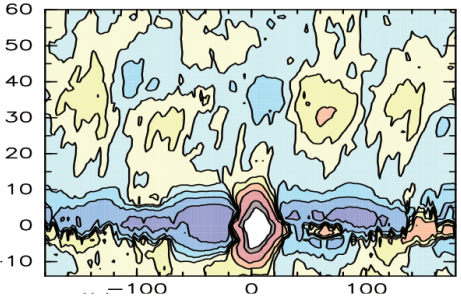

(g) ECMWF07

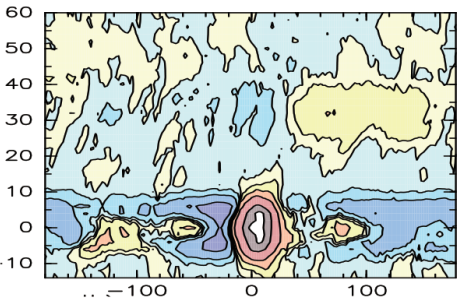

(j) K1JAPAN

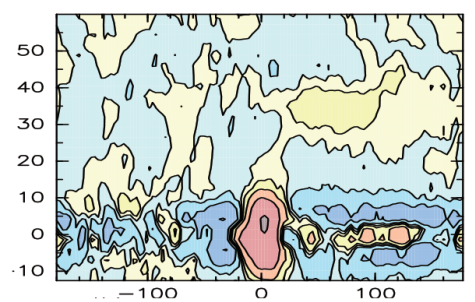

(m) MRI

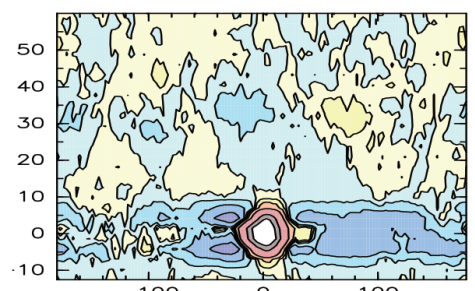

(b) CGAM

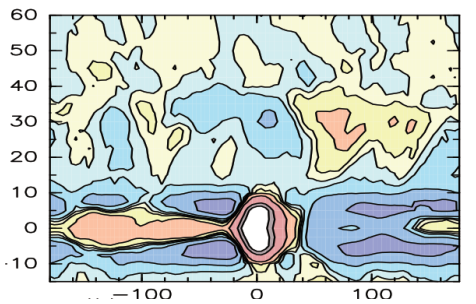

(e) DWD

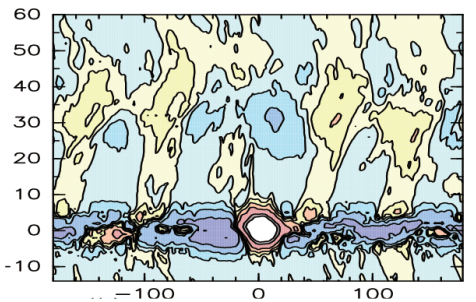

(h) GFDL

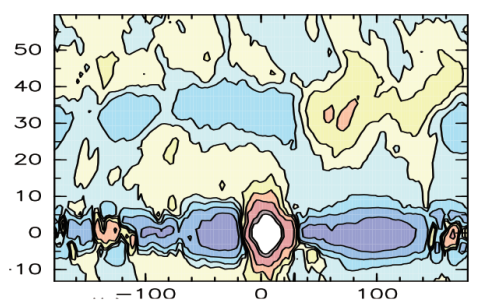

(k) LASG

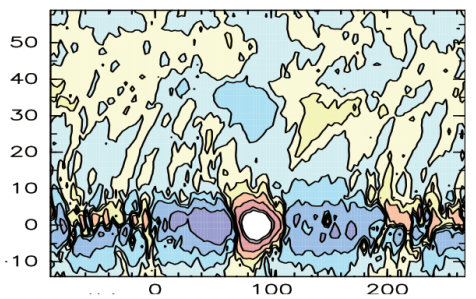

(n) NCAR

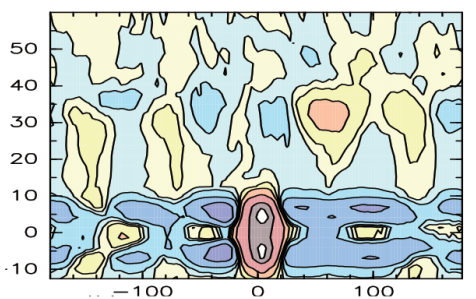

(c) CSIROstd

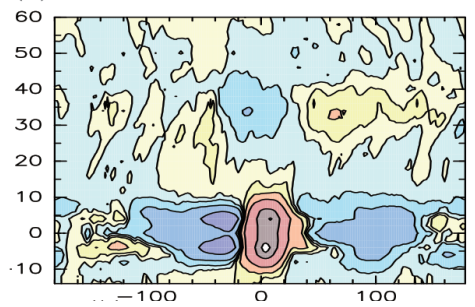

(f) ECMWF05

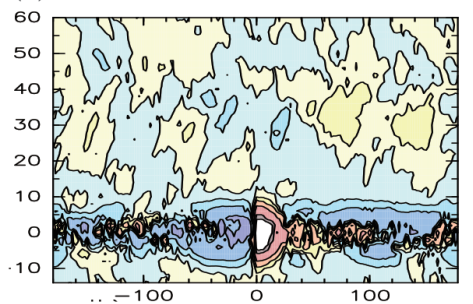

(i) GSFC

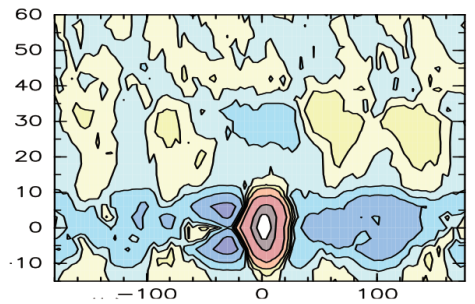

(1) MIT

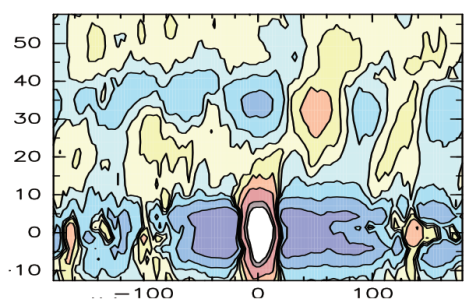

(o) UKMOn96

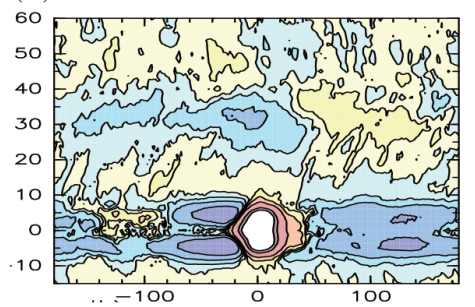

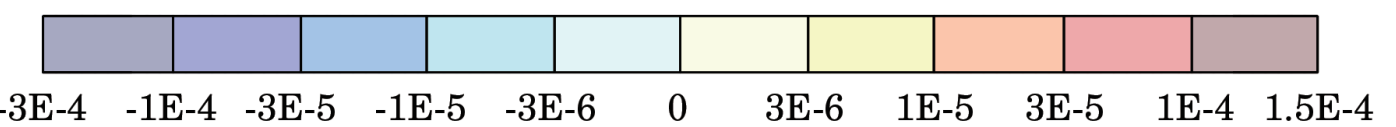

Fig. 4. Time mean precipitation anomalies in the 3 KEQ runs from the 15 APE models. Units: $\mathrm{kg} \mathrm{m}^{-2} \mathrm{~s}^{-1}$. Note that the polar region and most of the Southern Hemisphere are omitted, and also that the region of precipitation greater than $1.5 \times 10^{-4} \mathrm{~kg} \mathrm{~m}^{-2} \mathrm{~s}^{-1}$ is not shaded to highlight the precipitation maximum around the SSTA. See Table 1 for the label legends. 


\section{KEQ Responses of Precipitation in Selected Latitude Bands}
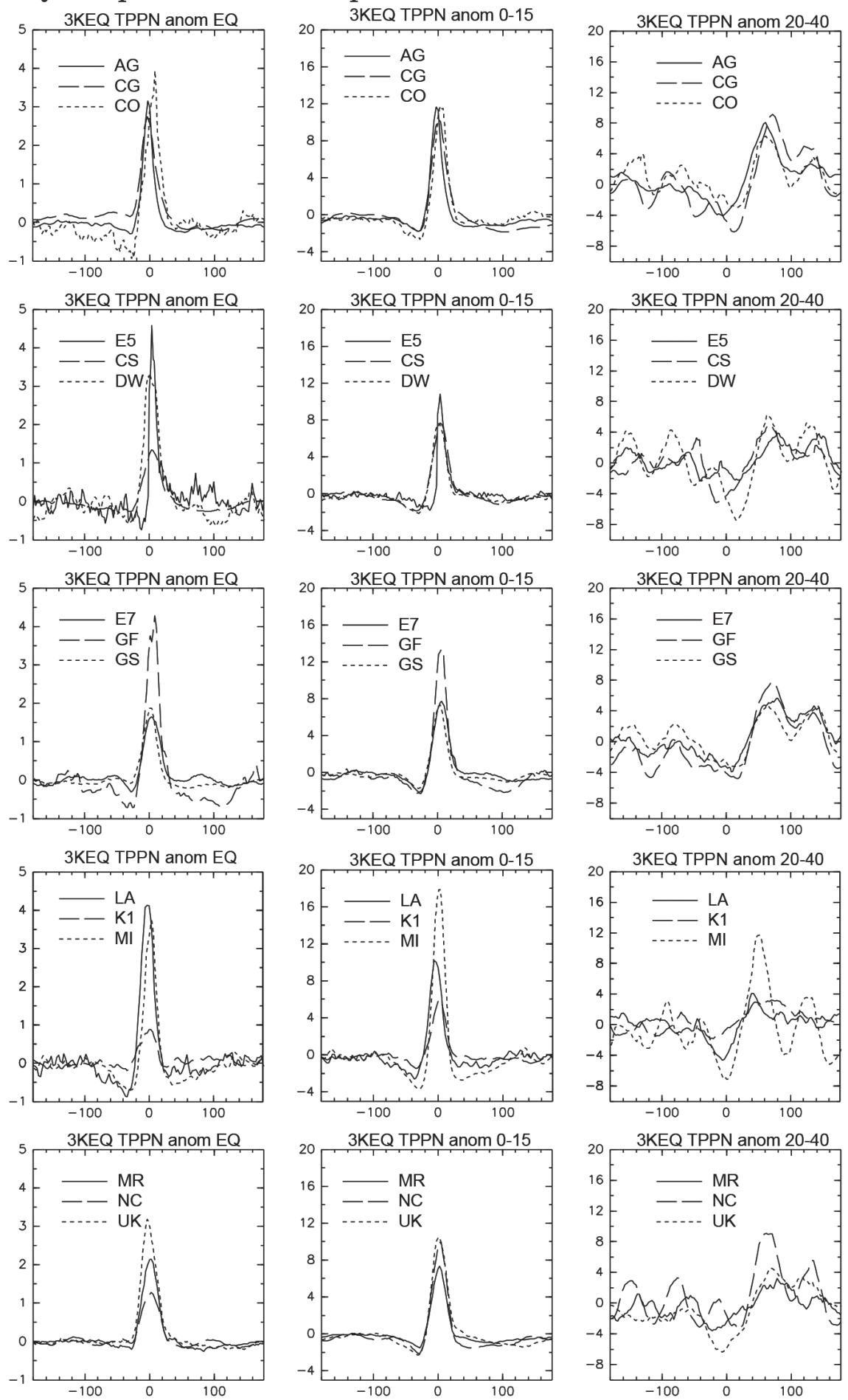

Fig. 5. Zonal distribution of precipitation responses in three selected latitudinal bands of the 3 KEQ runs from the 15 APE models. Left: raw values at the equator (units: $10^{-4} \mathrm{~kg} \mathrm{~m}^{-2} \mathrm{~s}^{-1}$ ). Center: meridional averages over an equatorial band from $-15^{\circ}$ to $15^{\circ}$ (units: $10^{-5} \mathrm{~kg} \mathrm{~m}^{-2} \mathrm{~s}^{-1}$ ). Right: meridional average over a mid-latitude band from $20^{\circ}$ to $40^{\circ}$ (units: $10^{-6} \mathrm{~kg} \mathrm{~m}^{-2} \mathrm{~s}^{-1}$ ). See Table 1 for the label legends. 
Table 2. 3KEQ precipitation responses normalized by CONTROL

\begin{tabular}{lccccccccc}
\hline $\begin{array}{c}\text { model } \\
\text { symbol }\end{array}$ & \multicolumn{2}{c}{ equator $\%$} & \multicolumn{3}{c}{ off-equator $\%$} & \multicolumn{3}{c}{ ave. in 15S-15N \% } & \multicolumn{2}{c}{ ave. in 20N-40N \% } \\
& min & $\max$ & $\min$ & $\max$ & lat. & $\min$ & $\max$ & $\min$ & $\max$ \\
\hline AGU & 79 & 327 & - & - & - & 77 & 261 & 83 & 127 \\
CGAM & 83 & 318 & 58 & 276 & 6 & 70 & 253 & 80 & 131 \\
CSIROstd & 86 & 171 & 59 & 211 & 5 & 75 & 216 & 83 & 119 \\
CSIROold & 66 & 242 & - & - & - & 62 & 261 & 91 & 129 \\
DWD & 82 & 194 & - & - & - & 73 & 206 & 78 & 117 \\
\hline ECMWF05 & 72 & 275 & - & - & - & 78 & 239 & 89 & 115 \\
ECMWF07 & 81 & 198 & 62 & 221 & 5 & 72 & 204 & 89 & 117 \\
GFDL & 70 & 282 & - & - & - & 69 & 281 & 77 & 125 \\
GSFC & 82 & 265 & 51 & 243 & 6 & 69 & 222 & 88 & 113 \\
K1JAPAN & 88 & 156 & 70 & 210 & 5 & 75 & 190 & 94 & 115 \\
\hline LASG & 66 & 258 & - & - & - & 67 & 258 & 75 & 122 \\
MIT & 56 & 333 & 39 & 308 & 5 & 48 & 328 & 74 & 133 \\
MRI & 84 & 238 & 49 & 180 & 5 & 75 & 222 & 82 & 117 \\
NCAR & 85 & 226 & 39 & 287 & 6 & 69 & 244 & 88 & 131 \\
UKMOn96 & 87 & 280 & 37 & 292 & 5 & 68 & 254 & 79 & 122 \\
\hline
\end{tabular}

and the locations of these features differ considerably among the models. For example, the amplitude of the negative geopotential height anomaly at $250 \mathrm{hPa}$, typically centered around $(\lambda, \varphi)=\left(40^{\circ}, \pm 50^{\circ}\right)$ and constituting the propagating Rossby wavetrain ranges from about $30 \mathrm{~m}$ in LASG and MRI to about $100 \mathrm{~m}$ in GFDL and NCAR. The wavenumber 5 feature along the westerly jet is quite prominent in CGAM, DWD, GFDL, MIT, and NCAR, whereas it is almost absent in AGU, CSIROold, K1JAPAN, and LASG.

In the upper troposphere, acceleration of zonal mean zonal wind is observed in some of the models, most notably MIT and NCAR. This acceleration mainly occurs in the upper troposphere above $300 \mathrm{hPa}$, which suggests that it is driven by the Rossby waves excited by the upper tropospheric divergence associated with the precipitation anomaly and emitted to higher latitudes.

It is also evident that appreciable zonal mean responses develop at high latitudes. For ECMWF05 and K1JAPAN, for instance, the high latitudes are covered by intense positive geopotential anomalies at the $850 \mathrm{hPa}$ level (Figs. 8f and 9j). For CSIROold, the northern polar region is occupied by an intense high pressure anomaly that covers the whole troposphere (Figs. 6d and 8d). However, whether or not this is the true time mean response is uncertain, because the zonal mean fields in the high latitudes undergo considerable variations with a fairly long period (exceeding $\sim 100$ d), as described in the ensemble AGCM study on the response to an equatorial SSTA by Nakajima et al.
(2004). Consequently, the zonal mean responses at high latitudes found here may be an artifact that would be smoothed out in a longer averaging interval.

\section{c. Multi-model statistics of the response}

Figure 10a shows the model mean response of geopotential and horizontal wind vectors at $250 \mathrm{hPa}$, and Fig. 10b shows the standard deviation of the geopotential anomalies in $3 \mathrm{KEQ}$ at $250 \mathrm{hPa}$ from the $15 \mathrm{APE}$ models, while Figs. 10c, d show those at $850 \mathrm{hPa}$. The principal features of the dynamical response, which are the Rossby wavetrains originating from the anticyclonic anomalies that develop to the east of the SSTA, the perturbation along the mid-latitude westerly jets at $250 \mathrm{hPa}$, and the zonally dipole geopotential anomalies at the higher latitudes at the $850 \mathrm{hPa}$ level, can be easily identified in the multi-model mean response (Fig. 10a). However, the intensity of these features is generally weaker than in the individual models. For example, the amplitude of the model mean Rossby wavetrain, of about $100 \mathrm{~m}$, is considerably smaller than the representative amplitudes of the Rossby wavetrains in the individual models (Fig. 14a shown later), presumably due to the scattering of the longitudinal phases of the response in the 15 APE models seen in Figs. 6 and 7. In fact, the magnitudes of the standard deviations of the responses is nearly as large as the amplitude of the response at the two levels. The diversity of the mid-latitude response among the models are also reflected in larger standard deviations along the mid-latitude jet (Fig. 10b, d). The large standard deviation at $850 \mathrm{hPa}$ at 
higher latitudes represents the scattering of the geopotential response in the polar region.

It is worth mentioning that the equatorial Kelvin wave response appears a little more clearly in the multi-model mean response than in the individual models (Figs. 6 to 9). The lower-level easterly and upper-level westerly wind anomalies along the equator are easily identifiable to the east of $\varphi \sim 90^{\circ}$. Nearer to the SSTA, the Kelvin response, if it is present to any degree, seems to be completely overshadowed by the intense Rossby responses that develops in the longitudinal regions as is highlighted in the individual models.

\section{d. Vertical structure along the equator}

The vertical structures of the responses along the equator, namely, anomalies of temperature, zonal wind, and vertical p-velocity, are shown for all of the 15 models in Fig. 11. Although the intensity and detailed anomaly patterns are strongly model dependent, very broadly speaking, the overall response structures in all models can be regarded as similar to that described earlier from GFDL, i.e., the warm upward motion develops over the SSTA, the zonal wind anomaly is very different from that of the first baroclinic equatorial Kelvin wave, and the deep warm anomaly is located to the east of, and partially detached from, the SSTA.

The variety in the vertical structure of the temperature anomalies over the SSTA among the models can be related to the varying contributions from the following three components. First, a positive anomaly extending from the surface to about $900 \mathrm{hPa}$ directly caused by the SSTA, second, a negative anomaly around 600 $\mathrm{hPa}$ caused by the melting of frozen hydrometeors, and third, a deep warm anomaly in the upper half of the troposphere. Rather surprisingly, the temperature anomaly in the lowermost troposphere, which is more or less directly controlled by the SSTA of the specified intensity, shows significant diversity; the intensity of the temperature anomaly at $925 \mathrm{hPa}$ varies by a factor of as much as five. This point will be discussed in Section 4.3. Several factors, including the parameterization of physical processes such as surface fluxes, turbulence in the mixed layer, and rain evaporation (or the lack of it), may contribute to this difference.

One would expect the intensities and patterns of the mid-tropospheric cold anomaly and the upper tropospheric warm anomaly to vary among the models because they are directly forced by cloud heating, which is parameterized differently in different models (Table 1). The vertical section of the latent heating anomaly at the equator in the nine models for which the parameterized heating data are available are compared in Fig. 12. Rather surprisingly, the vertical profiles in most of the models exhibit a good amount of similarity; except in DWD and LASG, the heating anomaly is mostly confined to the upper half of the troposphere, although the distribution within the upper troposphere varies among the models. However, it should be noted that the partitioning of the heating anomaly into parameterized and resolved heating is strongly model dependent.

Figures $13 \mathrm{a}$ to $13 \mathrm{i}$ show the vertical distributions of the anomaly of the temperature tendency due to parameterized and resolved cloud processes at the maxima of the precipitation anomaly. In the lower troposphere, the anomalies of parameterized and resolved heating tend to cancel each other out in ECMWF07, GSFC, and NCAR, whereas both components are weak in AGU, ECMWF05, and K1JAPAN. The interpretation of the different contributions from the parameterized and resolved heating in different models is not straightforward because different models employ different cloud schemes. Heating near the surface varies significantly among the models; in ECMWF07, K1JAPAN, and NCAR, shallow but intense cooling exists near the sea surface, which is presumably the effect of the parameterized evaporation of rain.

Comparison between the vertical profiles of heating in these models and the responses at the equator (Fig. 11) reveals that the correspondence between the structure of heating and temperature anomalies above the SSTA is not straightforward. For example, at around $600 \mathrm{hPa}$, a negative temperature anomaly can be found in all models with latent heating data, except for GSFC and LASG, despite the heating anomaly being positive except in AGU. Of course, this is not surprising because other effects, such as advection, diffusion, and other parameterized heating terms, would also affect the heating anomaly. We go no further than pointing out that there is considerable difference in the vertical structure of the response. The most peculiar example is GSFC, where most of the lower troposphere is occupied by a cold anomaly, which reminds us of a similar cold anomaly found at the location of the enhanced precipitation in the composite convectively coupled equatorial waves in the APE CONTROL experiment using the GSFC model (Nakajima et al. 2013). In GSFC, CSIROstd, and K1JAPAN, the vertical velocity anomalies in the lowermost troposphere (below 850 $\mathrm{hPa}$ ) are slightly downward in the convection area at the SSTA. The development of a downward flow anomaly in the convective area may seem counterintuitive. However, considering that basic-state upward motion exists along the equator, and that the anomaly 
3KEQ Anomalies of Geopotential Height, $\mathrm{u}$, and v at $250 \mathrm{hPa}$

(a) AGU

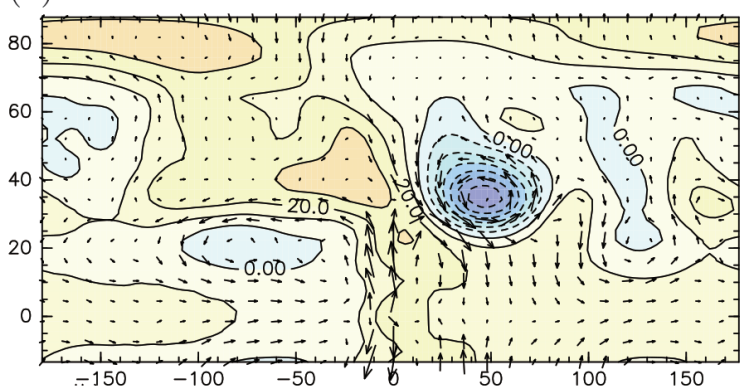

(c) CSIROstd

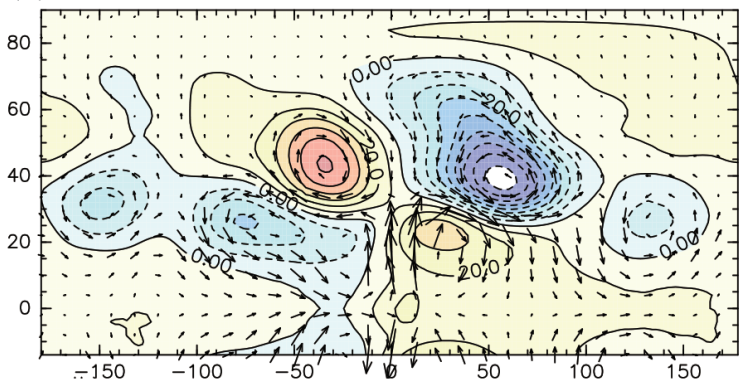

(e) DWD

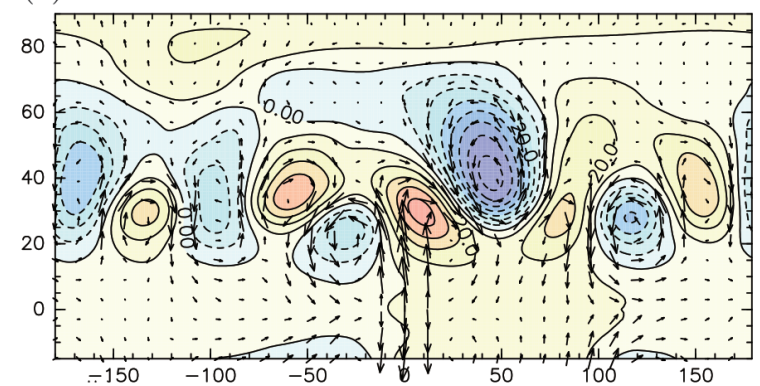

(g) ECMWF07

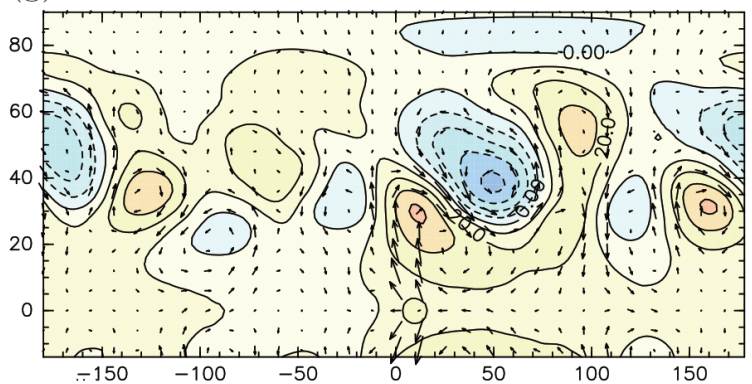

(b) CGAM

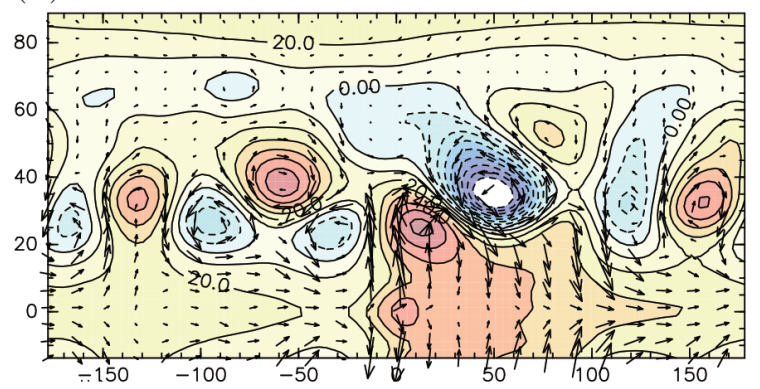

(d) CSIROold

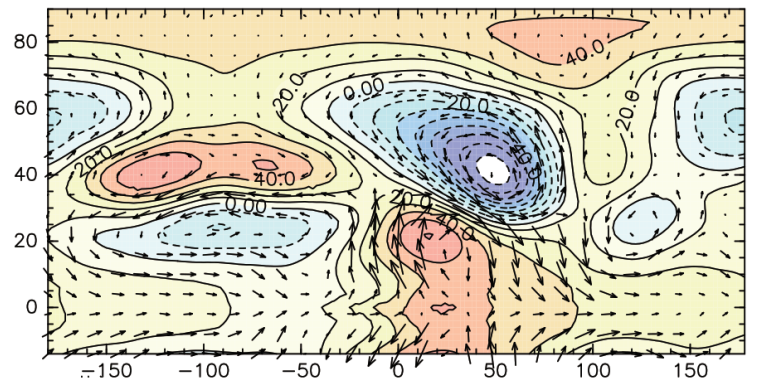

(f) ECMWF05

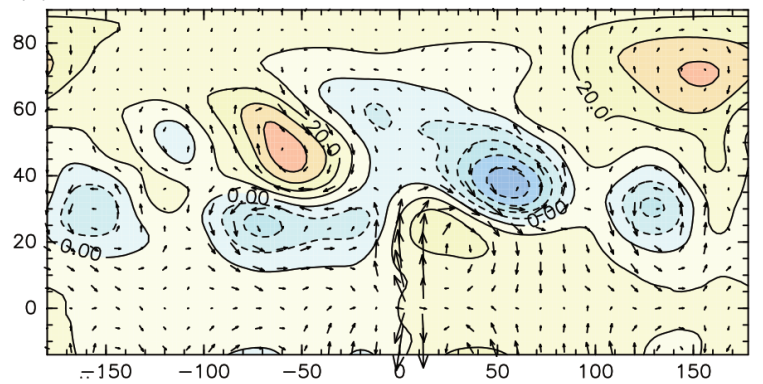

(h) GFDL

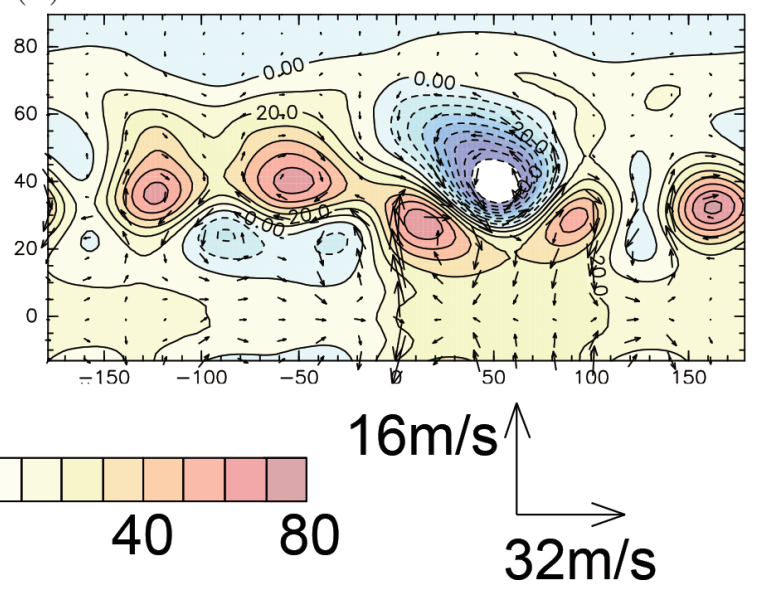

Fig. 6. Time mean anomalies of geopotential height and horizontal velocity vector at $250 \mathrm{hPa}$ in the $3 \mathrm{KEQ}$ runs from the 15 APE models. Contour interval and magnitudes of wind vector components are indicated at the bottom. 
3KEQ Anomalies of Geopotential Height, u, and v at $250 \mathrm{hPa}$ (cont.)

(i) GSFC

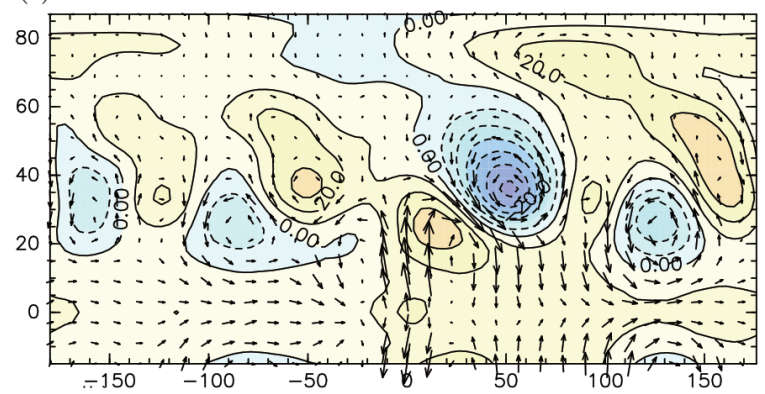

(k) LASG

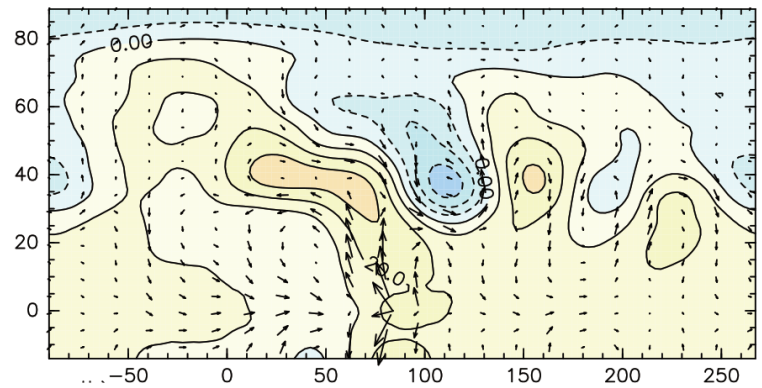

(m) MRI

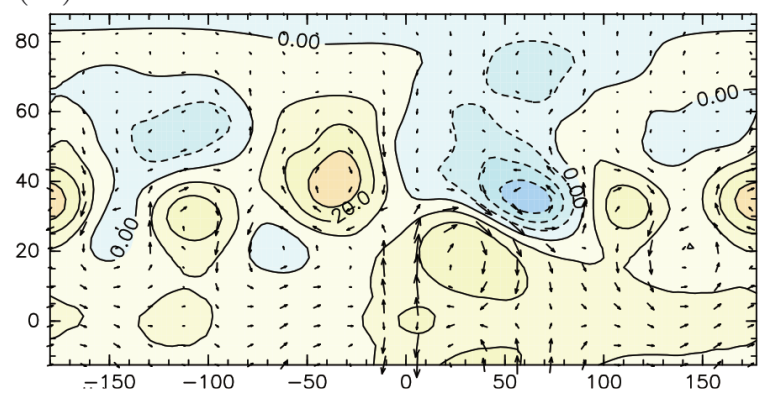

(o) UKMOn96

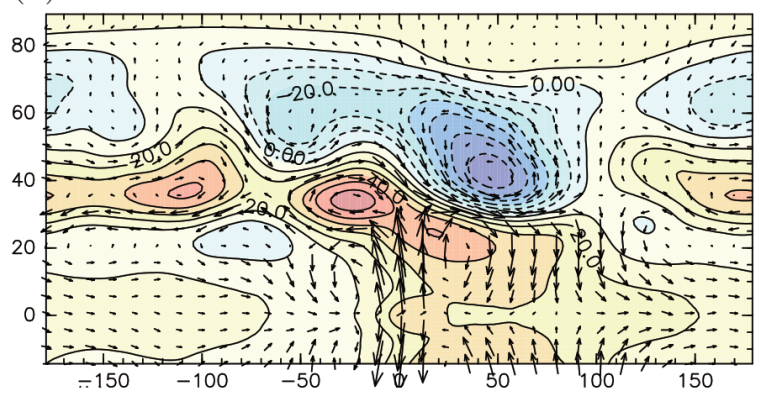

(j) K1JAPAN

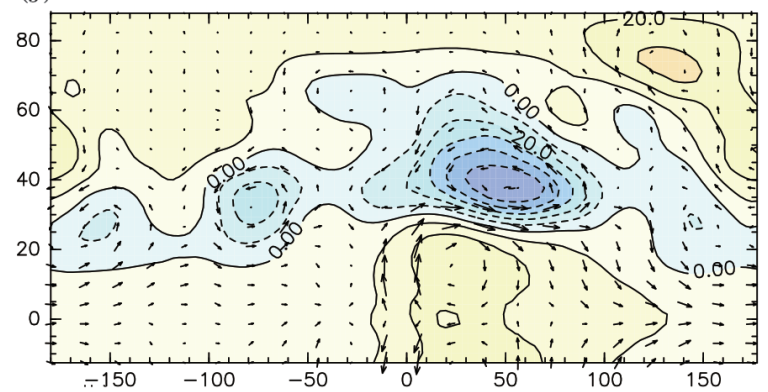

(1) MIT

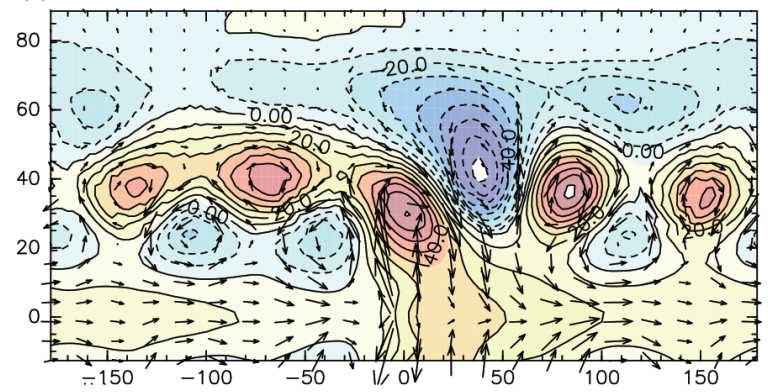

(n) NCAR

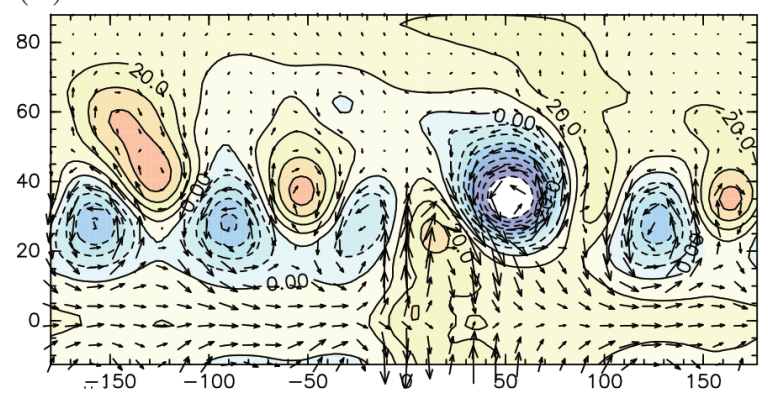

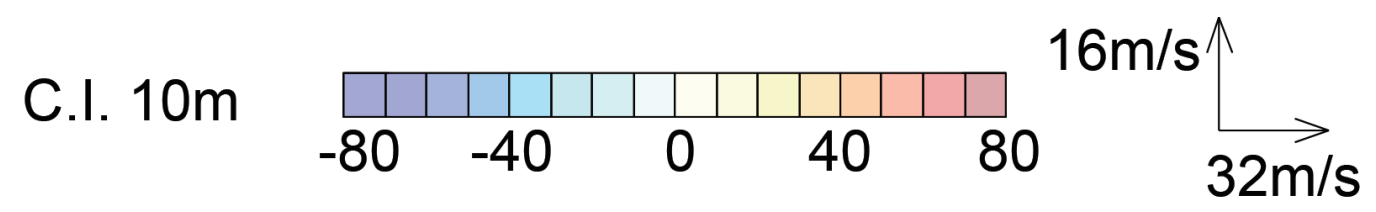

Fig. 7. (Continued from Fig. 6). 
3KEQ Anomalies of Geopotential Height, $\mathrm{u}$, and v at $850 \mathrm{hPa}$

(a) AGU

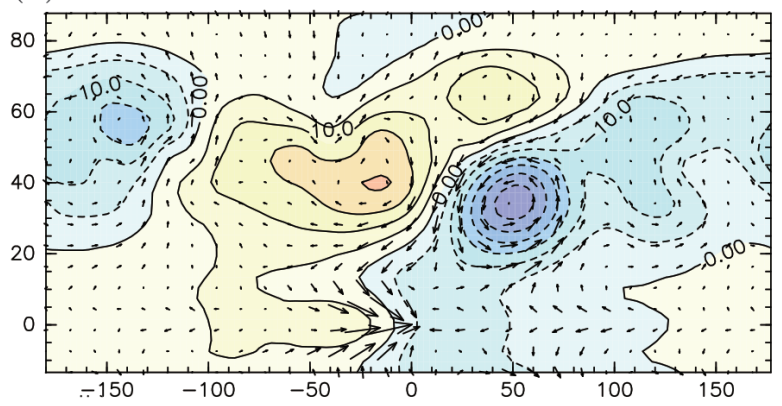

(c) CSIROstd

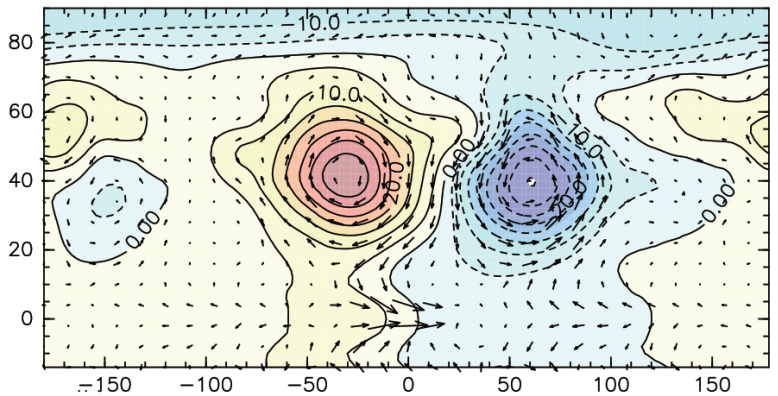

(e) DWD

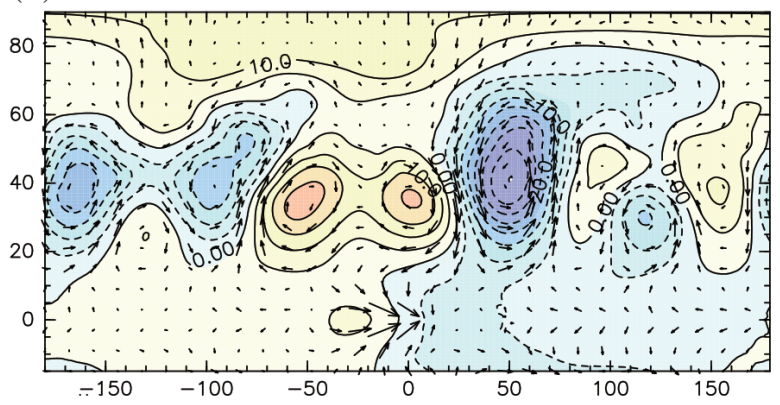

(g) ECMWF07

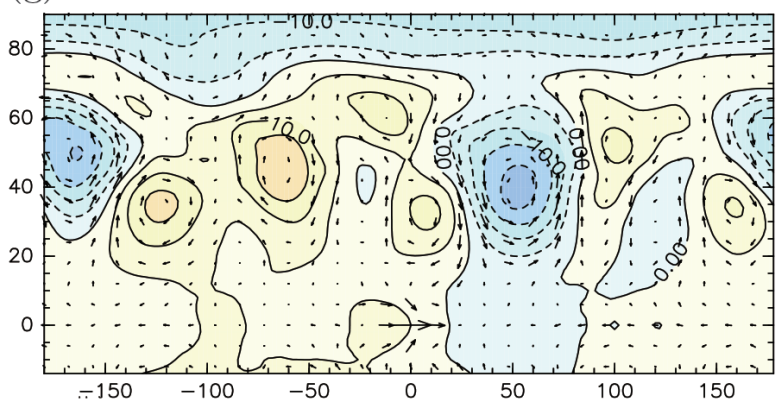

(b) CGAM

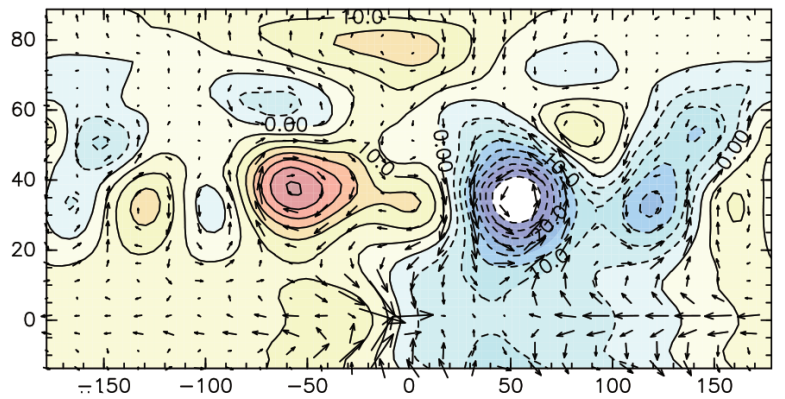

(d) CSIROold

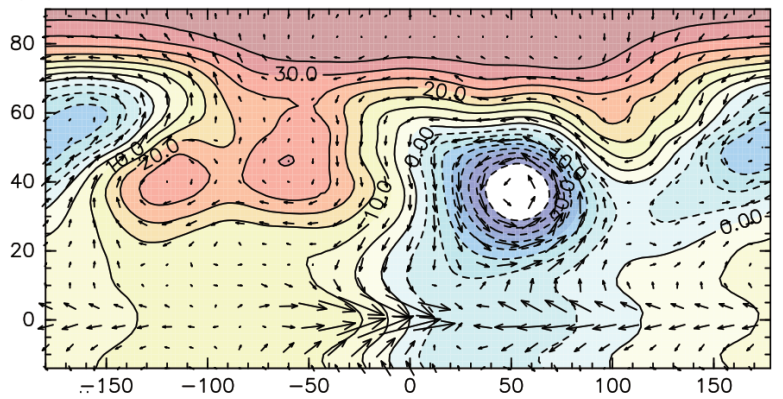

(f) ECMWF05

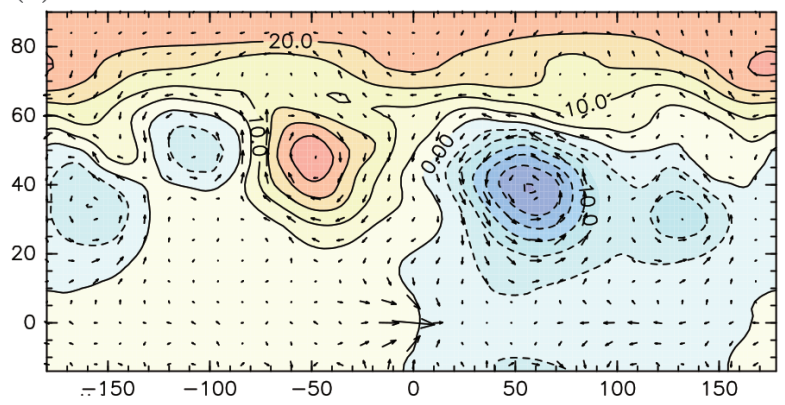

(h) GFDL

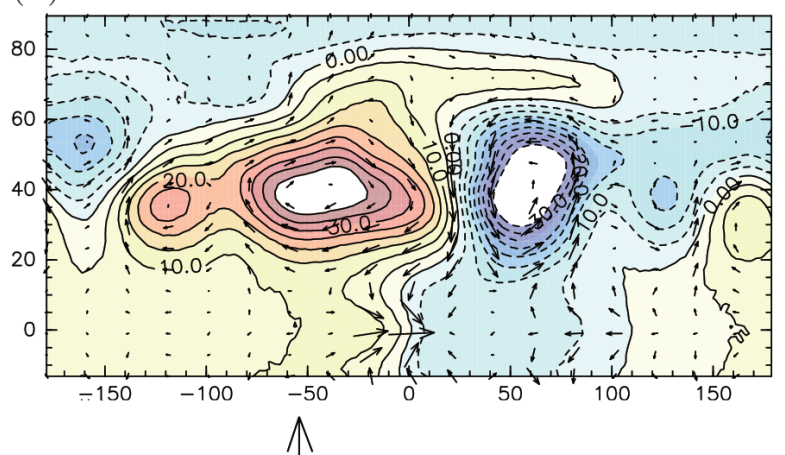

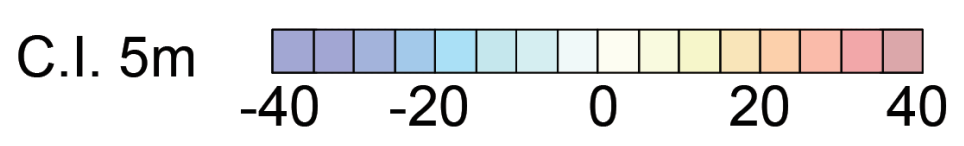

$8 \mathrm{~m} / \mathrm{s}$

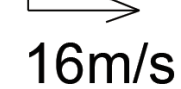

Fig. 8. As Fig. 6 but for $850 \mathrm{hPa}$. 


\section{KEQ Anomalies of Geopotential Height, u, and v at $850 \mathrm{hPa}$} (cont.)

(i) GSFC

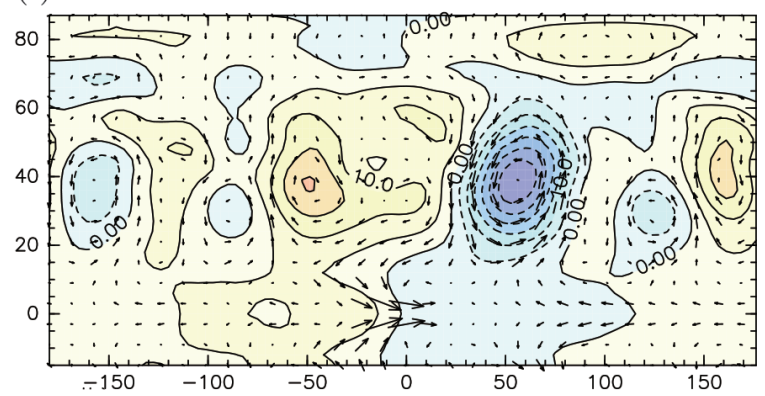

(k) LASG

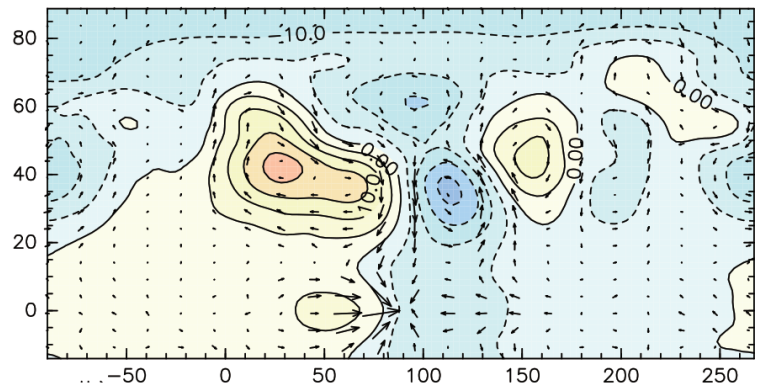

(m) MRI

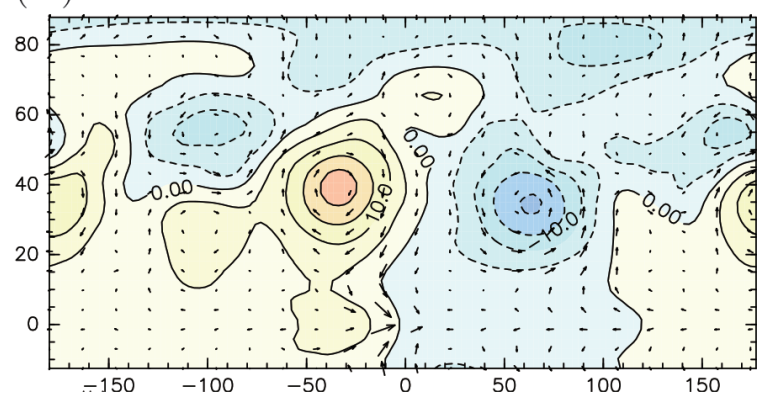

(o) UKMOn96

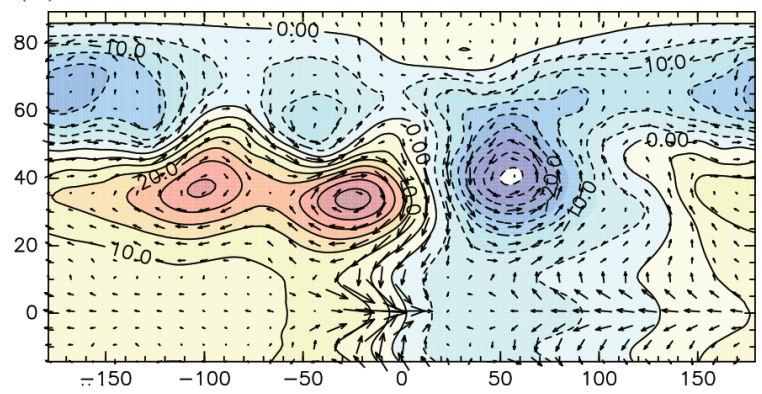

(j) K1JAPAN

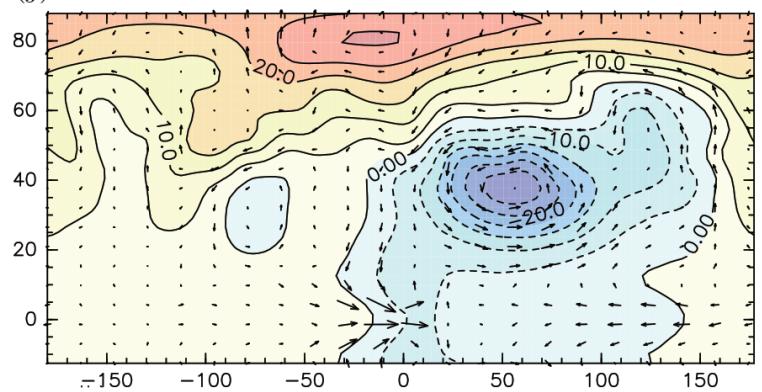

(1) MIT

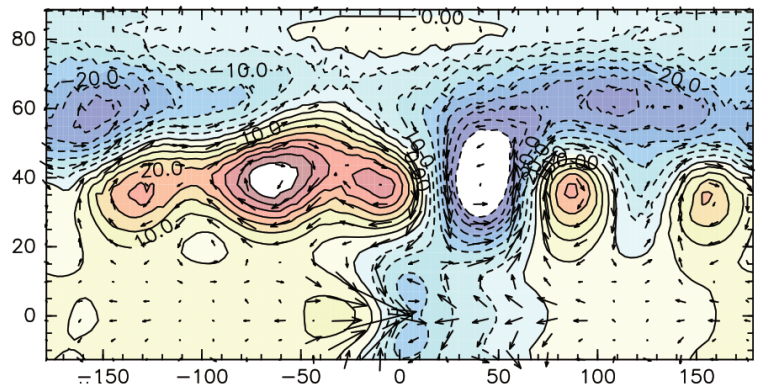

(n) NCAR

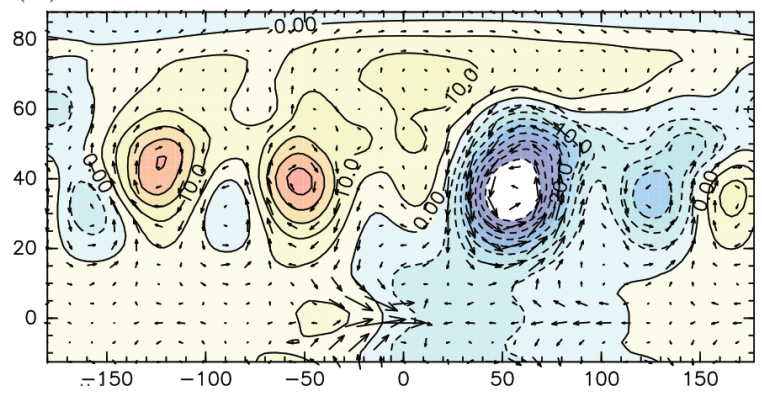

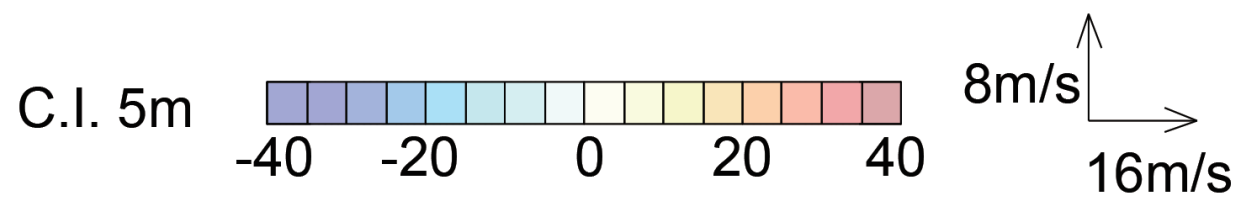

Fig. 9. (Continued from Fig. 8). 


\section{KEQ Multi-Model Statistics of the Response}

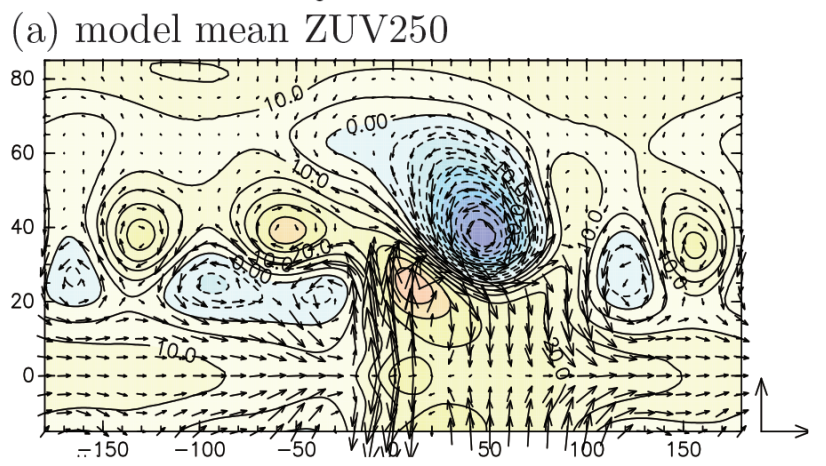

(b) standard deviation Z250

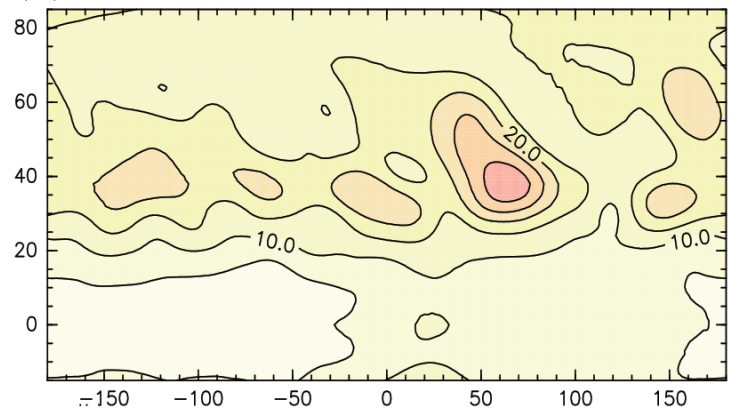

(c) model mean ZUV850

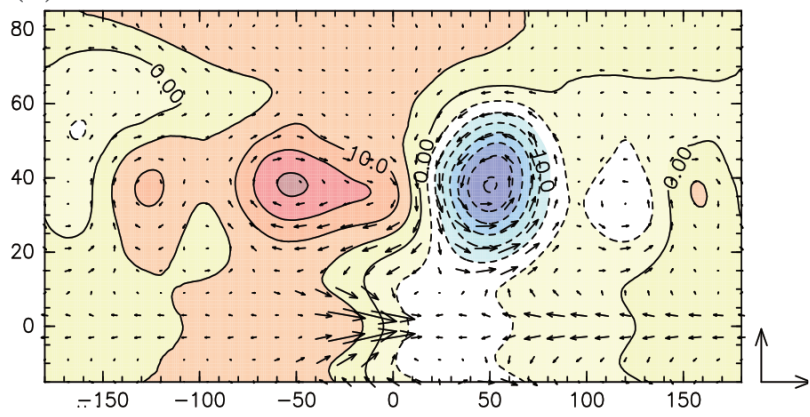

(d) standard deviation Z850

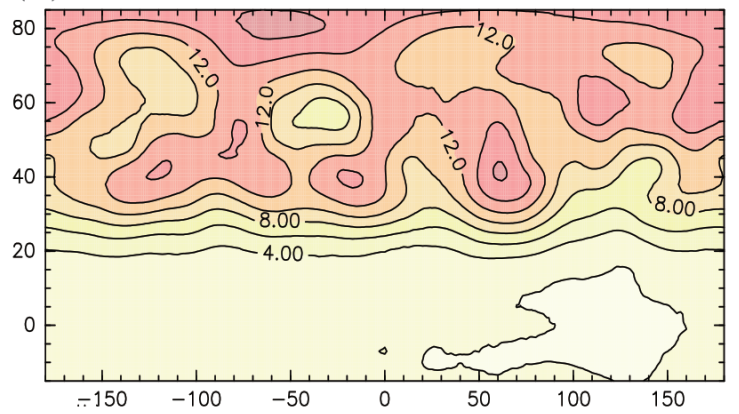

Fig. 10. Time mean anomalies of multi-model statistics of the response in the 3KEQ runs from the 15 APE models. (a) Model averages of the temporal mean anomalies of geopotential height and horizontal velocity vector at $250 \mathrm{hPa}$. Contour interval: $10 \mathrm{~m}$. The unit vectors of zonal and meridional wind are $16 \mathrm{~m} \mathrm{~s}^{-1}$ and $8 \mathrm{~m} \mathrm{~s}^{-1}$, respectively. (b) The standard deviation of the temporal mean anomalies of geopotential height at $250 \mathrm{hPa}$. Contour interval: $5 \mathrm{~m}$. (c) As (a) but for $850 \mathrm{hPa}$. Contour interval: $5 \mathrm{~m}$. The unit vectors of zonal and meridional wind are $8 \mathrm{~m} \mathrm{~s}^{-1}$ and $4 \mathrm{~m}$ $\mathrm{s}^{-1}$, respectively. (d) As (b) but for $850 \mathrm{hPa}$. Contour interval: $2 \mathrm{~m}$.

in convective heating in the lower troposphere above the SSTA is positive at least in two of these models, GSFC and K1JAPAN (Fig. 13e, f), we can expect the development of deep convection even with downward perturbations of vertical velocity.

To the west of the SSTA, the temperature anomaly is generally negative, and seems to be composed of two separate components: one in the lower troposphere between 1000 and $700 \mathrm{hPa}$, and the other in the upper troposphere around $300 \mathrm{hPa}$. These two components seem to develop differently in the models. The low-level negative anomaly tends to be prominent in the models with significant negative precipitation to the west of the SSTA, i.e., AGU, CSIROold, DWD, ECMWF05, ECMWF07, GFDL, LASG, and MIT. In contrast, that in the upper troposphere tends to be prominent in CSIROstd, CSIROold, DWD, GFDL, and UKMOn96, most of which are characterized by a narrow single ITCZ in the CONTROL experiment. The former type of model dependence seems understandable, whereas the latter remains to be considered.

Although certain deep warm anomalies are commonly evident around several thousand kilometers to the east of the SSTA in all of the models, their longitudinal and vertical distributions, and their intensity, vary considerably among the models. Comparison among the equatorial sections suggests that the structure of this warm anomaly seems to be related to the structure of the temperature anomaly over the SSTA to some extent. For example, in ECMWF05, ECMWF07, and GSFC, where the mid-tropospheric cooling over the SSTA is significant (Figs. 11f, g, i), the detached warm anomalies are vertically shallow. The intensity of the detached warm anomaly seems to correlate with the intensity of the Rossby wavetrains generated from the SSTA in mid-latitudes. For example, the models that exhibit strong detached anomalies, namely, CGAM, CSIROold, MIT, and UKMOn96, are charac- 
3KEQ Anomalies of $\mathbf{T}, \mathbf{u}$, and $\omega$ at the Equator

(a) AGU

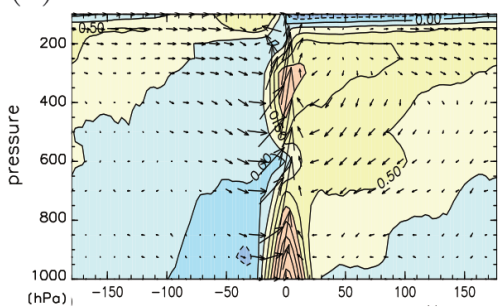

(d) CSIROold

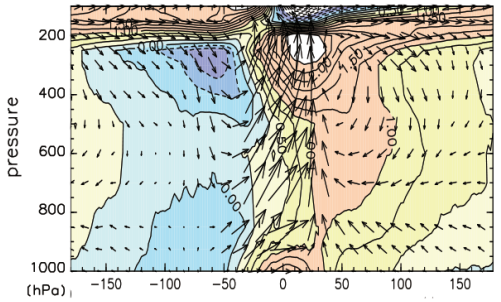

(g) ECMWF07

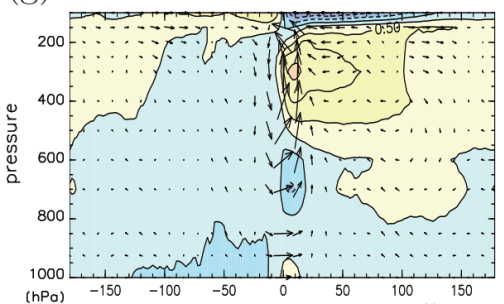

(j) K1JAPAN

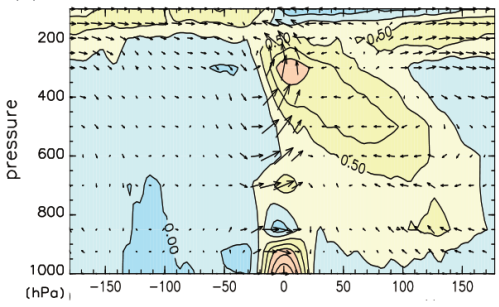

(m) MRI

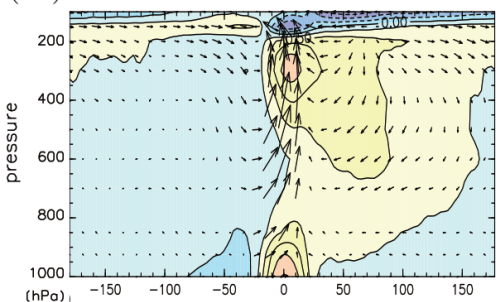

(b) CGAM

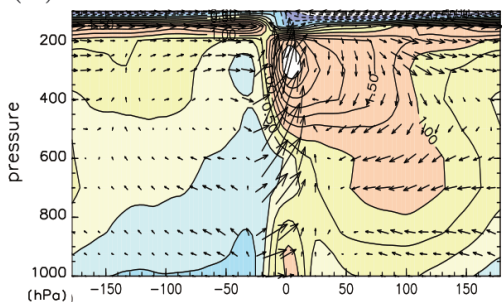

(e) DWD

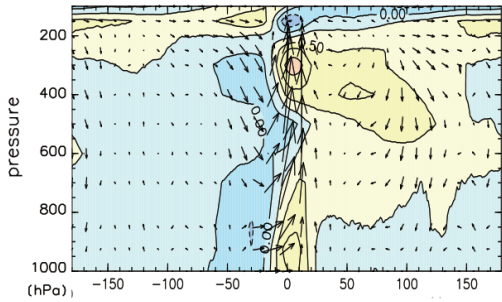

(h) GFDL

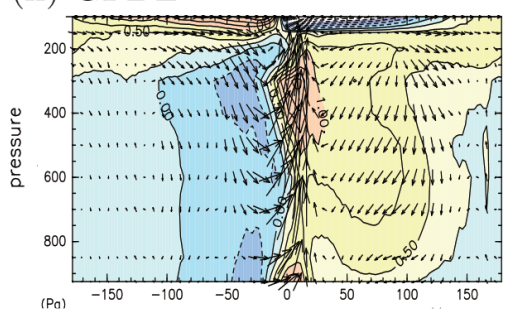

(k) LASG

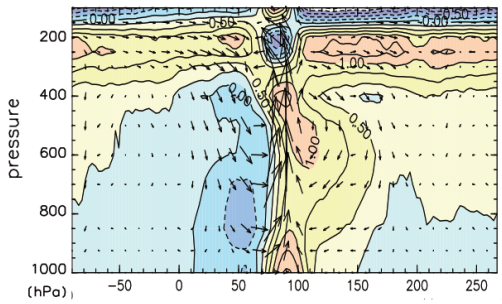

(n) NCAR

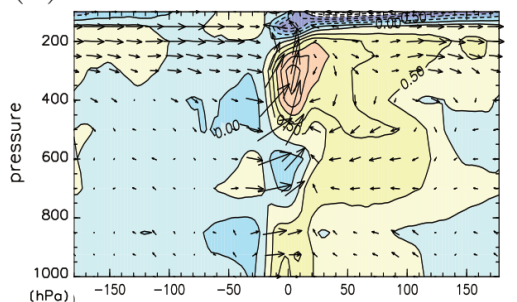

(c) CSIROstd

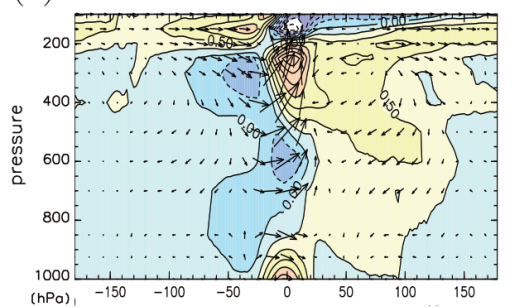

(f) ECMWF05

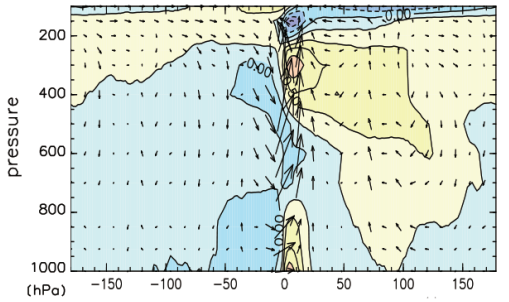

(i) GSFC

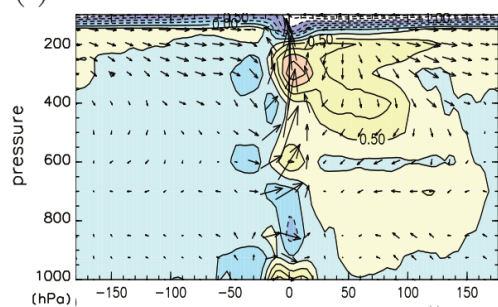

(1) MIT

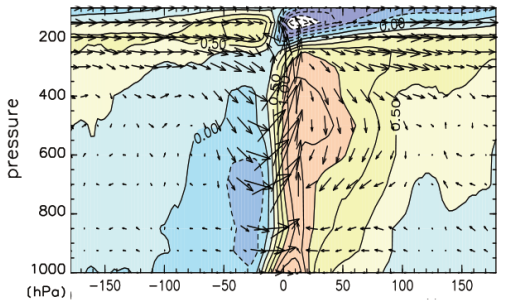

(o) UKMOn96

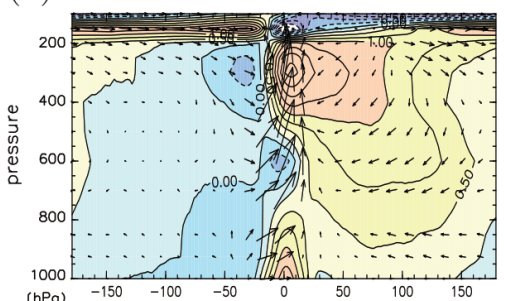

$-0.3 \mathrm{~Pa} / \mathrm{s}$

\section{C.I. $0.25 \mathrm{~K}$}

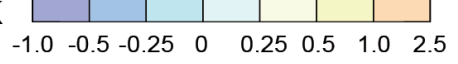

$20 \mathrm{~m} / \mathrm{s}$

Fig. 11. Vertical distributions of time mean anomalies of temperature, zonal velocity, and p-velocity along the equator in the 3KEQ runs from the $15 \mathrm{APE}$ models. Contour interval and magnitudes of wind vector components are indicated at the bottom. 
3KEQ Anomalies of T Tendency by Cloud at the Equator

(a) AGU ci: $8 \mathrm{E}-5 \mathrm{~K} \mathrm{~s}^{-1}$

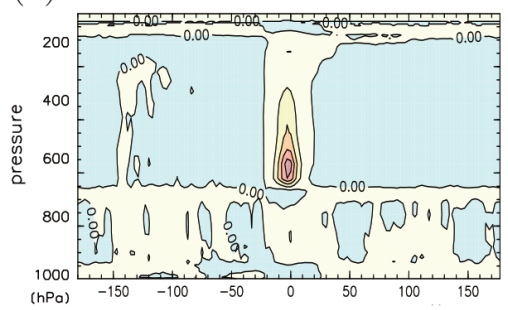

(d) ECMWF07 ci:2E-5 $\mathrm{K} \mathrm{s}^{-1}$

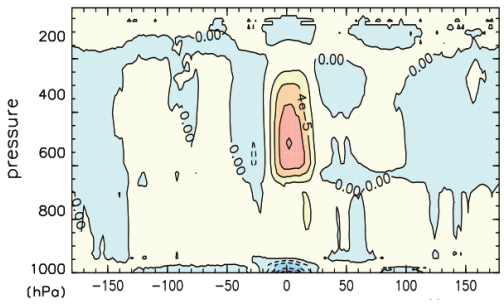

(g) LASG ci:4E-5 $\mathrm{K} \mathrm{s}^{-1}$

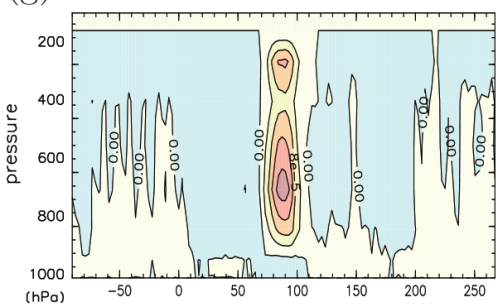

(b) DWD ci:4E-5 $\mathrm{K} \mathrm{s}^{-1}$

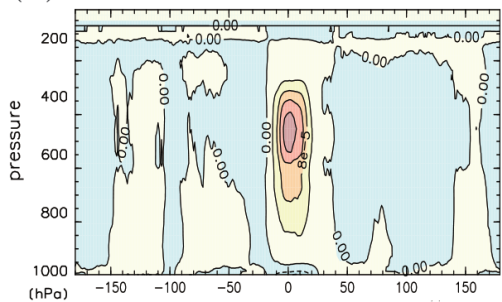

(e) GSFC ci:4E-5 $\mathrm{K} \mathrm{s}^{-1}$

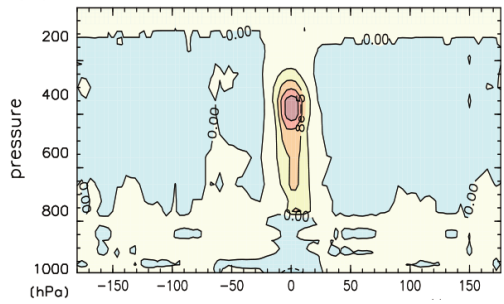

(h) NCAR ci:2E-5 $\mathrm{K} \mathrm{s}^{-1}$

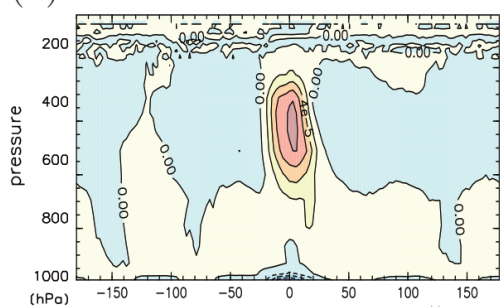

(c) ECMWF05 ci:4E-5 $\mathrm{K} \mathrm{s}^{-1}$

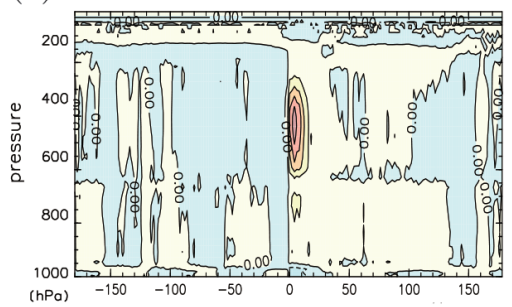

(f) K1JAPAN ci:1E-5 $\mathrm{K} \mathrm{s}^{-1}$

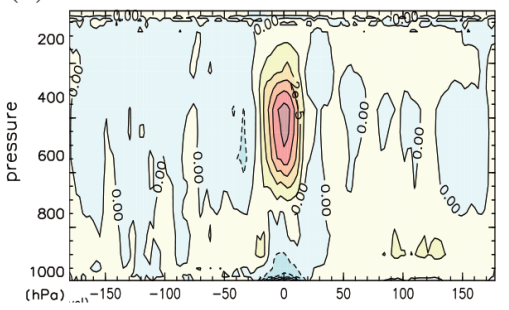

(i) UKMO ci:4E-5 $\mathrm{K} \mathrm{s}^{-1}$

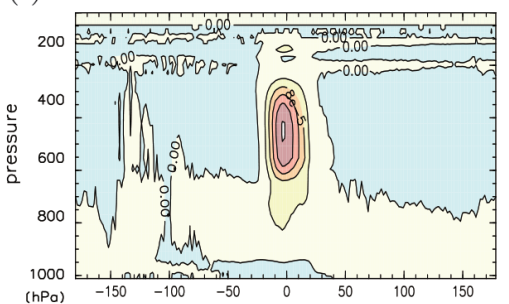

Fig. 12. Vertical distributions of time mean temperature tendencies at the equator in the 3 KEQ runs from the 15 APE models. The sum of parameterized and resolved tendensies are plotted. Contour interval is indicated at the top of each panel. Vertical axis is pressure. Units: $\mathrm{K} \mathrm{s}^{-1}$.

terized by intense Rossby wavetrains (Figs. 6bc and 7lo). Nevertheless, the correspondence is not perfect; for example, in NCAR, the Rossby wavetrain is prominent (Fig. 7n), but the detached warm anomaly is not particularly conspicuous (Fig. 11n). Other factors, such as the structure of the zonal mean zonal wind, and the vertical structure of heating over the SSTA, may also be important. As the heating anomalies at the corresponding locations are quite weak (Figs. 12a to 12i), it is probable that these temperature anomalies have a dynamical origin. Further analysis is required to clarify the mechanism that drives the generation and maintenance of the detached warm anomaly.

\subsection{Relationships among the variables}

In the preceding subsections, we have described variety in the atmospheric response to the 3KEQ SSTA in different models and examined several different variables separately. In this subsection, we examine the relationships among the responses of different variables derived from the 3KEQ runs, and try to identify the causes of the variability identified so far in the comparisons among the APE models.

\section{a. Dynamical response}

In the previous subsection, we pointed out large varieties in the equatorial precipitation responses (Figs. 4 and 5; Table 2) and extratropical geopotential responses in the upper (Figs. 6 and 7) and lower troposphere (Figs. 8 and 9). Here, we examine the relationship between the intensity of the tropical precipitation anomaly and the extratropical response.

Figure 14a shows the relationship between the amplitude of the precipitation anomaly averaged over the $\pm 15^{\circ}$ latitude band, and the amplitude of the geopotential height anomaly at $250 \mathrm{hPa}$. Here, the amplitude of the geopotential height anomaly is calculated as the difference between the maximum and minimum values 


\section{KEQ Anomalies of T Tendency over the SSTA}

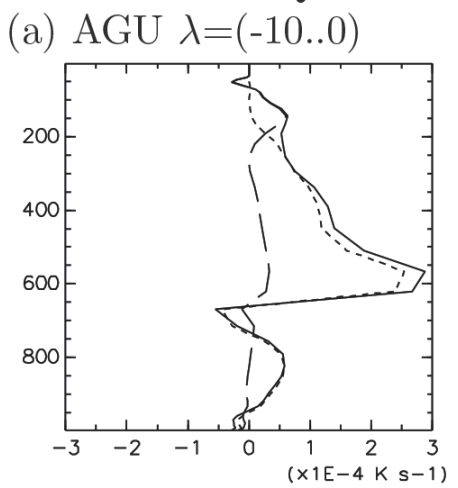

(d) ECMWF07 $\lambda=(0 . .10)$

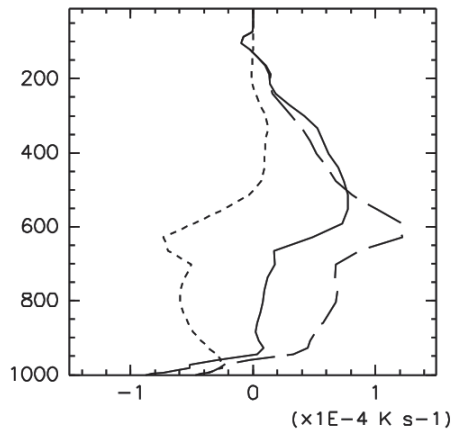

(g) LASG $\lambda=(0 . .10)$

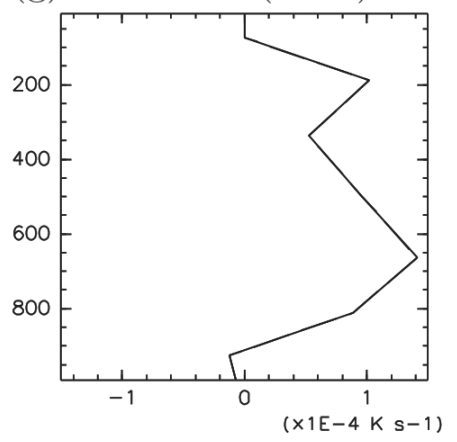

(b) DWD $\lambda=(0 . .10)$

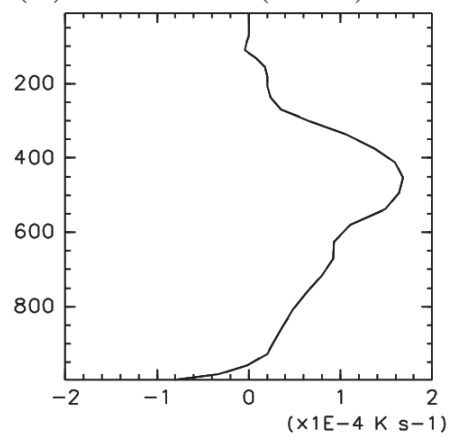

(e) GSFC $\lambda=(0 . .10)$

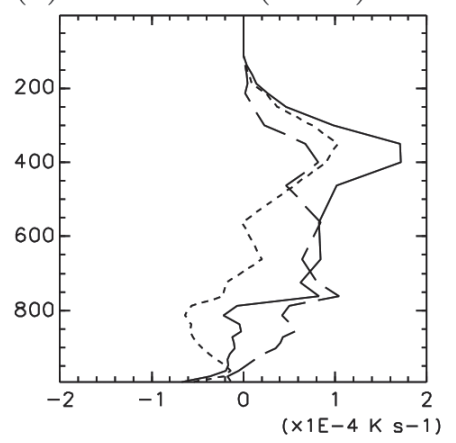

(h) NCAR $\lambda=(0 . .10)$

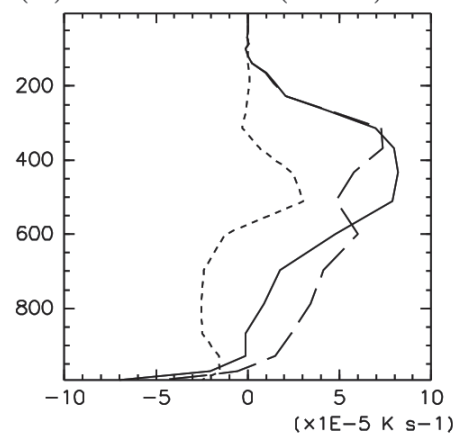

(c)ECMWF05 $\lambda=(0 . .10)$

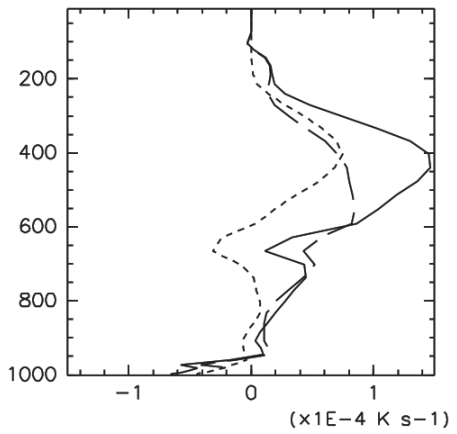

(f) K1JAPAN $\lambda=(0 . .10)$

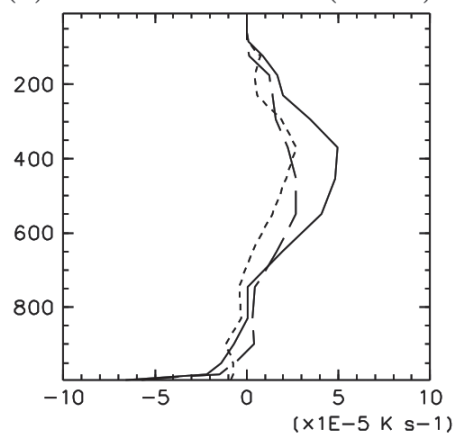

(i) UKMO $\lambda=(0 . .10)$

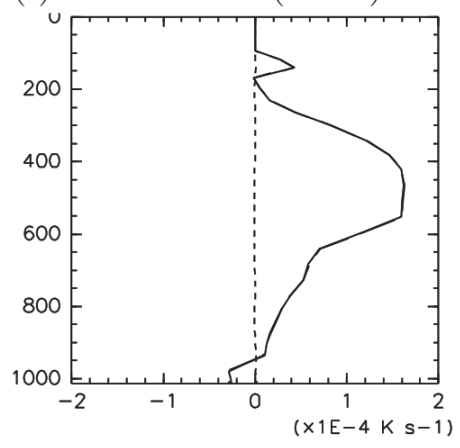

Fig. 13. Vertical profiles of time mean temperature tendencies over the SST anomaly in the 3KEQ runs from the nine APE models for which data were available. Units: $\mathrm{K} \mathrm{s}^{-1}$. Vertical axis is pressure. Dotted and dashed lines indicate tendencies due to resolved clouds and parameterized convection, respectively, while solid line indicate the sum of the two. Note that heating due to resolved clouds is not available for DWD and LASG.

of the eddy component geopotential height, practically showing the intensity of the Rossby wavetrains at 250 $\mathrm{hPa}$. The amplitude of precipitation is represented in units of the equivalent amount of latent heat: $1000 \mathrm{~W}$ $\mathrm{m}^{-2}$ corresponds to $4 \times 10^{-4} \mathrm{~kg} \mathrm{~s}^{-1} \mathrm{~m}^{-2}$. We see that both of the amplitudes vary by a factor of 2.5 , and seem to be in proportion to each other. A similar correlation can also be observed for the wave amplitude in the lower troposphere (not shown). This correlation suggests that the variability in the amplitude of extratropical waves is mainly due to the varying intensity of the tropical precipitation anomaly. Nevertheless, a considerable deviation from this correlation remains; for example, despite the precipitation amplitudes of MRI and CSIROstd being almost the same, the amplitudes of the extratropical waves in these two models differ 
(a)

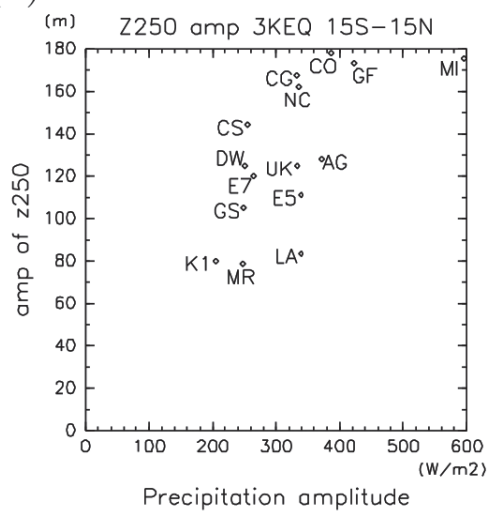

(b)

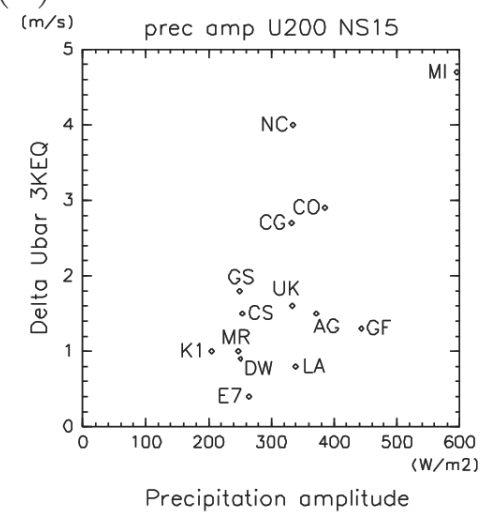

(c)

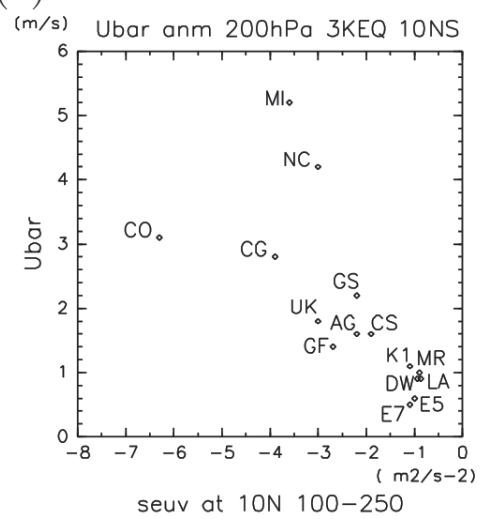

Fig. 14. Scatter plots of the dynamical response of the variables in the 3KEQ runs from the 15 APE models. (a) The peak-to peak amplitudes of the precipitation anomaly vs that of the geopotential height anomaly at $250 \mathrm{hPa}$. (b) The peak-to-peak amplitudes of the precipitation anomaly vs the zonal mean wind anomaly at $200 \mathrm{hPa}$. Plotted values are those averaged over the equatorial latitudinal band within $\pm 15^{\circ}$. (c) Poleward zonal momentum flux associated with the stationary eddy at $10^{\circ} \mathrm{N}$ averaged for pressure levels between 100 and $250 \mathrm{hPa}$ vs the zonal mean acceleration averaged within $10^{\circ}$ of the equator at $200 \mathrm{hPa}$. See Table 1 for the label legends.

by a factor of almost two. Several other issues, such as the vertical structure of heating, and the structure of the mean flow, should also be considered, although we do not go into these issues any further in this paper.

Figure 14b shows the relationship between the amplitude of the precipitation response averaged within the $\pm 15^{\circ}$ latitude band, and the amplitude of the zonal mean zonal wind anomaly at $200 \mathrm{hPa}$ within the same region. Although a vaguely positive correlation may be present, the varieties within the zonal mean wind response are quite large. Figure $14 \mathrm{c}$ shows the relationship between the upper tropospheric meridional transport of zonal momentum by the stationary eddy at $10^{\circ} \mathrm{N}$, and the amplitude of the zonal mean zonal wind anomaly at $200 \mathrm{hPa}$ averaged within the $\pm 10^{\circ}$ latitude zone. The more conspicuous correlation between the two variables found in the figure indicates the important role of the stationary eddy in the zonal mean wind acceleration. However, examination of the time series data from the APE runs shows that the zonal mean zonal winds fluctuate over $\mathrm{O}(1) \mathrm{m} \mathrm{s}^{-1}$ and with time scales ranging from $\mathrm{O}(10)-\mathrm{O}(100) \mathrm{d}$ in each model, so that the precise degree of zonal wind acceleration is uncertain.

\section{b. Factors controlling the precipitation anomaly}

We now examine the impact of several variables on the response of precipitation by comparing the amplitude of the precipitation anomaly with: 1) the amplitude of the low-level temperature anomaly, which influences the degree of convective instability; 2) the zonal mean intensity of precipitation in the CONTROL experiment, which represents the "basic state" of precipitation; and 3) the amplitude of the evaporation anomaly, which supplies the moisture for the enhanced precipitation. We use the following two values for the amplitude of the anomalies: one is the intensity averaged within $\pm 5^{\circ}$ of the equator, which reflects the variety of the meridional structure among the models, and the other is the intensity averaged within $\pm 15^{\circ}$ of the equator, which indicates the longitudinal variation of the precipitation anomaly over the ITCZ as a whole. It should be noted that the SSTA in ECMWF05 lacks its western half, so that the real response of this model is presumably stronger than is shown here.

Figure 15a is a scatter plot showing the amplitude of the precipitation anomaly versus the amplitude of the temperature anomaly at $925 \mathrm{hPa}$, and averaged within the $\pm 5^{\circ}$ latitude band. It is rather surprising that the temperature amplitude varies by a factor of four among the models. A vague positive correlation can be seen between the two variables, but it is far from conclusive. Figure $15 \mathrm{~d}$ is a similar scatter plot, but for the average within the $\pm 15^{\circ}$ latitude band. The variation among the models is smaller than in Fig. 15a, although the temperature amplitude still varies by a factor of three, and the two variables seem to be positively correlated. These comparisons suggest that the intensity of the precipitation response to the localized equatorial SSTA is controlled to some extent by 
(a)

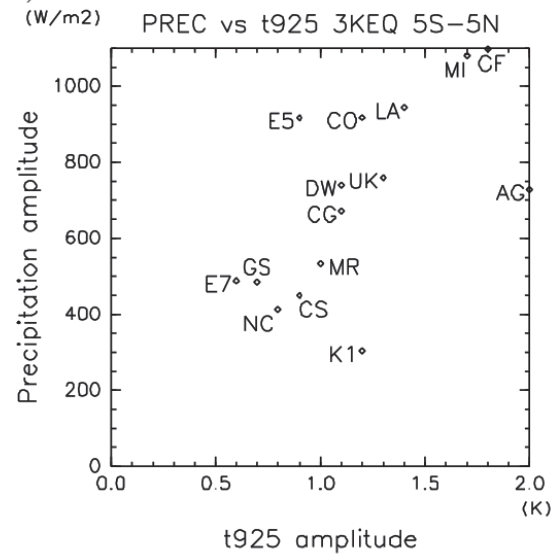

(b)

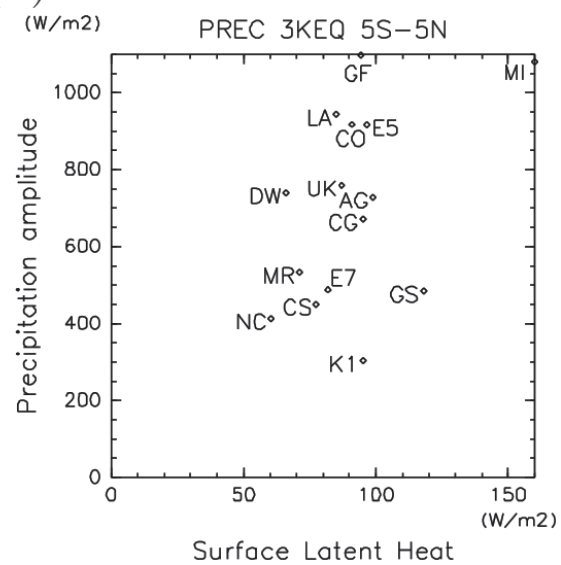

(c)

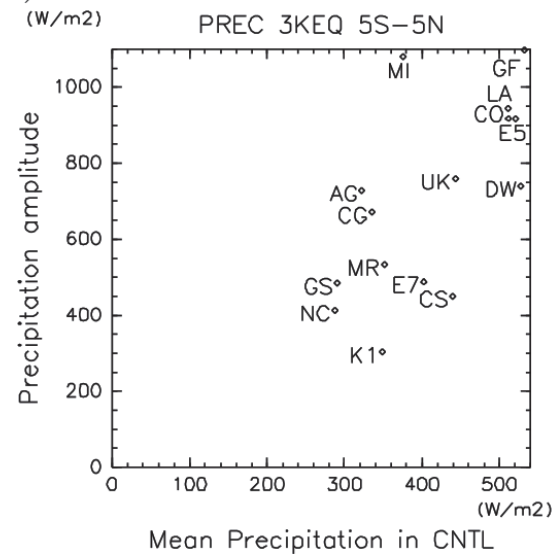

$(\mathrm{d})$

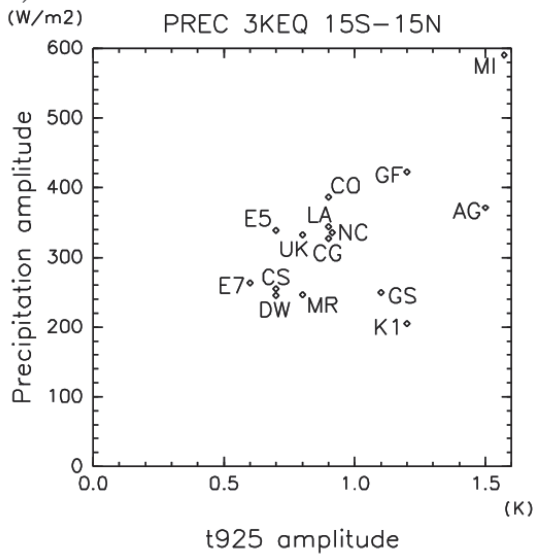

(e)

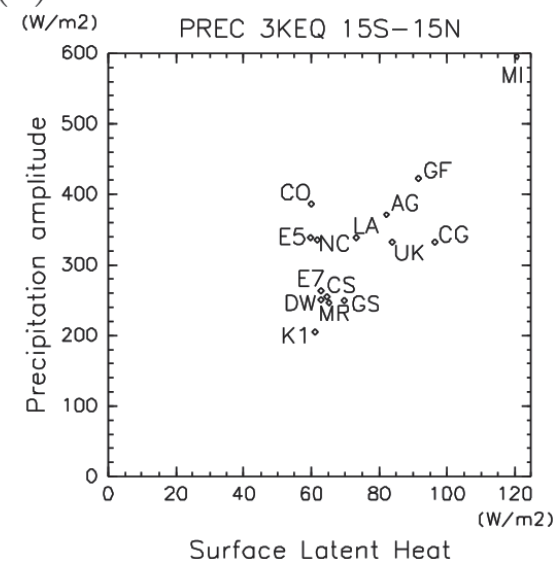

(f)

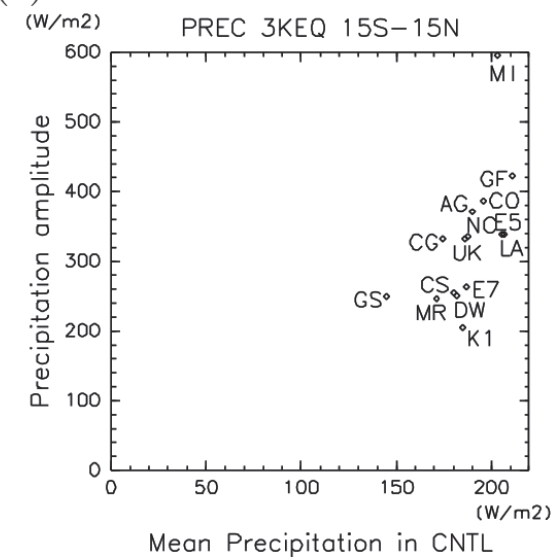

Fig. 15. Scatter plots of the peak-to-peak amplitudes of the precipitation anomalies and of the anomalies of several variables in the 3KEQ runs from the 15 APE models. (a) Temperature anomaly at $925 \mathrm{hPa}$ versus the precipitation anomaly, both of which are averaged within a $\pm 5^{\circ}$ latitude band around the equator. (b) As (a) but for the latent heat flux anomaly. (c) As (a) but for the precipitation intensity in CONTROL. (d) As (a) but for the averages within $\pm 15^{\circ}$ of the equator. (e) As (b) but for the averages within $\pm 15^{\circ}$ of the equator. (f) As (c) but for the averages within $\pm 15^{\circ}$ of the equator. See Table 1 for the label legends. 
the processes that govern the low-level temperature response to the SSTA.

Figure $15 b$ is a scatter plot showing the amplitude of the precipitation anomaly versus the amplitude of the surface latent heat flux anomaly averaged within the $\pm 5^{\circ}$ latitude band. The amplitude of the latent heat flux anomaly varies by a factor of three, and the correlation between the two amplitudes is very weak. Figure 15e is a similar scatter plot, but for the average within the $\pm 15^{\circ}$ latitude band. The scattering of the amplitude of the latent heat flux response is narrower than that in the $\pm 5^{\circ}$ latitude band, and varies by a factor of two. While there seems to be good correlation between the two amplitudes, this relationship is heavily influenced by the existence of an isolated point, MIT, without which the other data points are only clustered around the average. Even if we admit the proportionality between the amplitudes of the precipitation and the latent heat flux anomalies, it should be remarked that the latent heat amplitude is only about a quarter of the precipitation amplitude. Further major factors remain that also contribute to the intensity of precipitation anomaly.

Figure $15 \mathrm{c}$ plots the amplitude of the precipitation anomaly versus the zonally averaged precipitation in the CONTROL experiment averaged within the $\pm 5^{\circ}$ latitude band. There seems to be a positive correlation in this figure, which may be considered reasonable, because, as was pointed out earlier in this section (Fig. 4 ), the precipitation response near the equator in the 3KEQ run of a particular model depends heavily on the tropical structure of precipitation and the circulation of the corresponding CONTROL run. Figure $15 \mathrm{f}$ is a similar scatter plot, but for the average within the $\pm 15^{\circ}$ latitude band. Scattering among the models becomes smaller, since the meridional change across the ITCZ is averaged out. A weak positive correlation is noted, suggesting that the meridionally averaged precipitation response to a localized equatorial SSTA is stronger in models where the ITCZ precipitation is large.

In summary, we have suggested the existence of positive correlations between some pairs of variables, but, overall, the correlations are not conclusive. We have also presented cases where clear correlations are not seen. However, we consider that it is worthwhile to present even such seemingly negative results, because they provide additional information regarding the characteristics of the variations realized in the participating models. In fact, it should be stressed that even the temperature response to the SSTA at low levels, which is expected to be rather trivial, scatters across a wide range. The mechanism underlying this scattering should be pursued, but we leave this for future studies.

\subsection{Linearity of response to localized SST anomaly}

By comparing the results of the 3KEQ and 1KEQ experiments, we can obtain some idea of the extent of the linearity of the response to the equatorial localized SSTA. The overall structure of the responses to the 1KEQ SSTA, whose distributions are not shown here, are mostly the same as those to the 3KEQ SSTA, except that they are considerably weaker as described below.

Figure 16a shows the scatter plot of the amplitudes of precipitation anomalies for $1 \mathrm{KEQ}$ and $3 \mathrm{KEQ}$ within the $\pm 5^{\circ}$ latitude band. Figure $16 \mathrm{~b}$ shows a similar scatter plot, but for the averages within the $\pm 15^{\circ}$ latitude band. UKMOn96 is not plotted because the 1 KEQ experiment was not performed in this model. For a linear response, the data points should distribute along a line with a slope of 3 . In fact, the behavior of many of the models follows the expected relationship in both figures.

Figure 16c shows the scatter plot of the amplitudes of extratropical geopotential anomalies at $250 \mathrm{hPa}$ in $1 \mathrm{KEQ}$ and $3 \mathrm{KEQ}$. Although the amplitudes in $3 \mathrm{KEQ}$ and $1 \mathrm{KEQ}$ are certainly positively correlated, the ratio between the amplitude in 3KEQ and that in $1 \mathrm{KEQ}$ is far less than 3 , which may indicate the presence of some nonlinearity that suppresses extratropical wave amplitudes. However, we should remember the possible contributions from the background fluctuations that exist regardless of the SSTA. In fact, the amplitudes of quasi-stationary waves in the CONTROL experiment are as large as 40 to $70 \mathrm{~m}$ depending on the model. If we tentatively set the background level to be $40 \mathrm{~m}$ in both experiments, and draw a line with a slope of 3 originating from this background level, the results from the models seems to be well explained. Quantitative examination of this point requires more careful statistical consideration, and is left for future research.

\section{Response to wavenumber one variation of SST: $3 \mathrm{KW} 1$}

\subsection{Characteristic features of response}

In this section, the 15 AGCM runs with 3KW1 SSTA are presented and compared with one another. As were done for $3 \mathrm{KEQ}$, before the full comparison and subsequent presentation of the multi-model statistics, we will identify the principal features of the response to the 3KW1 SSTA by focusing on one of the models, which in this case will be NCAR, as a clear example in this subsection. As the intensity of the response is quite strong, we choose to describe the time mean response of NCAR to the 3KW1 SSTA in Fig. 17 by showing mainly the raw values of variables rather than 
(a)

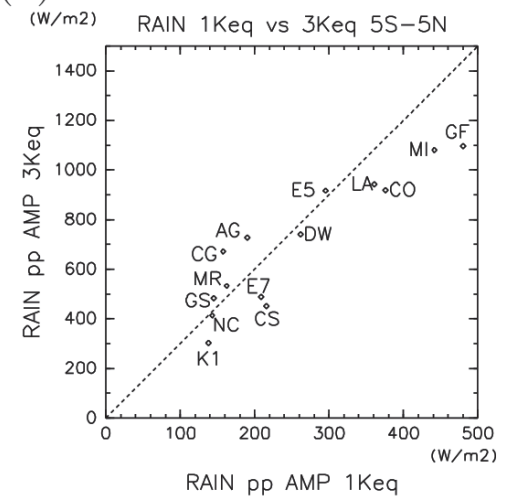

(b)

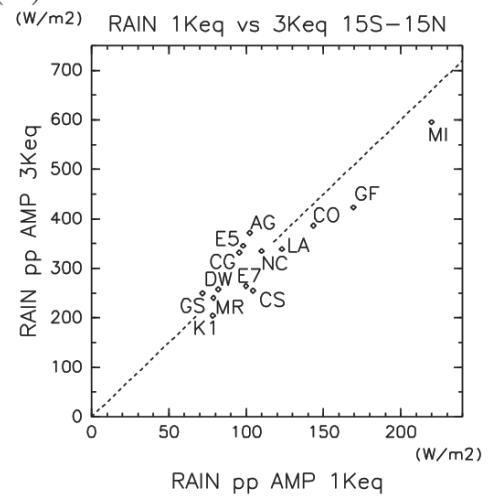

(c)

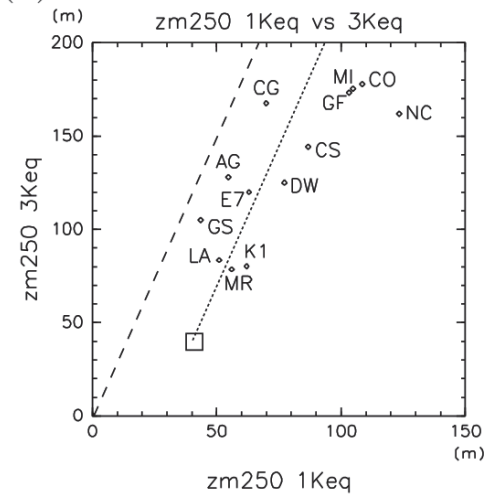

Fig. 16. Scatter plot comparing the peak-to-peak amplitudes of the anomalies in the 1KEQ and 3KEQ runs from the 15 APE models. (a) Precipitation anomaly averaged within $\pm 5^{\circ}$ of the equator. (b) Precipitation anomaly averaged within $\pm 15^{\circ}$ of the equator. (c) Mid-latitude geopotential height anomaly. The dotted lines in (a) and (b) show the amplitudes in 3KEQ as three times those in 1KEQ. In (c), the broken line corresponds to the same relationship as above, whereas the dotted line corresponds to a similar relationship, but the amplitudes in 3KEQ and $1 \mathrm{KEQ}$ have a common background value of $40 \mathrm{~m}$ noted by a square. See Table 1 for the label legends.

the anomalies from the CONTROL run. The Southern Hemisphere below a latitude of $-15^{\circ}$ is omitted because the response is mostly symmetric about the equator.

Forced by the zonal contrast of SST reaching 6 K (Fig. 1), precipitation in the tropics (Fig. 17a; see also Fig. 19n for the corresponding anomaly field) is mostly concentrated within the region of the warm SSTA. There is almost no rainfall over the cold SST region, where downward motion develops and the mid-troposphere becomes very dry (Fig. 17b, c). Mid-latitude precipitation, which is associated with the activity of baroclinic waves, shifts to lower latitudes and is enhanced around longitudes to the east of the SST maximum (Figs. 17a and 19n). Both the tropical and mid-latitude precipitation anomalies are represented as distinct zonal wavenumber one variations around the corresponding latitudes. It is also evident that the anomalies in the tropics and mid-latitudes are connected. A wavenumber one structure is also identified in the field of vertical motion on the equatorward flank of the baroclinic zone (Fig. 17c).

Corresponding to the intense longitudinal variation in the ITCZ precipitation, a wavenumber one, first baroclinic mode structure develops in the tropics, as seen in the mid-level temperature field (Fig. 17d) and the lower- and upper-level geopotential height fields (Fig. 17e,f; see also Figs. 21n and 22n for the corresponding anomaly fields). The maximum of the upper tropospheric high pressure in the tropics is located at longitudes between $30^{\circ}$ and $40^{\circ}$ to the east of the precipitation maximum (Fig. 17f). In the upper troposphere, wind is generally weak in the high pressure region around the equator, while a strong equatorial westerly wind develops in the cool SSTA region. The equatorial westerly wind bifurcates at the western tip of the high pressure around $(\lambda, \varphi) \sim\left(-60^{\circ}, 0^{\circ}\right)$ and merges at the eastern tip around $(\lambda, \varphi) \sim\left(110^{\circ}, 0^{\circ}\right)$. In contrast, in the lower troposphere, a pair of anticyclonic anomalies develop in the subtropics at longitudes to the west of the precipitation maximum centered at $(\lambda, \varphi) \sim$ $\left(-130^{\circ}, \pm 20^{\circ}\right)$ (Fig. 17e). The longitudinal variations in mid-tropospheric temperature; i.e., warm around $\lambda \sim$ $20^{\circ}$ and cool around $\lambda \sim-140^{\circ}$ (Fig. 17d), is consistent with that of the lower and upper geopotential variations mentioned above. Around the maximum of precipitation in the ITCZ, lower (upper) level zonal flow is divergent (convergent), whereas meridional flow is convergent (divergent) (Figs. 17e, f, 21n, and 22n).

The vertical structure of the zonal wave number one response in the tropics can be confirmed in Fig. $17 \mathrm{~g}$, which shows the equatorial section of zonal wind, vertical p-velocity, and temperature deviation from the zonal average. Both the temperature and vertical motion fields vary with zonal wavenumber one patterns, as was shown in the horizontal sections. There is strong upward motion over the longitudes of intense precipitation that extends over the full depth of the troposphere. On the other hand, the vertical motion outside the precipitation area is generally weak. The warm anomaly over the warm SST area observed in 


\section{KW1 Response (NCAR)}

(a) RAIN c.i. $=2 \mathrm{E}-5 \mathrm{~kg} \mathrm{~m}-2 \mathrm{~s}-1$

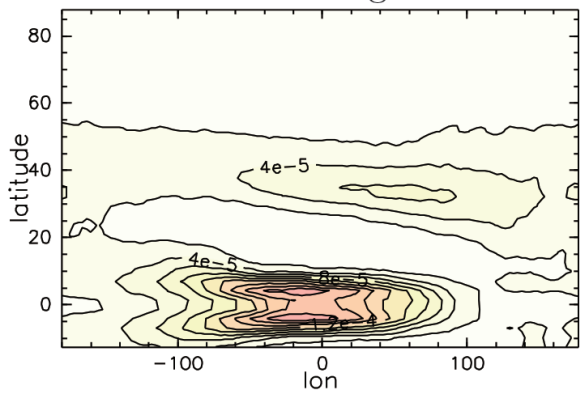

(c)

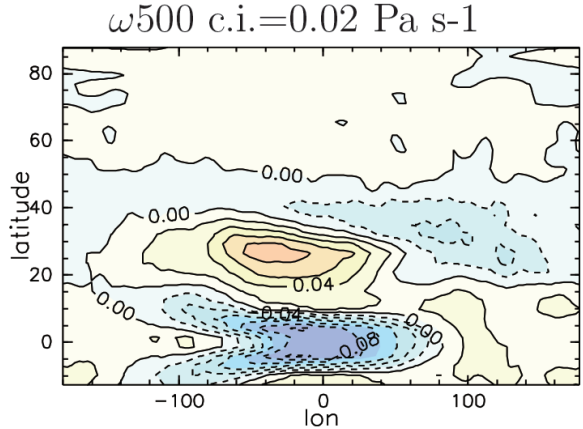

(e)

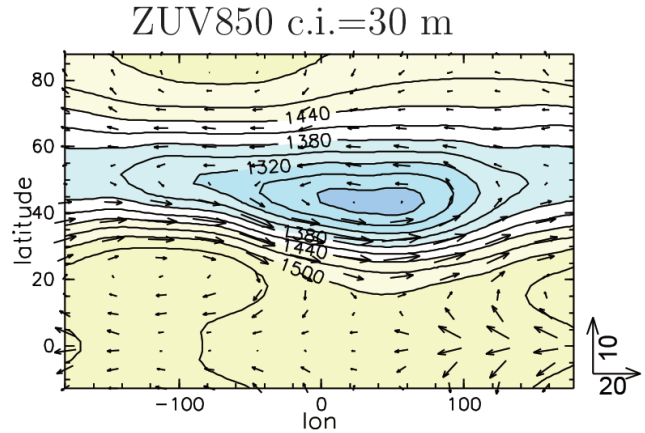

(g)

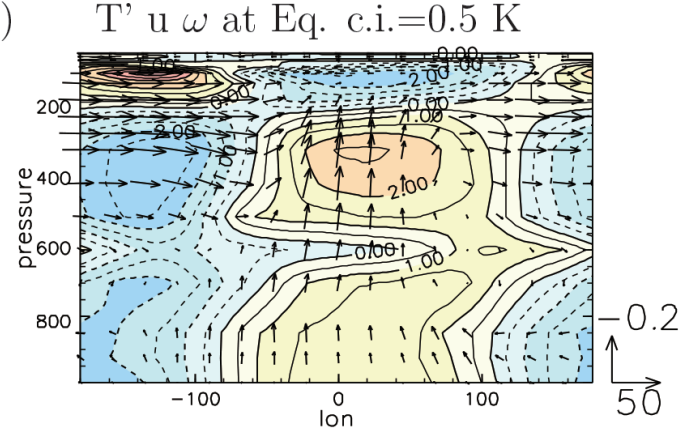

(b)

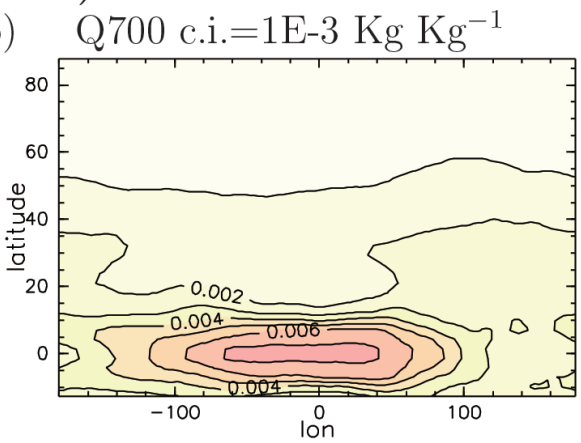

(d)

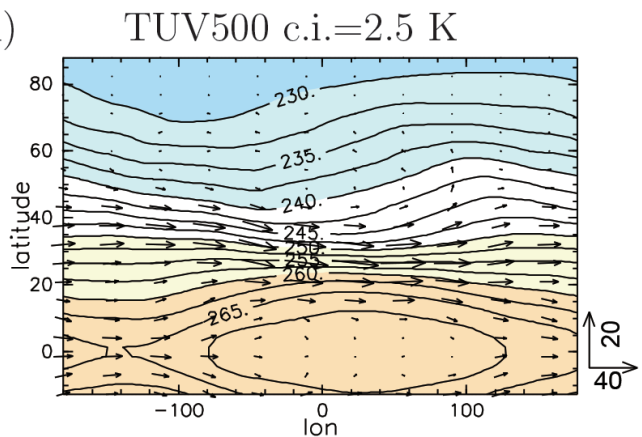

(f)

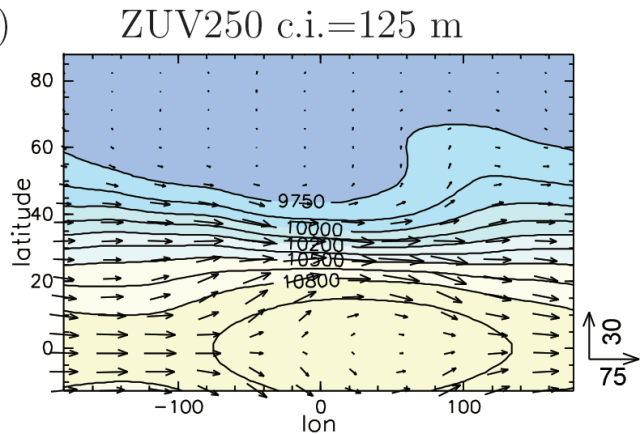

(h) abs.vort. 250 c.i. $=1 \mathrm{E}-5 \mathrm{~s}-1$

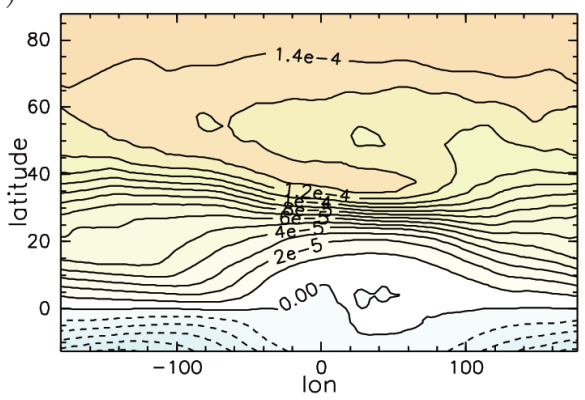

Fig. 17. Time mean response obtained from the $3 \mathrm{KW} 1$ run of NCAR. (a) Horizontal distribution of precipitation. (b) Water vapor mixing ratio at $700 \mathrm{hPa}$. (c) Pressure velocity at $500 \mathrm{hPa}$. (d) Temperature and horizontal wind at $500 \mathrm{hPa}$. (e) Geopotential height and horizontal wind at $850 \mathrm{hPa}$. (f) Geopotential height and horizontal wind at $250 \mathrm{hPa}$. (g) Temperature deviation from zonal mean, zonal wind, and vertical $p$-velocity at the equator. (h) Absolute vorticity at $250 \mathrm{hPa}$. Units and contour interval are indicated at the top of each panel. Magnitudes of the vector components are indicated to the right of each panel. Units: $\mathrm{m} \mathrm{s}^{-1}$ for horizontal components, and $\mathrm{Pa} \mathrm{s} \mathrm{s}^{-1}$ for $p$ velocity. 


\section{KW1 Anomaly of Ubar (NCAR)}

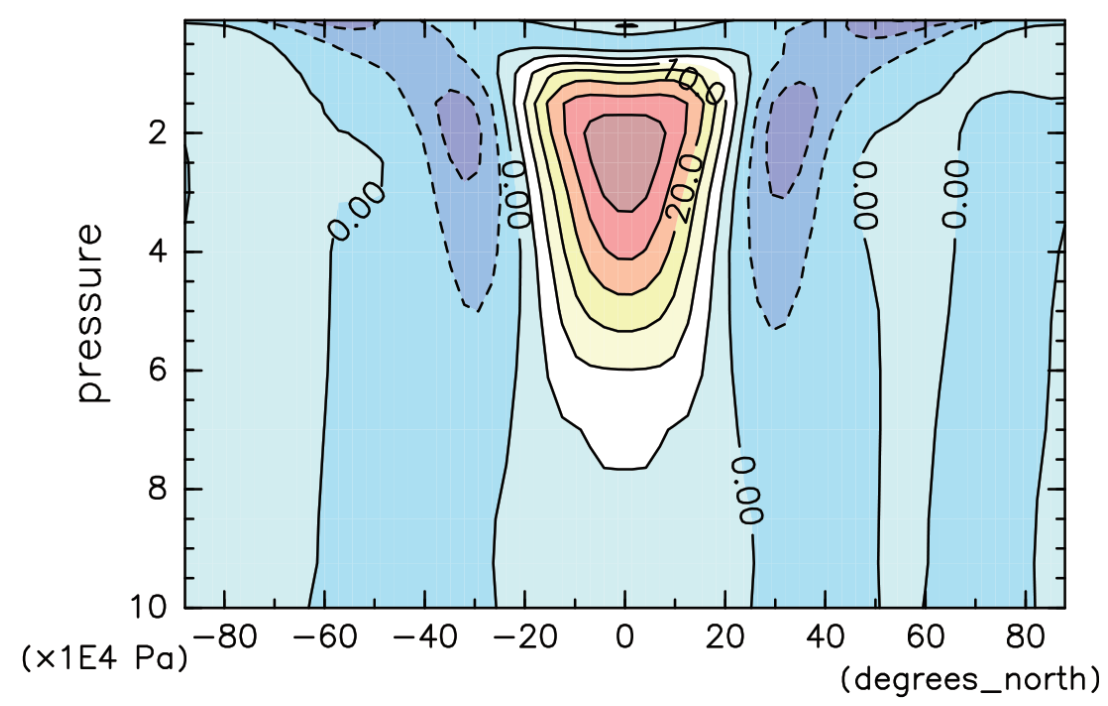

Fig. 18. Zonal mean zonal wind anomaly in the $3 \mathrm{KW} 1$ run of NCAR. Contour interval: $5 \mathrm{~m} \mathrm{~s}^{-1}$.

Fig. $17 \mathrm{~d}$ at the $500 \mathrm{hPa}$ level has a complex vertical structure; the signature of the anomaly is positive at the lower and the upper levels, but negative at the middle level, presumably due to the melting of the icy precipitation. There is a positive temperature anomaly around longitudes about $100^{\circ}$ to the east of the SSTA peak.

The westerly wind outside the warm region observed in Fig. $17 \mathrm{f}$ at the $250 \mathrm{hPa}$ level develops throughout the upper half of the troposphere. The same section for the same variables, but for the anomalies from the CONTROL experiment, is shown in Fig. 25n. There is one peculiar element worth noting in the vertical velocity anomaly: the raw vertical motion (Fig. 17g) is upward over the full depth of the troposphere, but its anomaly (Fig. 25n) appears only in the upper half of troposphere. The response of vertical velocity to the imposition of the SSTA is quite small at lower levels.

As was the case with the localized anomaly, 3KEQ, many of the characteristics of the tropical response summarized above contradict those expected from the classical linear theory of the thermal response to a wavenumber one equatorial heat source without background wind, where the off-equatorial upper-tropospheric high-pressure anomalies develop to the west of the heat source (e.g., fig. 9 in Matsuno 1966). In the NCAR 3KW1 run, the upper-tropospheric high-pressure region (Fig. 17f) seems to correspond to the zero potential vorticity area associated with the active convection; absolute vorticity is homogenized within the high pressure region in the upper troposphere as seen in Fig. 17h. However, an important difference from the zero potential vorticity region in the real atmosphere, which usually develops at, or to the west of, the convection center (Sardeshmukh and Hoskins 1985 ) is that it develops to the east of the convection center in the $3 \mathrm{KW} 1$ experiment. This is also thought to be related to the invasion of strong westerly winds in the upper troposphere observed in the APE CONTROL runs (see Section 3).

Comparing the horizontal distribution of the absolute vorticity in the upper troposphere in the $3 \mathrm{KW} 1$ (Fig. 17h) and CONTROL (Fig. 2c) runs, we notice that, near the equator, the meridional gradient of absolute vorticity in the upper troposphere in the cool SST region of $3 \mathrm{KW} 1$ is much steeper than that in CONTROL. As noted at the beginning of this subsection, there is almost no ITCZ precipitation in the region of the negative SSTA (Fig. 17a). Due to this drastic change in the pattern of precipitation, the upper tropospheric poleward flow of the Hadley cell also vanishes at the longitudes of the negative SSTA. Consequently, the equatorial air parcel with zero absolute vorticity cannot be transported over the wider latitudes around the equator. Instead, the air parcel from subtropical latitudes, with the larger absolute vorticity, is advected into the equatorial region. The extreme change in the tropical upper tropospheric absolute vorticity distribution in $3 \mathrm{KW} 1$ is in contrast to that in 3KEQ (Fig. 3h), 
where the extent of the change driven by the SSTA is restricted to modest wavy perturbations.

The extratropical response structure is also characterized by zonal wavenumber one. In the upper troposphere, the westerly jet shifts equatorward and strengthens in the longitudinal regions to the east of the SSTA maximum (Figs. $17 \mathrm{f}$ and $21 \mathrm{n}$, where the mid-tropospheric meridional temperature gradient is also enhanced (Fig. 17d). The activity of baroclinic waves is also enhanced along the intensified westerly jet, which is manifested as the low-level cyclonic region (Fig. 17e) and the upper-level trough (Fig. 17f) that develop to the north of the enhanced jet region to the east of the SSTA. At the longitudes of suppressed precipitation, the westerly jet is weak, but becomes broader to reach the equator (Fig. 17f), and considerably cooler air is advected from higher latitudes to longitudes around $\lambda=-180^{\circ} \sim-90^{\circ}$ (Fig. 17d). The invasion of westerly jets around the longitudes of the cold SSTA results in considerable acceleration of the zonal mean zonal wind. Figure 18 shows the meridional structure of the anomaly of zonal mean zonal wind in the $3 \mathrm{KW} 1$ run from NCAR compared with CONTROL. The westerly acceleration is centered in the equatorial upper troposphere, and confined within the Hadley cell. The low-latitude flank of the westerly jets are considerably decelerated in the upper troposphere, and stratospheric wind at higher latitudes is also decelerated.

In the anomaly from the CONTROL experiment (Fig. 21n), the strengthening and narrowing of the jet in the upper troposphere around the longitudes of the warm SSTA corresponds to the region of enhanced meridional geopotential gradient between the positive anomaly around the equator and the negative anomaly just to the north of the jet. Conversely, the weakening and broadening of the upper tropospheric jet around the the longitudes of cold SST is represented by the north-south oriented dipole geopotential anomaly centered around $\lambda=-150^{\circ}$, which is positive (negative) on the poleward (equatorward) side and straddles the mid-latitude westerly jet. In the lower troposphere, the development of the cyclonic anomaly just to the north of the baroclinic zone in the raw geopotential field at $850 \mathrm{hPa}$, and the meander of the westerly jet (Fig. $17 \mathrm{e})$ are represented as a distinct zonal wavenumber one anomaly of geopotential at, or slightly poleward, of the baroclinic zone (Fig. 22n). In the extratropics, poleward of the westerly jet, the vertical structure of the geopotential height anomaly is equivalent to barotropic, whereas it is baroclinic in the tropics.
5.2 Variety in the response among the 15 APE models a. Precipitation

In the following three subsections, we will compare the responses to the SSTA in $3 \mathrm{KW} 1$ experiment from the 15 APE models. As the responses are mostly symmetric about the equator for all of the APE models, the Southern Hemisphere below a latitude of $-15^{\circ}$ is omitted from the figures showing the horizontal structure of the responses. Latitudes higher than $60^{\circ}$ are also omitted because the precipitation anomalies there are weak.

In Fig. 19, we compare the time mean precipitation anomalies obtained from the 15 AGCM runs of APE. As is demonstrated by NCAR in the previous subsection, zonal wavenumber one patterns are evident across a wide range of latitudes in all of the models. The dominance of wavenumber one is shared among variables other than precipitation for all models. There are three latitudinal bands where the response is notable: the ITCZ around the equator, and the two mid-latitude baroclinic zones (one in each hemisphere). Additional features that are also common to all models are indicated below. Unlike the 3KEQ SSTA, the wavenumber 5 variation along the baroclinic zones can not be identified, or is overshadowed by the wavenumber one anomaly, which is much stronger than the eastwest dipole in $3 \mathrm{KEQ}$. At higher latitudes, precipitation decreases around $\lambda=0^{\circ}$ and increases around $\lambda=180^{\circ}$ along $\varphi \sim \pm 50^{\circ}$ in the majority of the models.

The meridional structure of the precipitation anomaly in the ITCZ varies among the models. The models can be classified into three categories with respect to the positive anomaly pattern over the warm SSTA. First, CSIROstd has two distinct zonally elongated maxima along latitudes around $\pm 6^{\circ}$. Second, ECMWF05 and ECMWF07 exhibit an intense maximum along the equator associated with a pair of negative anomaly bands; this unusual feature is presumably due to the narrow meridional scale of the SSTA unintentionally used in these two ECMWF experiments (see Section 2 ). The equatorial concentration of precipitation is quite intense in ECMWF05, but less so in ECMWF07, as will be shown below. Third, in the other models, the positive anomaly is most intense along the equator, but has some meridional extent. Focusing on the negative anomaly pattern over the cool SSTA, the models can be classified into two categories. In CGAM, GSFC, K1JAPAN, NCAR, and UKMOn96, the reduction in precipitation is greatest in the two latitude bands off the equator. In the other models, it is most pronounced at the equator. The meridional structure of the negative anomaly of a particular $3 \mathrm{KW} 1$ run reflects the 
3KW1 Precipitation Anomalies

(a) AGU

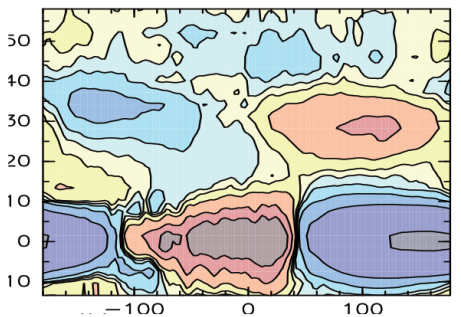

(d) CSIROold

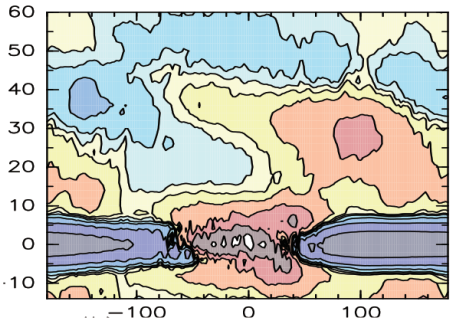

(g) ECMWF07

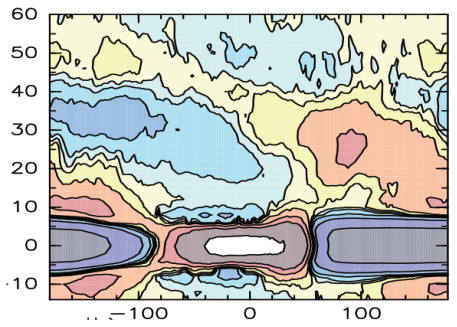

(j) K1JAPAN

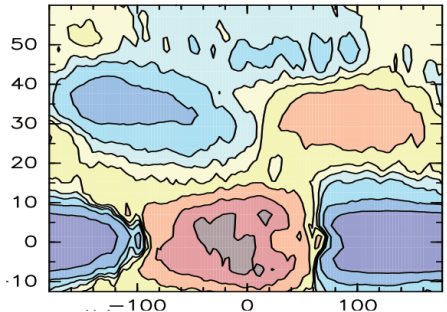

(m) MRI

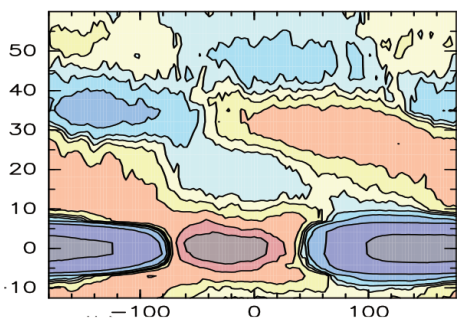

(b) CGAM

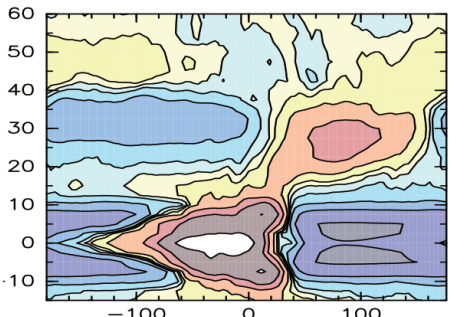

(e) DWD

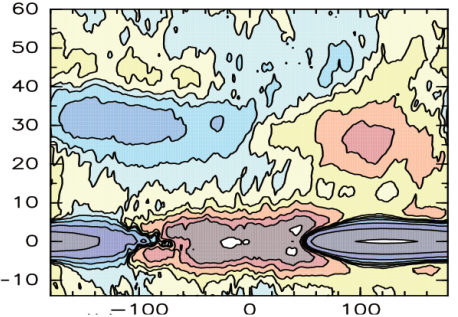

(h) GFDL

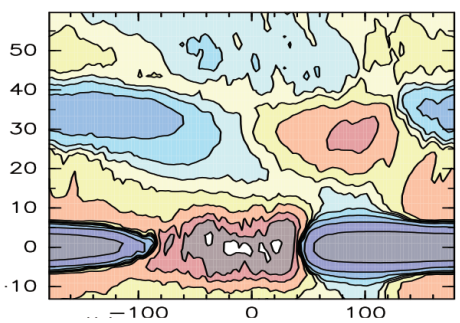

(k) LASG

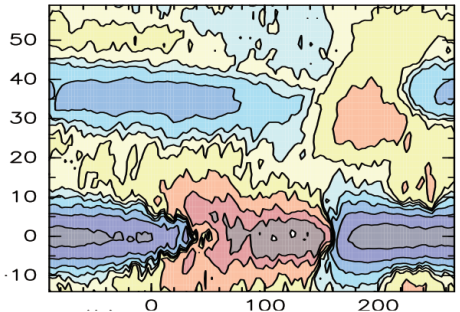

(n) NCAR

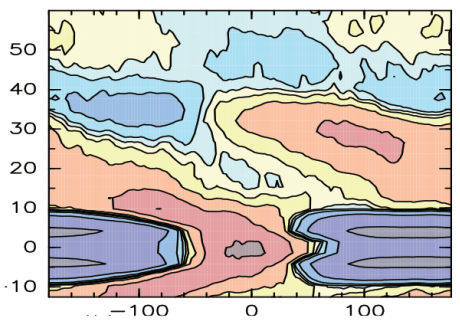

(c) CSIROstd

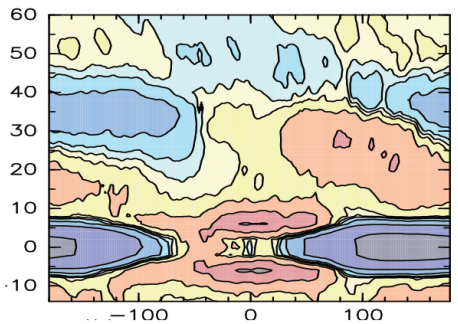

(f) ECMWF05

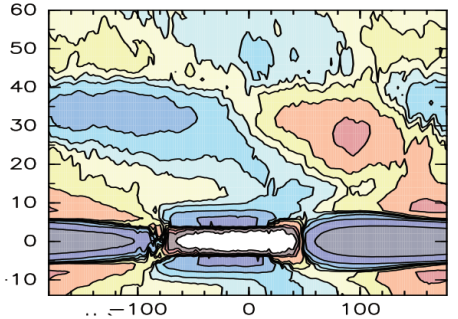

(i) GSFC

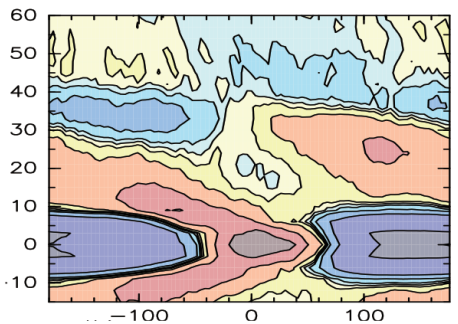

(l) MIT

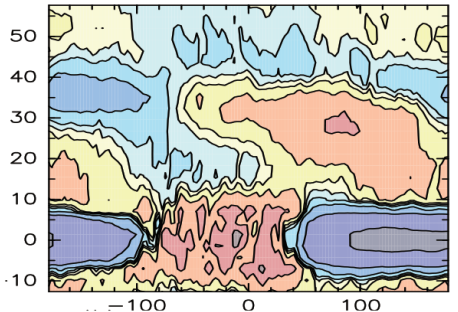

(o) UKMOn96

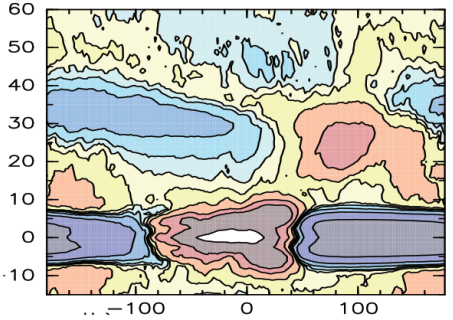

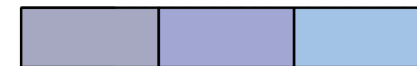

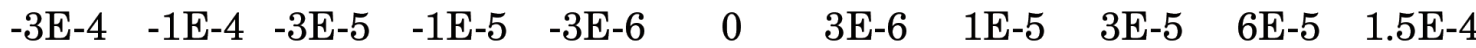

Fig. 19. As Fig. 4 but for the 3KW1 runs. Note that shading for greater than $6 \times 10^{-5} \mathrm{~kg} \mathrm{~m}^{-2} \mathrm{~s}^{-1}$ differs from Fig. 4 . 
meridional structure of the ITCZ in the corresponding CONTROL run. The precipitation anomaly in $3 \mathrm{KW} 1$ is so strong that precipitation over the cool SST area is almost completely suppressed (e.g., Fig. 17a for NCAR). As a result, the precipitation anomaly there becomes simply the rainfall in CONTROL, but with a negative signature.

Focusing on the behavior of precipitation in the subtropics, the models can be classified into two groups. In GSFC, MRI, NCAR, and also in ECMWF07, there are noticeable anomalies in the subtropics that bridge the equatorial and mid-latitude anomalies. The anomaly pattern, tilted from south-east to north-west in the Northern Hemisphere, suggests the presence of Rossby waves propagating from the tropics to the higher latitudes. In other models, such features are weak or absent.

The intensity of the precipitation anomalies around the ITCZ and the baroclinic zone in each of the $15 \mathrm{APE}$ models is summarized in Fig. 20 and Table 3. In the left column of Fig. 20, the zonal distributions of precipitation anomalies along the equator are listed. Excluding the two ECMWF experiments that are undoubtedly affected by the off-specified narrow meridional scale of the SSTA, the peak-to-peak amplitude varies by a factor of as much as five from the weakest in CSIROstd to the strongest in DWD. In addition, the precipitation maximum is not necessarily located at the position of the highest SST. In the majority of the models, the precipitation peaks are located to the west of the SST peak at longitudes between $-10^{\circ}$ and $-40^{\circ}$. An exception is LASG, in which the precipitation peak is shifted to the east.

If we compare the precipitation anomalies normalized by the precipitation obtained from the CONTROL run for each model, scattering among the models is reduced considerably. As shown in the first and second columns of Table 3 for $3 \mathrm{KW} 1$, the maximum values range from $115 \%$ to $282 \%$, and the minimum values from $9 \%$ to $42 \%$ of the CONTROL runs on the equator, excluding the two ECMWF experiments for which the meridional width of the SSTA is half that of the other models. As for those models with the double peaked ITCZ structure, the scaled responses in the off-equatorial latitudes are shown in the third and fourth columns of Table 3). In CSIROstd and K1JAPAN, the scaled positive precipitation anomalies are stronger than those at the equator. In CSIROstd, the amount of precipitation at the off-equatorial maxima is about four times that of CONTROL, resulting in the distinct meridional splitting of the positive precipitation anomalies (Fig. 19c). In CGAM and K1JAPAN, the negative anoma- lies are more pronounced than those at the equator.

The central column of Fig. 20 shows the longitudinal distributions of the precipitation anomalies from the 15 models meridionally averaged over an equatorial band within $\pm 15^{\circ}$. While the variability of the meridional structures is eliminated by the meridional averaging, a considerable longitudinal variation among the models remains. The scaled response in the same latitudinal band listed in the sixth and seventh columns of Table 3 also confirms the reduction in scattering among the models; the maxima are about $200 \%$, and the minima about $30 \%$ of the CONTROL runs. The shape of the zonal variations of precipitation is sawtooth-like in the majority of the models; moving east, it increases slowly before decreasing steeply.

In the right column of Fig. 20, the meridional distribution of the precipitation anomalies in the mid-latitude baroclinic zone averaged between $20^{\circ} \mathrm{N}$ and $40^{\circ} \mathrm{N}$ are plotted. They are dominated by the wavenumber one variation in all of the models. The peaks are located at longitudes around $\lambda=90^{\circ}$ in most of the models, and their amplitudes vary little among the models. This similarity among the mid-latitude precipitation responses contrasts with the much larger differences found in the $3 \mathrm{KEQ}$ runs listed in the right column of Fig. 5. In the scaled variation listed in the last two columns of Table 3 , the amplitudes of the mid-latitude average precipitation anomalies are around $60 \%$ of the CONTROL runs in most of the models, which are about three times those in 3KEQ.

\section{b. Horizontal structure of dynamic fields}

The horizontal structures of the responses, namely the anomaly fields of horizontal wind and geopotential height, on the $250 \mathrm{hPa}$ and the $850 \mathrm{hPa}$ surfaces are shown in Figs. 21 and 22 for all models. As the responses are mostly symmetric about the equator, the Southern Hemisphere below a latitude of $-15^{\circ}$ is omitted.

The major features of the response described for NCAR in Section 5.1 are common among the models, although the intensities, locations, and other details of the anomaly patterns do differ. At the $250 \mathrm{hPa}$ level (Fig. 21), a positive geopotential anomaly appears on the eastern side of the warm SSTA longitudes, a northsouth oriented dipole anomaly centered around the longitude of $\lambda \sim-150^{\circ}$ develops straddling the midlatitude westerly jet, and a negative anomaly appears just poleward of the jet around the longitudes of the SSTA. At the $850 \mathrm{hPa}$ level (Fig. 22), a distinct zonal wavenumber one anomaly of geopotential at, or slightly poleward, of the baroclinic zone can be seen. A posi- 


\section{KW1 Precipitation Response in Selected Latitude Bands}
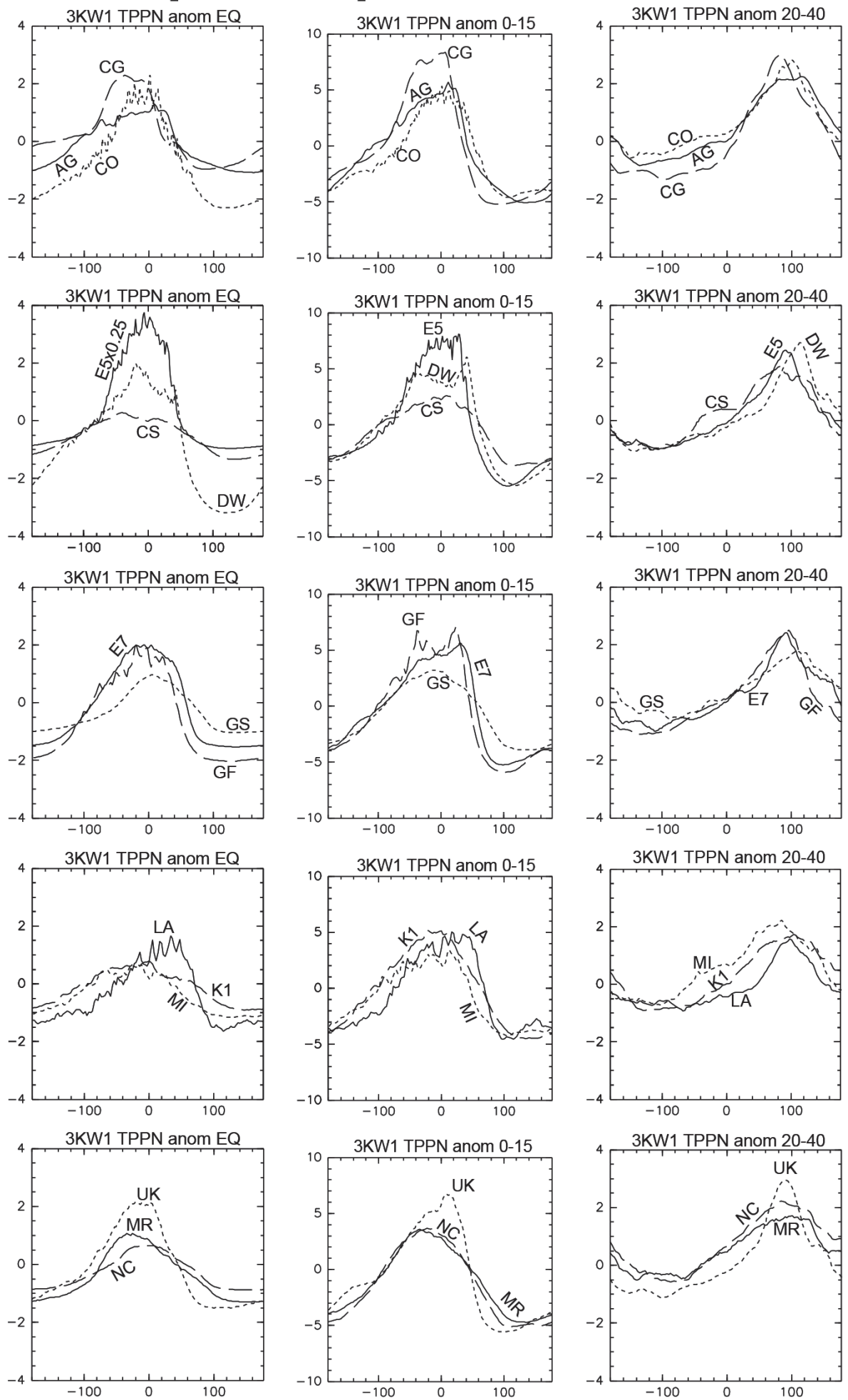

Fig. 20. As Fig. 5 but for the $3 \mathrm{KW} 1$ runs. Units are $10^{-4} \mathrm{~kg} \mathrm{~m}^{-2} \mathrm{~s}^{-1}$ for left panels, and $10^{-5} \mathrm{~kg} \mathrm{~m}^{-2} \mathrm{~s}^{-1}$ for center and right panels. See Table 1 for the label legends. 
Table 3. 3KW1 Response of Precipitation normalized by CONTROL

\begin{tabular}{lccccccccc}
\hline \multicolumn{1}{c}{$\begin{array}{c}\text { model } \\
\text { symbol }\end{array}$} & \multicolumn{2}{c}{ equator $\%$} & \multicolumn{3}{c}{ off-equator $\%$} & \multicolumn{3}{c}{ ave. in $15 \mathrm{~S}-15 \mathrm{~N} \%$} & \multicolumn{2}{c}{ ave. in 20N-40N \% } \\
& $\min$ & $\max$ & $\min$ & $\max$ & lat. & $\min$ & $\max$ & $\min$ & $\max$ \\
\hline AGU & 23 & 193 & - & - & - & 26 & 181 & 68 & 184 \\
CGAM & 24 & 282 & 13 & 199 & 4 & 19 & 227 & 55 & 199 \\
CSIROstd & 29 & 115 & 95 & 422 & 8 & 47 & 144 & 63 & 159 \\
CSIROold & 17 & 183 & - & - & - & 35 & 171 & 73 & 235 \\
DWD & 10 & 156 & - & - & - & 24 & 186 & 66 & 189 \\
\hline ECMWF05 & 9 & 456 & - & - & - & 29 & 207 & 67 & 191 \\
ECMWF07 & 9 & 219 & - & - & - & 24 & 177 & 67 & 173 \\
GFDL & 13 & 184 & - & - & - & 25 & 186 & 63 & 186 \\
GSFC & 9 & 185 & 9 & 162 & 6 & 26 & 200 & 82 & 159 \\
K1JAPAN & 42 & 150 & 17 & 247 & 5 & 34 & 180 & 62 & 170 \\
\hline LASG & 37 & 164 & - & - & - & 40 & 165 & 55 & 179 \\
MIT & 27 & 141 & - & - & - & 42 & 142 & 75 & 195 \\
MRI & 17 & 169 & - & - & - & 24 & 156 & 82 & 171 \\
NCAR & 13 & 164 & 13 & 138 & 6 & 25 & 157 & 72 & 184 \\
UKMOn96 & 15 & 223 & 14 & 182 & 3 & 22 & 200 & 61 & 203 \\
\hline
\end{tabular}

*Meridional scale of SSTA is halved.

tive anomaly of zonal mean zonal wind in the tropical upper troposphere, like that observed in NCAR (Fig. 18), also develops in most of the models. However, as will be discussed later, their intensities vary significantly among the models. The invasion of a westerly wind into the equatorial region around the longitudes of the cold SSTA is, as was mentioned for NCAR, thought to be responsible for the acceleration of the zonal wind (Fig. 21). A further noteworthy feature is that a trace of Rossby wave propagation from the tropics to the mid-latitude baroclinic zone can be found in CSIROold, GSFC, MRI, and NCAR at the $250 \mathrm{hPa}$ level (Figs. 21d,i,m and 21n, respectively); a series of geopotential height anomalies continue in the subtropics, with a westward phase tilt from lower to higher latitudes.

As the responses in the $3 \mathrm{KW} 1$ experiments described above are dominated by zonal wavenumber one patterns, we now focus on the amplitudes and phases of the wavenumber one components of selected variables. Figure 23a compares the amplitudes and phases of the precipitation anomalies averaged in the latitudinal band between $\pm 15^{\circ}$. Although the amplitudes vary by a factor of about two, the phases are well concentrated within a longitudinal range of $30^{\circ}$. In most of the models, the precipitation response shifts longitudinally to the west by about $30^{\circ}$ from the SST variation. Figures $23 \mathrm{~b}$, c, and d compare the amplitudes and phases of the geopotential anomalies at the $250 \mathrm{hPa}$ level at latitudes of $20^{\circ}, 40^{\circ}$, and $60^{\circ}$, respectively. The amplitudes and phases at $20^{\circ}$ are distributed in a fairly compact region, which is located slightly to the east of the SSTA; the relative scattering of the amplitudes appears to be less than that for precipitation (Fig. 23a). At $40^{\circ}$, the phases of the geopotential anomalies are scattered considerably, but scattering of the amplitudes is still within $30 \%$ of its average value. However, at $60^{\circ}$, the phases of the geopotential anomalies are scattered over a wide range, the phase variation reaches almost $90^{\circ}$, and the amplitudes vary by more than a factor of five. One might imagine that the increased scattering of wave properties at higher latitudes is natural because of the naive nature of Rossby wave propagation and the increased geopotential magnitude of variations in geostrophic phenomena as latitude increases. However, quantitative analysis of these issues remains to be performed.

\section{c. Multi-model statistics of the response}

Figure 24a shows the model mean response of geopotential and horizontal wind vectors at $250 \mathrm{hPa}$, and Fig. $24 \mathrm{~b}$ shows the standard deviation of the geopotential anomalies for $3 \mathrm{KW} 1$ at $250 \mathrm{hPa}$ from the $15 \mathrm{APE}$ models. Figures $24 \mathrm{c}$, d show those at $850 \mathrm{hPa}$. As the responses for $3 \mathrm{KW} 1$ in the 15 APE models commonly shared a zonally wavenumber one structure, which is directly represented in the model mean response, it is natural that the multi-model mean response is also characterized with zonally wavenumber one structure. However, it is notable that the distribution of the stan- 
3KW1 Anomalies of Geopotential height, $\mathrm{u}$, and $\mathrm{v}$ at $250 \mathrm{hPa}$

(a) $\mathrm{AGU}$

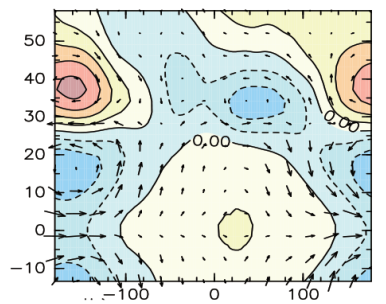

(d) CSIROold

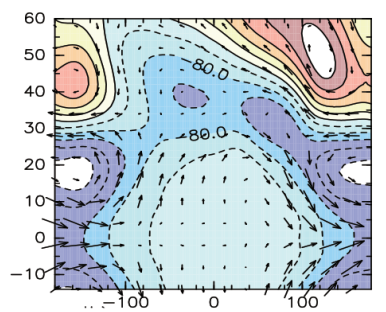

(g) ECMWF07

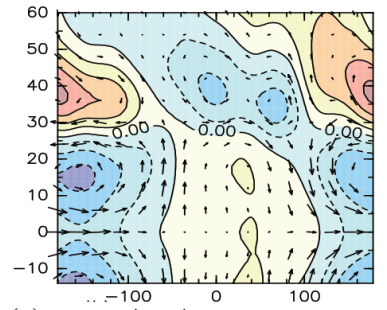

(j) K1JAPAN

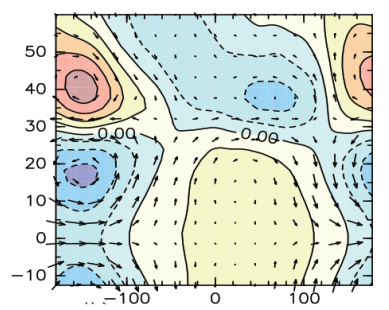

(m) MRI
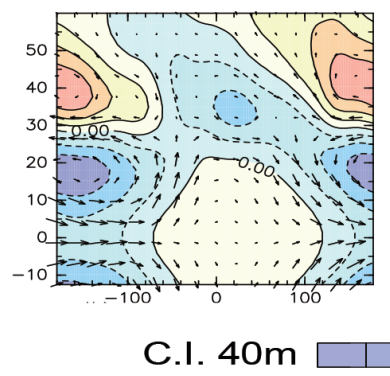

(b) CGAM

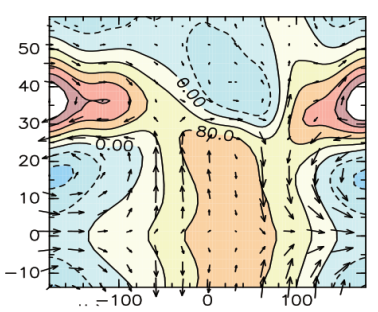

(e) DWD

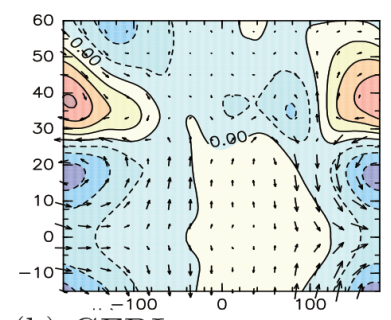

(h) GFDL

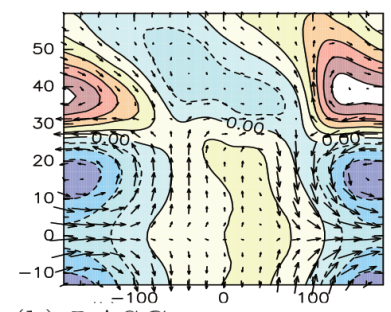

(k) LASG

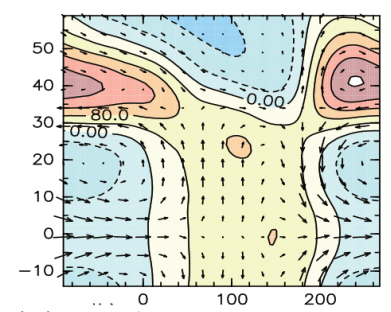

(n) NCAR

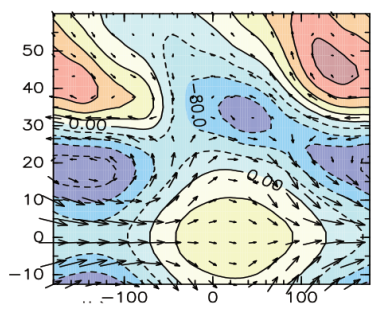

(c) CSIROstd

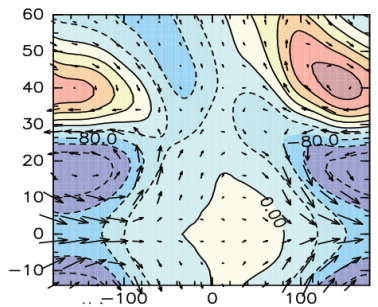

(f) ECMWF05

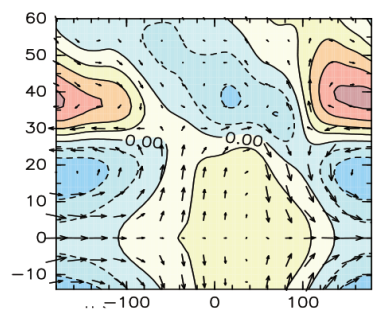

(i) GSFC

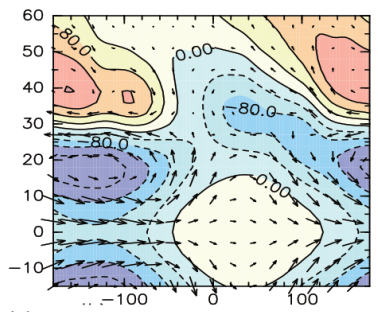

(1) $\mathrm{MIT}$

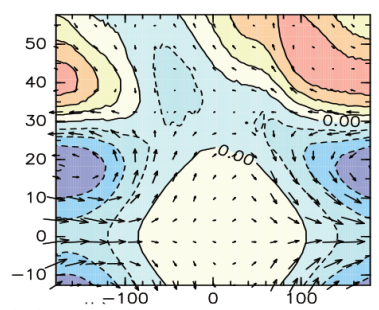

(o) UKMOn96

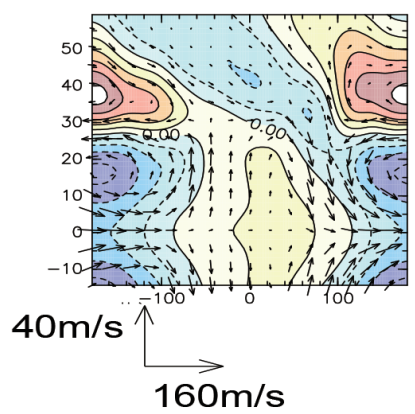

Fig. 21. Time mean anomalies of geopotential height and horizontal velocity vector at $250 \mathrm{hPa}$ in the $3 \mathrm{KW} 1$ runs from the 15 APE models. Contour interval and magnitudes of wind vector components are indicated at the bottom. 
3KW1 Anomalies of Geopotential height, u, and v at $850 \mathrm{hPa}$

(a) AGU

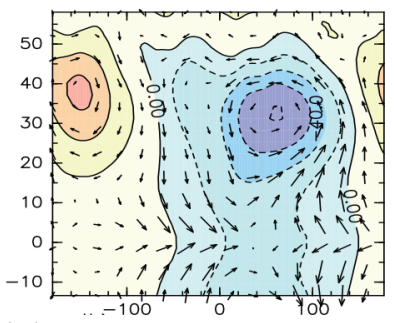

(d) CSIROold

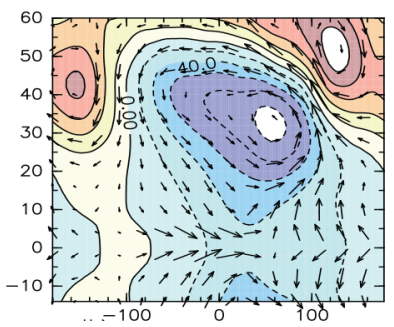

(g) ECMWF07

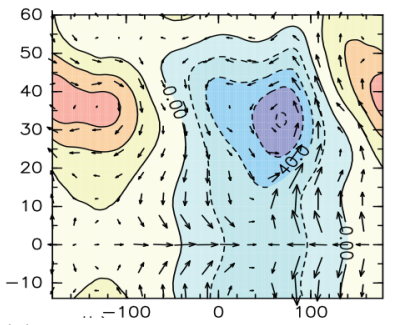

(j) K1JAPAN

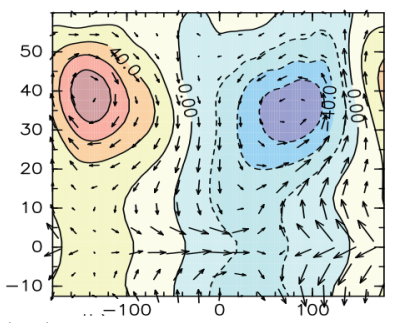

(m) MRI

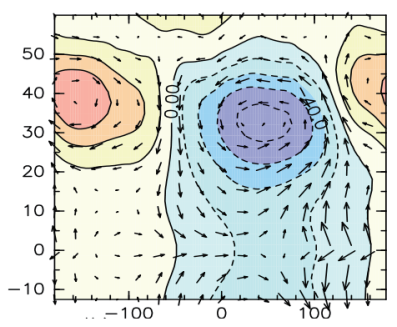

(b) CGAM

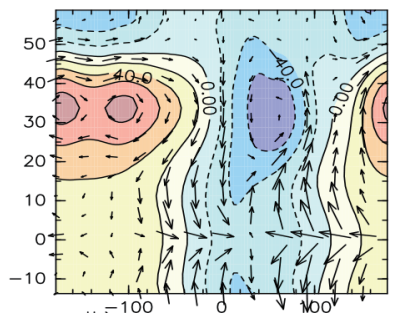

(e) DWD

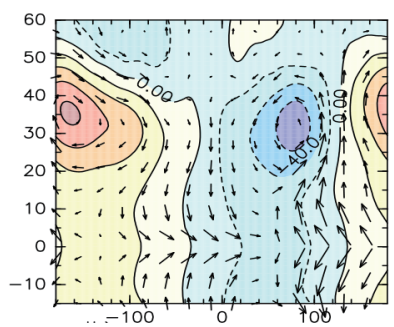

(h) GFDL

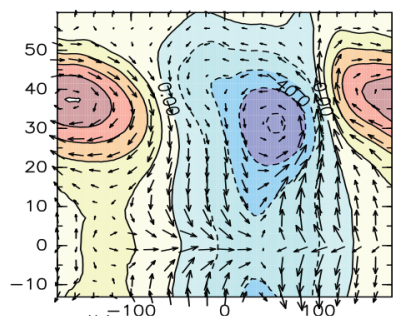

(k) LASG

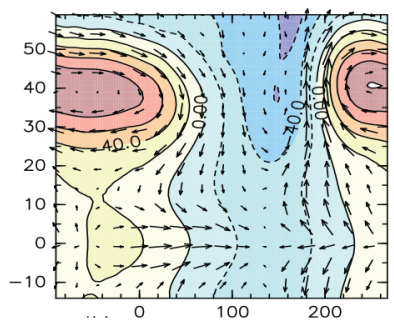

(n) NCAR

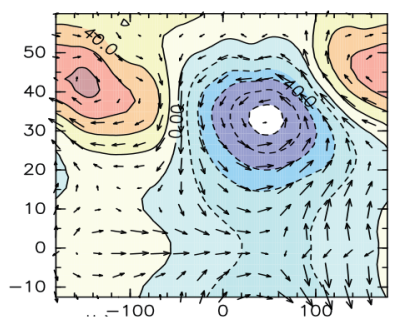

C.I. $20 \mathrm{~m}$

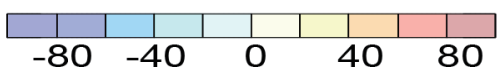

(c) CSIROstd

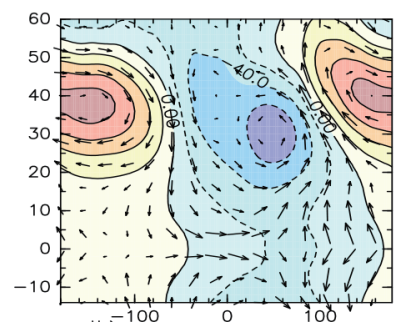

(f) ECMWF05

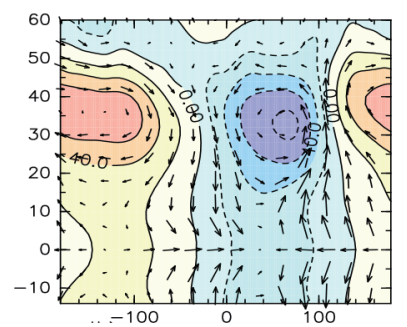

(i) GSFC

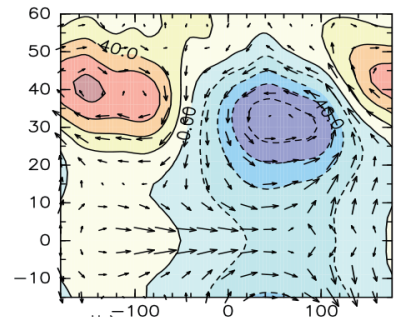

(l) $\mathrm{MIT}$

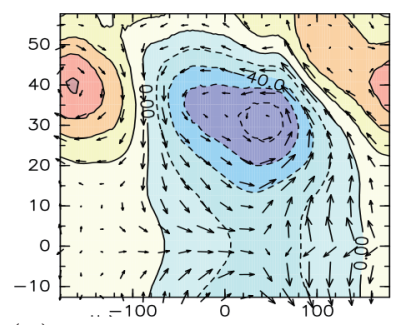

(o) UKMOn96

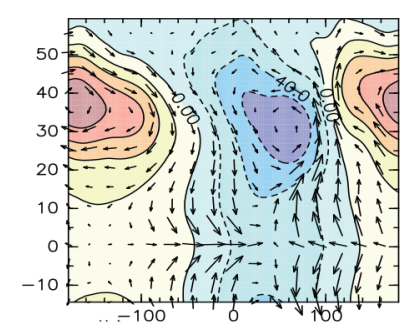

$10 \mathrm{~m} / \mathrm{s} /$

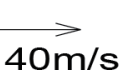

Fig. 22. As Fig. 21 but for $850 \mathrm{hPa}$. 
(a)

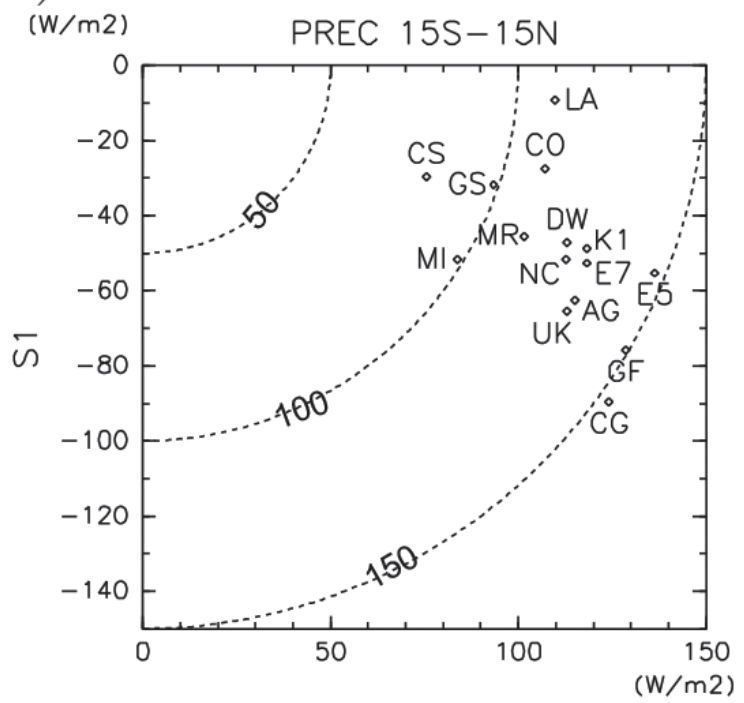

C1

(c)

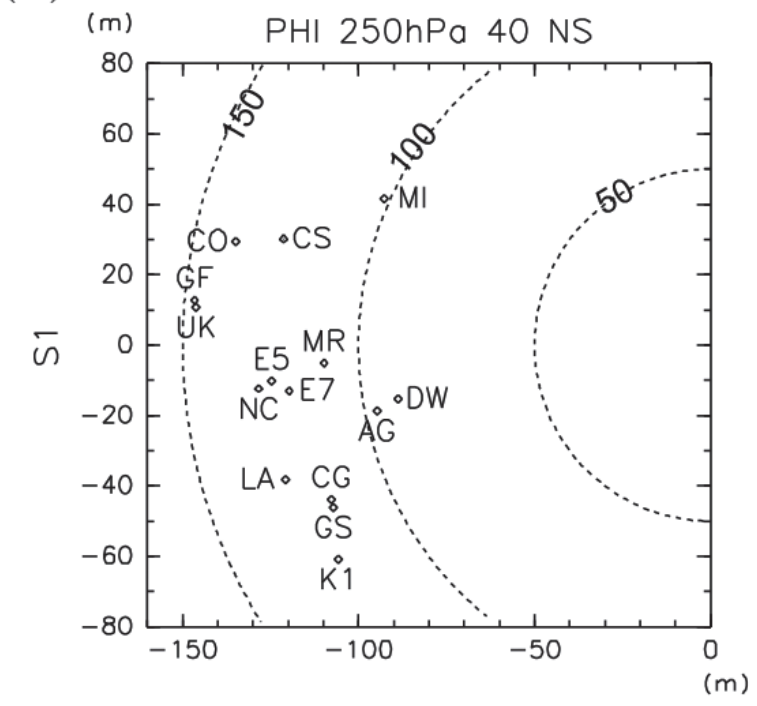

C1 (b)

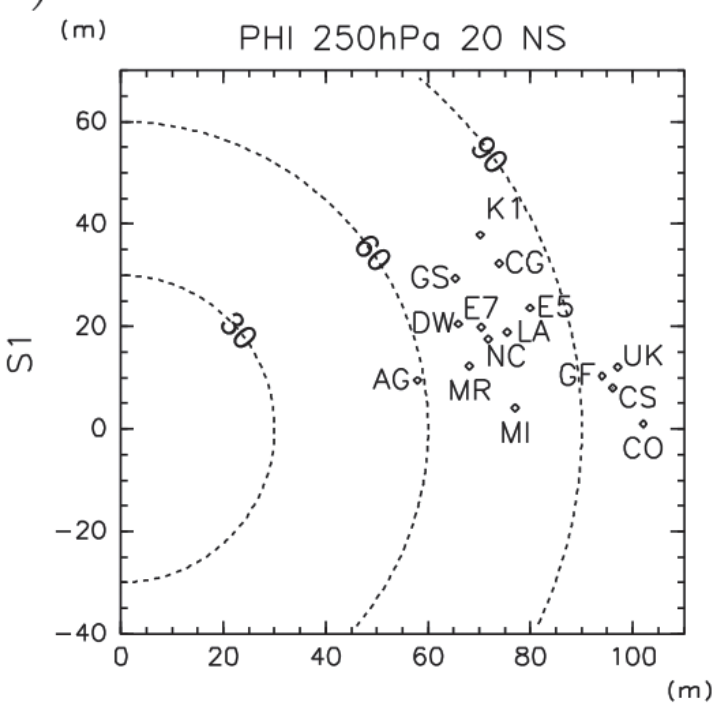

C1

(d)

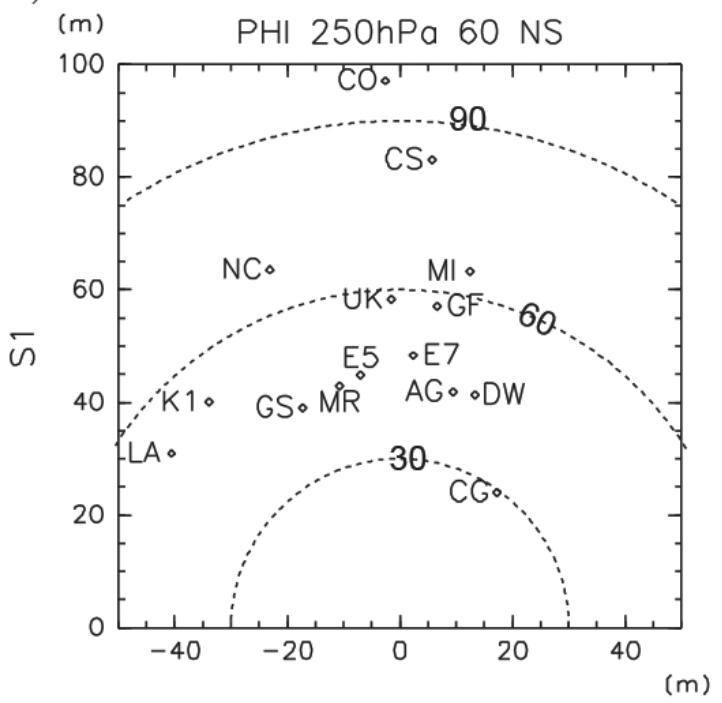

C1

Fig. 23. Scatter plots showing the sine (s1) and cosine (c1) coefficients of wavenumber one components of variables in the $3 \mathrm{KW} 1$ runs from the $15 \mathrm{APE}$ models. (a) Precipitation averaged within $\pm 15^{\circ}$ of the equator. (b) Geopotential height at $20^{\circ} \mathrm{N}$. (c) Geopotential height at $40^{\circ} \mathrm{N}$. (d) Geopotential height at $60^{\circ} \mathrm{N}$. Dashed lines indicate magnitudes. See Table 1 for the label legends.

dard deviations has considerable longitudinal inhomogeneity. At low latitudes, the scattering of the upper tropospheric geopotential anomaly among the models (Fig. 24b) is smaller (larger) in the region of the high (low) pressure anomaly. Such correlation is absent at lower levels (Fig. 24d). In the extratropics, the locations of enhanced scattering in the lower and upper levels nearly coincide, presumably due to the generally barotropic structure of the response in the individual models. 


\section{KW1 Multi-Model Statistics of the Response}

(a) model mean ZUV250

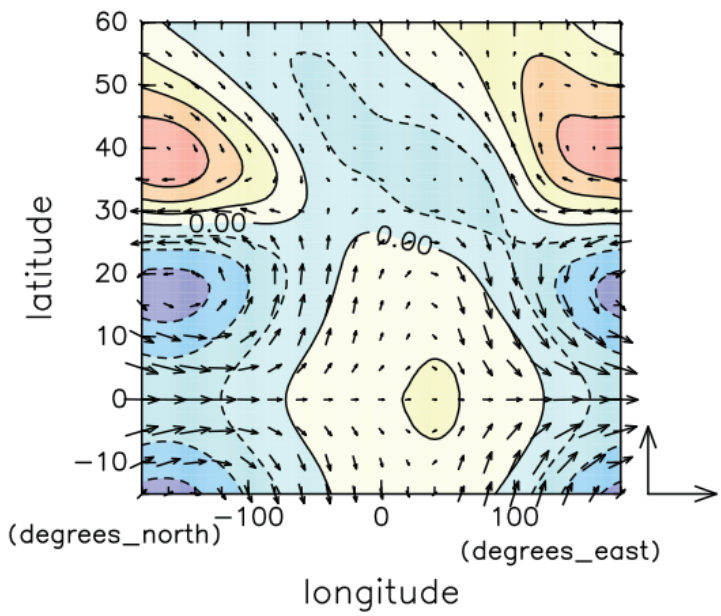

(c) model mean ZUV850

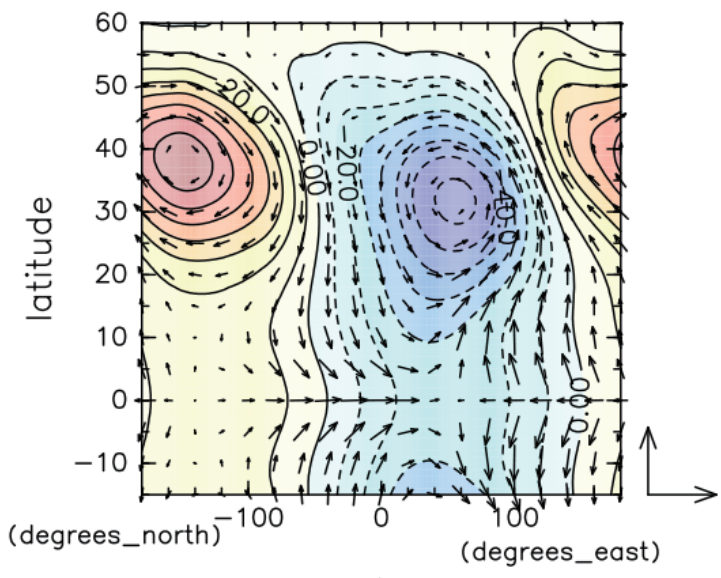

longitude (b) standard deviation Z250

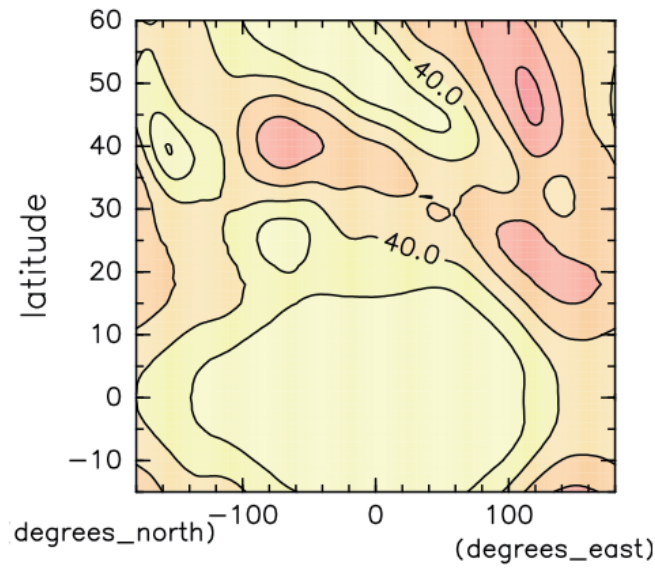

longitude

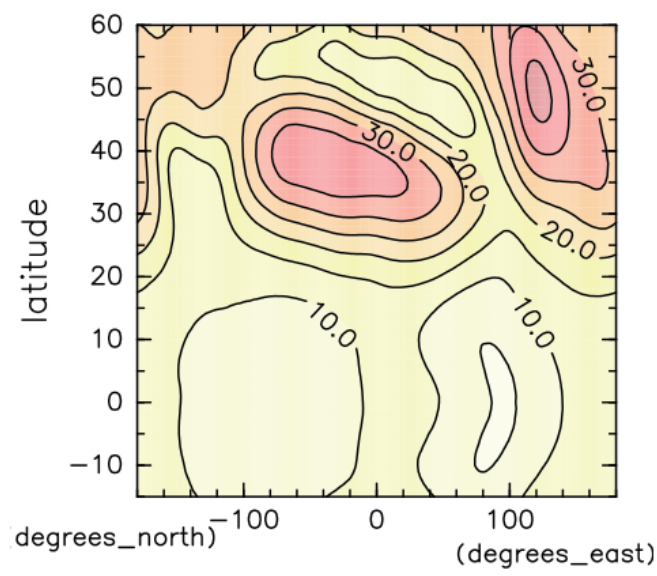

longitude

Fig. 24. Time mean anomalies of multi-model statistics of the response in the $3 \mathrm{KW} 1$ runs from the 15 APE models. (a) Model averages of the temporal mean anomalies of geopotential height and horizontal velocity vector at 250 $\mathrm{hPa}$. Contour interval: $40 \mathrm{~m}$. The unit vectors of zonal and meridional wind are $80 \mathrm{~m} \mathrm{~s}^{-1}$ and $20 \mathrm{~m} \mathrm{~s}^{-1}$, respectively. (b) The standard deviation of the temporal mean anomalies of geopotential height at $250 \mathrm{hPa}$. Contour interval: $10 \mathrm{~m}$. (c) As (a) but for $850 \mathrm{hPa}$. Contour interval: $10 \mathrm{~m}$. The unit vectors of zonal and meridional wind are $20 \mathrm{~m} \mathrm{~s}^{-1}$ and $5 \mathrm{~m} \mathrm{~s}^{-1}$, respectively. (d) As (b) but for $850 \mathrm{hPa}$. Contour interval: $5 \mathrm{~m}$.

\section{d. Vertical structure along the equator}

Figure 25 shows the vertical sections of the anomalies of temperature, zonal wind, and vertical p-velocity at the equator from the $15 \mathrm{APE}$ models. Figure 26 shows the vertical sections of the anomalies of temperature tendency due to the sum of parameterized and resolved cloud processes from the nine APE models for which data are available. Figures 27 a to 27 i show the vertical distributions of the anomalies of temperature tendency due to parameterized and resolved cloud processes at the maxima of the precipitation anomalies in the same nine APE models. The anomalies of temperature, 
3KW1 Anomalies of $\mathrm{T}, \mathbf{u}$, and $\omega$ at the Equator

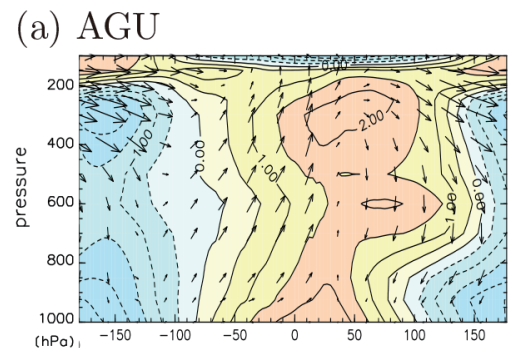

(d) CSIROold

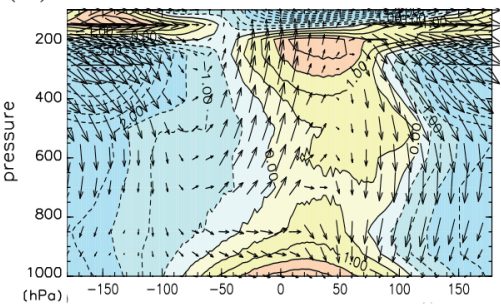

(g) ECMWF07

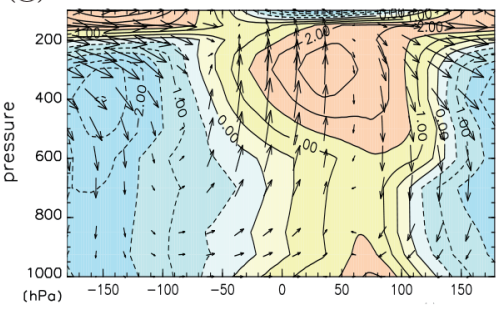

(j) K1JAPAN

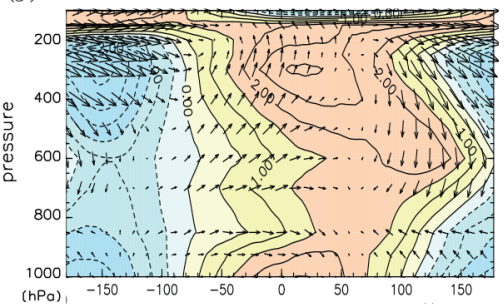

(m) MRI

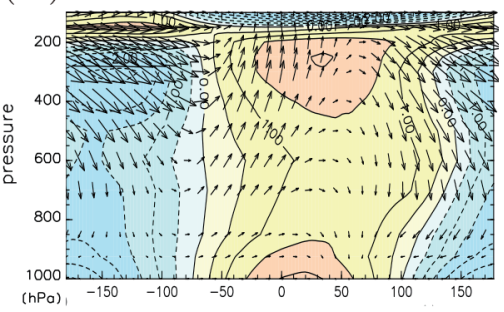

(b) CGAM

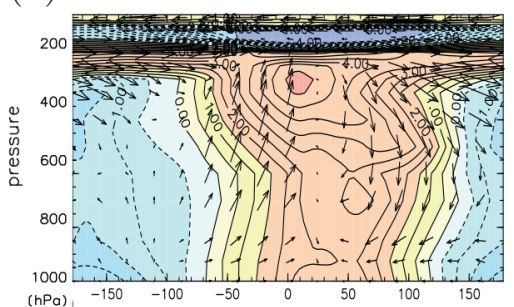

(e) DWD

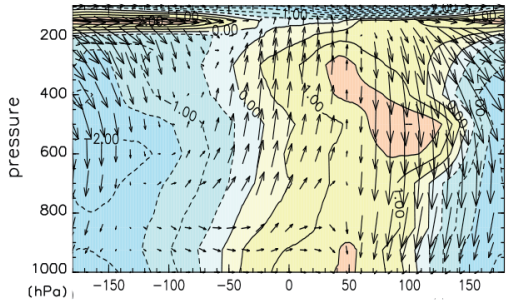

(h) GFDL

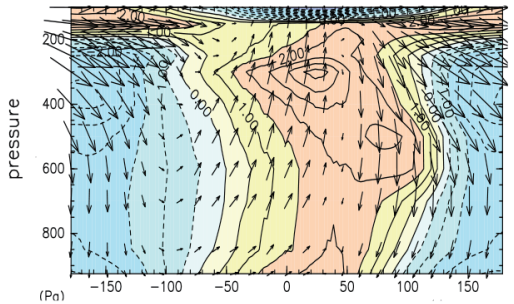

(k) LASG

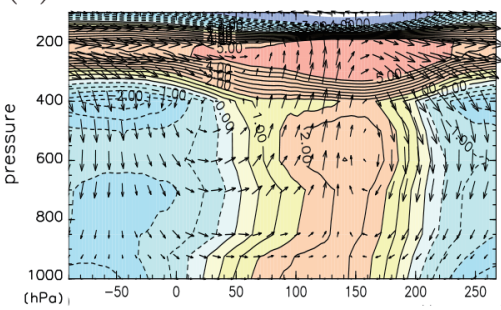

(n) NCAR

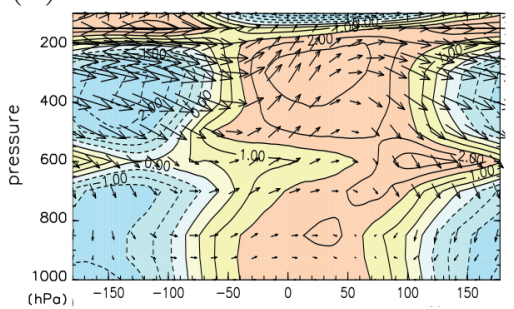

(c) CSIROstd

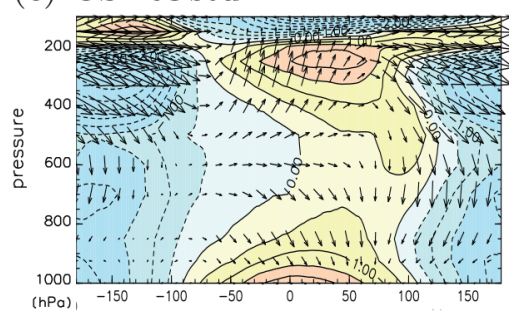

(f) ECMWF05

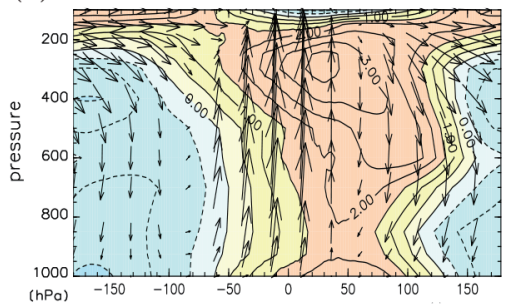

(i) GSFC

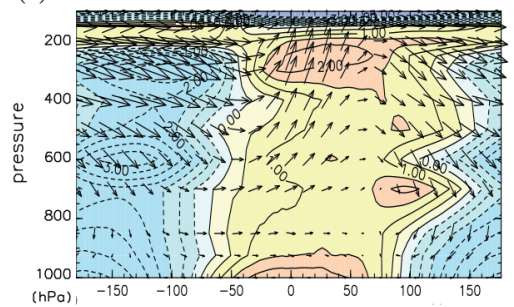

(1) $\mathrm{MIT}$

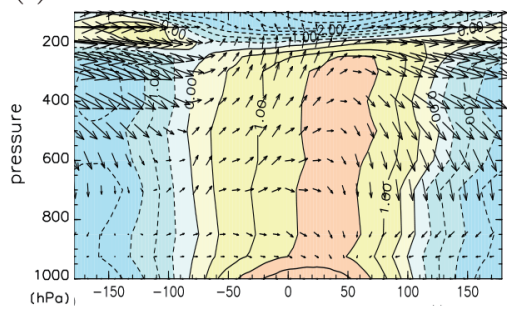

(o) UKMOn96

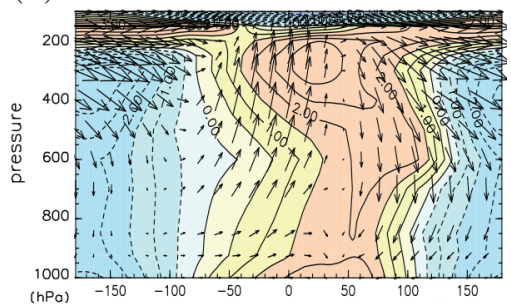

$0.4 \mathrm{~Pa} / \mathrm{s}$

$60 \mathrm{~m} / \mathrm{s}$

Fig. 25. Vertical distributions of time mean anomalies of temperature, zonal velocity, and p-velocity along the equator in the $3 \mathrm{KW} 1$ runs from the $15 \mathrm{APE}$ models. Contour interval and magnitudes of wind vector components are indicated at the bottom. 
vertical motion, and latent heating are all dominated by the zonal wavenumber one variations in all of the models, although their vertical structures vary considerably.

The longitudinal distributions of the upward motion and positive heating anomalies roughly coincide with the distribution of the precipitation anomalies (the left column of Fig. 20) in most of the models The anomaly of vertical motion in CSIROstd is an exception; downward motion dominates over most of the lower troposphere below $600 \mathrm{hPa}$. This is presumably the result of the distinct double-peak structure of the positive precipitation anomaly in CSIROstd around the warm SSTA (Fig. 19c) and the associated trough line of the precipitation anomaly along the equator.

The location of the temperature anomalies (represented by the contours in Fig. 25) shifts to the east of the precipitation anomalies, typically by $20^{\circ}$ to $40^{\circ}$. As for the temperature anomaly in the lower troposphere, the eastward shift may be related to the advection of colder air from the higher latitudes by the meridionally converging low-level wind anomaly that develops between $-60^{\circ}$ and $0^{\circ}$ longitude (Fig. 22). As for the temperature anomaly in the middle and upper troposphere, the eastward shift is presumably caused by eastward advection by the westerly wind invading the tropics (Fig. 2b). The vertical structure of the temperature anomaly is not simple and model dependent. However, several common characteristics can be identified, one of which is that the temperature anomalies in the middle troposphere are weaker, and shifted to the east of the precipitation anomalies. In AGU, GSFC, and NCAR, the temperature anomalies around the 600 $\mathrm{hPa}$ level are more complicated, and we find shallow, eastward shifted, positive anomalies, presumably related to the melting and/or freezing of hydrometeors.

Compared with the temperature anomalies at the equator in 3KEQ (Fig. 11), the temperature anomalies at the equator in $3 \mathrm{KW} 1$ are considerably stronger, and the typical temperature increase in the positive anomalies at $700 \mathrm{hPa}$ is $1.5-2 \mathrm{~K}$ in $3 \mathrm{KW} 1$, whereas it is $0.5-1$ $\mathrm{K}$ in $3 \mathrm{KEQ}$. In other words, the weak temperature gradient approximation (Sobel et al. 2001) does not apply well in $3 \mathrm{KW} 1$, even along the equator.

The heating anomaly corresponding to the positive precipitation anomaly is intense in the upper troposphere for all of the models except for LASG. Accordingly, the upward motion around the peak of the precipitation anomaly is stronger in the upper troposphere than in the lower troposphere in most of the models. However, again as in $3 \mathrm{KEQ}$, the correspondence between the vertical structures of heating and vertical motion is not perfect; for example, the thin heating anomaly just above $600 \mathrm{hPa}$, and the thin cooling anomaly just below in AGU (Fig. 27a), are not reflected in the vertical velocity response. Instead, a shallow warm anomaly develops at $600 \mathrm{hPa}$ to the east of the precipitation anomaly (Fig. 25a). The shallow regions of cooling, found in ECMWF07, K1JAPAN, and NCAR, and induced by the evaporation of rain in the lowest atmosphere are not reflected in the corresponding vertical velocity anomalies. This is presumably due to the effects of other physical processes that offset the cooling, such as turbulent mixing, and also its proximity to the sea surface, which prohibits the vertical motion.

The signature of the heating anomalies in the regions of suppressed precipitation is mostly negative (Fig. 26). The vertical structures of the heating anomalies in the regions of the positive and negative precipitation anomalies differ. In most of the models, heating in the positive precipitation anomaly is more intense and located at higher levels, and is richer in the components with short vertical wavelengths. This deviation from the perfect anti-symmetry between the positive and negative heating anomalies is also reflected in the structure of vertical motion in the corresponding model (Fig. 25). K1JAPAN exhibits an additional interesting characteristic: outside the region of positive rainfall anomaly, a shallow, but significant, positive heating anomaly develops below $800 \mathrm{hPa}$ (Fig. 26f) between $\lambda=50^{\circ}$ and $180^{\circ}$. A corresponding shallow upward motion anomaly also exists (Fig. 25j). The origin of this peculiar behavior has not been identified.

In most of the models, the westerly wind anomaly over the longitudes of the cold SSTA, which was mentioned as a characteristic of the $250 \mathrm{hPa}$ level in Fig. 21, extends over the upper half of the troposphere, and contributes to the zonal mean zonal wind anomaly, which will be discussed later.

\subsection{Relationships among the variables}

As was done for $3 \mathrm{KEQ}$, we will now examine the relationships among the responses of different variables and try to identify the causes of this variation in the comparisons among the APE models. We will again use the peak-to-peak range as the gross measure of the amplitude of the anomaly of a variable.

\section{a. Dynamical response}

Figure 28a shows the scatter plot of the amplitude of the precipitation anomaly averaged over the equatorial latitudes between $\pm 15^{\circ}$ and the amplitude of the geopotential anomaly at the $250 \mathrm{hPa}$ level at the equator. 


\section{KW1 Anomalies of T Tendency by Cloud at the Equator}

(a) AGU ci:4E-5 $\mathrm{K} \mathrm{s}^{-1}$

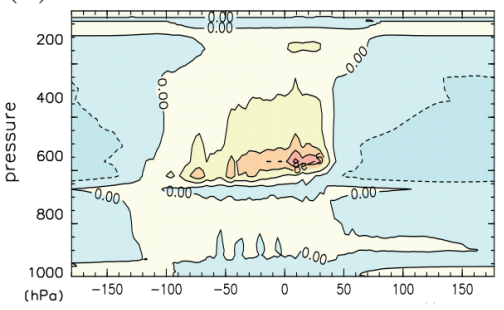

(d) ECMWF07 ci:2E-5 $\mathrm{K} \mathrm{s}^{-1}$

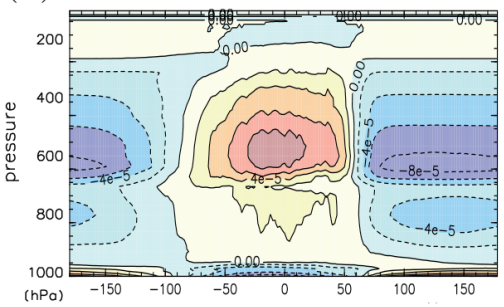

(g) LASG ci:2E-5 $\mathrm{K} \mathrm{s}^{-1}$

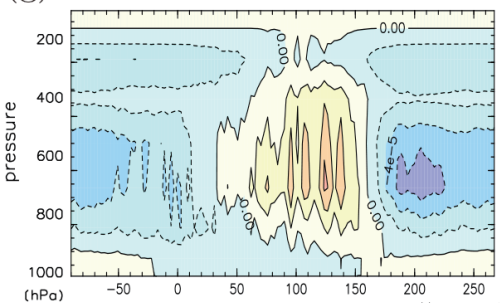

(b) DWD ci:4E-5 $\mathrm{K} \mathrm{s}^{-1}$

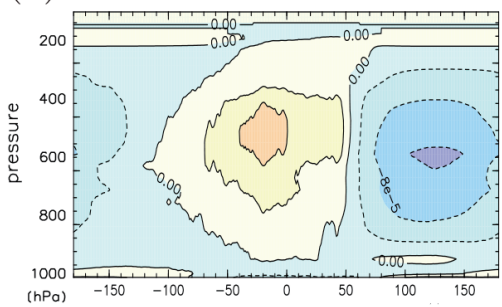

(e) GSFC ci:2E-5 $\mathrm{K} \mathrm{s}^{-1}$

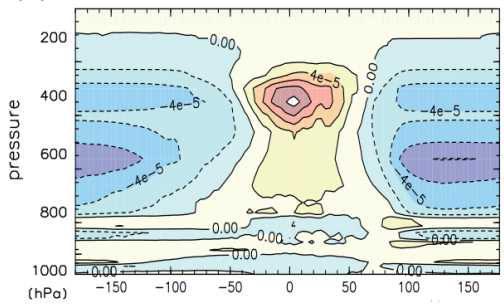

(h) NCAR ci:1E-5 $\mathrm{K} \mathrm{s}^{-1}$

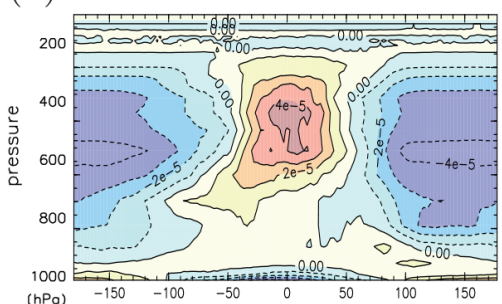

(c) ECMWF05 ci: $5 \mathrm{E}-5 \mathrm{~K} \mathrm{~s}^{-1}$

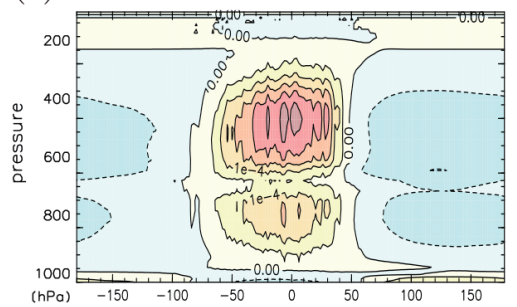

(f) K1JAPAN ci:1E-5 $\mathrm{K} \mathrm{s}^{-1}$

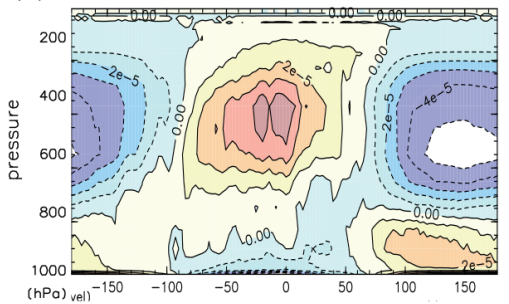

(i) UKMO ci: $4 \mathrm{E}-5 \mathrm{~K} \mathrm{~s}^{-1}$

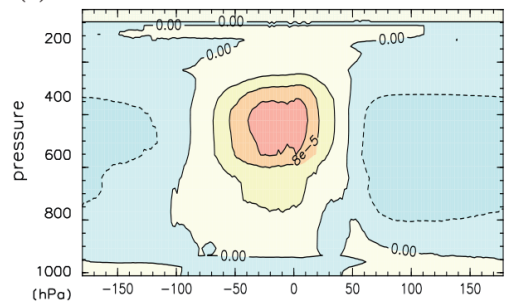

Fig. 26. Vertical distributions of time mean temperature tendencies at the equator in the $3 \mathrm{KW} 1$ runs from the nine APE models. The sum of parameterized and resolved tendensies are plotted. Contour interval is indicated at the top of each panel. Vertical axis is pressure.

The amplitude of precipitation is shown in units of the equivalent amount of latent heat; i.e., $1000 \mathrm{~W} \mathrm{~m}^{-2}$ corresponds to $4 \times 10^{-4} \mathrm{~kg} \mathrm{~s}^{-1} \mathrm{~m}^{-2}$. It can be seen that the range of scattering of the geopotential anomalies is comparable to the typical magnitude of geopotential variation (half of the peak-to peak magnitude of the anomaly), but that no correlation with the precipitation anomaly is evident. Such characteristics are also seen in the geopotential anomalies at other latitudes (not shown). Other factors, such as the difference in the vertical structure of heating may explain the scattering.

Figure 28b shows the relationship between the upper tropospheric meridional transport of zonal momentum by the stationary eddy at $10^{\circ} \mathrm{N}$, and the amplitude of the zonal mean zonal wind anomaly at $200 \mathrm{hPa}$ averaged within $\pm 10^{\circ}$ of latitude. Some correspondence between the two variables is seen, suggesting the importance of the stationary eddy in the zonal mean wind acceleration. However, the correlation is not very significant; for example, the difference between the magnitudes of momentum flux in DWD and NCAR is only modest, but the zonal mean zonal wind acceleration differs by a factor of two. Figure 28c shows a scatter plot of precipitation in $3 \mathrm{KW} 1$ within the $\pm 10^{\circ}$ latitude band and the amplitude of the zonal mean zonal wind anomaly at 200 $\mathrm{hPa}$, where a weak negative correlation can be noted. The positive correlation with the poleward export of easterly momentum by the stationary eddy (Fig. 28b), and the negative correlation with equatorial precipitation (Fig. 28c), are consistent with the results of Kraucunas and Hartmann (2005), who compared idealized GCM experiments with prescribed zonally symmetric and zonally varying heating to show that equatorial superrotation is induced by the export of easterly momentum by a tropical stationary eddy, but decelerated by the vertical advection of easterly momentum by upward motion near the equator. 


\section{KW1 Anomalies of T Tendency at the Precipitation Maximum}

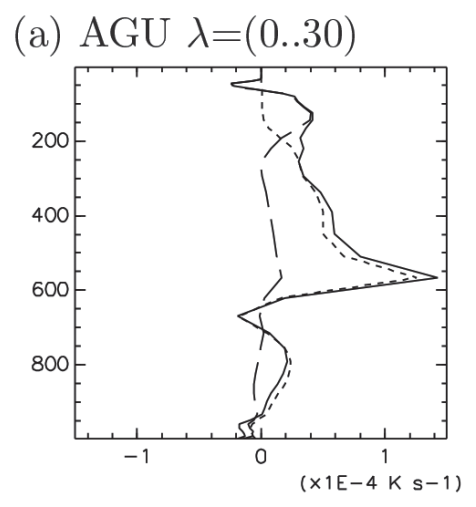

(d) ECMWF07 $\lambda=(-20 . .0)$

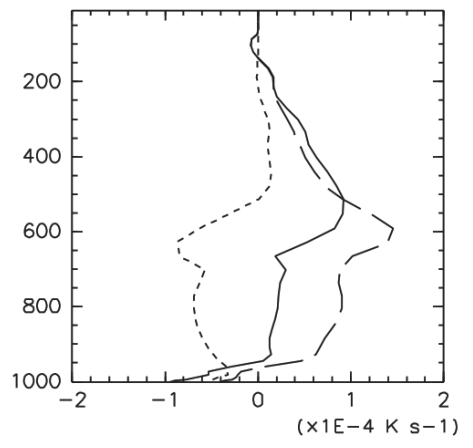

(g) LASG $\lambda=(10 . .50)$

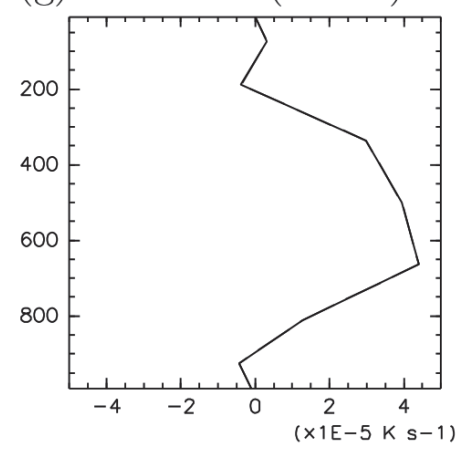

(b) DWD $\lambda=(-30 . .0)$

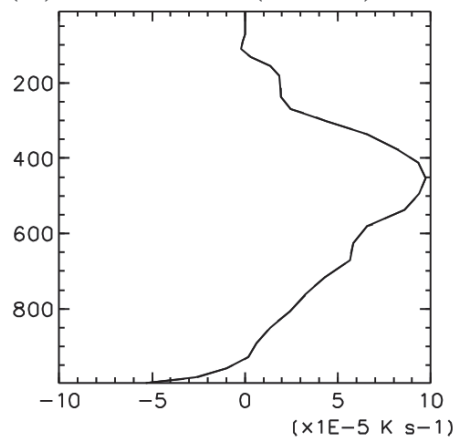

(e) GSFC $\lambda=(0 . .20)$

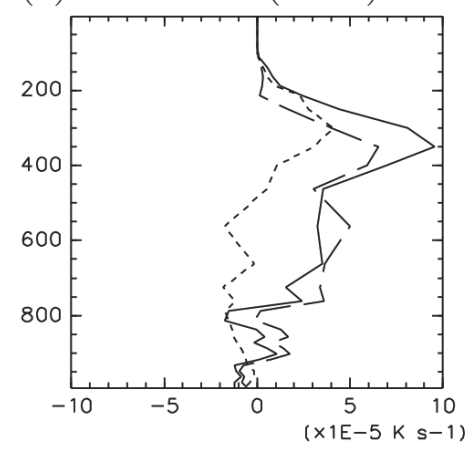

(h) NCAR $\lambda=(0 . .20)$

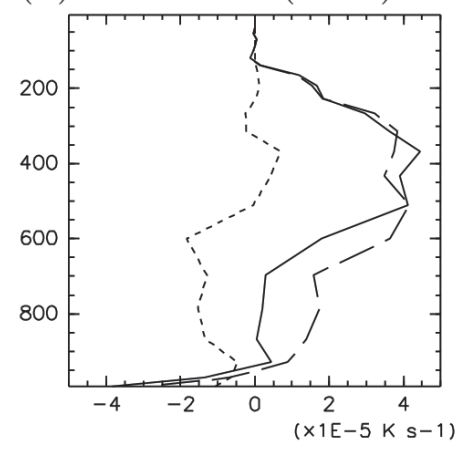

(c)ECMWF05 $\lambda=(-20 . .0)$

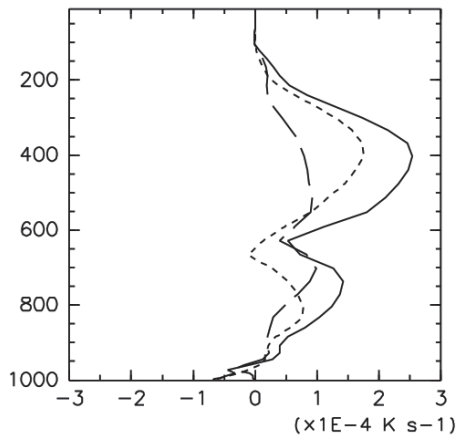

(f) K1JAPAN $\lambda=(-20 . .0)$

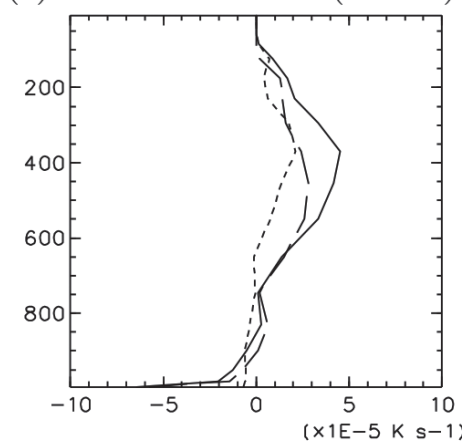

(i) UKMO $\lambda=(-30 . .0)$

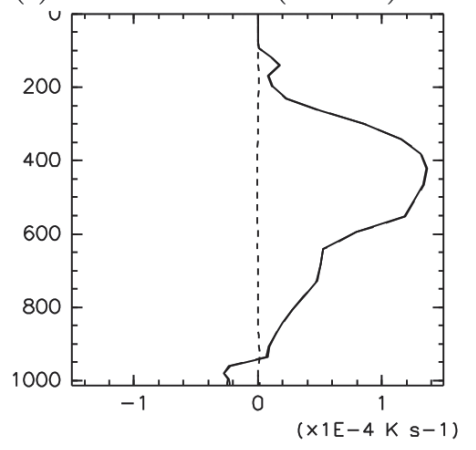

Fig. 27. Vertical profiles of time mean temperature tendencies at the SST anomaly in the $3 \mathrm{KW} 1$ runs from the nine APE models for which data are available. Units: $\mathrm{K} \mathrm{s}^{-1}$. Vertical axis is pressure. Dotted and dashed lines indicate tendencies due to resolved clouds and parameterized convection, respectively, and solid line indicates the sum of the two. Note that heating due to resolved clouds is not available for DWD and LASG.

\section{b. Factors controlling the precipitation anomaly}

Like we did for 3KEQ, we now examine the response of several variables that may influence the precipitation anomaly. First, we compare the amplitude of the precipitation anomaly with that of the low-level temperature anomaly, which may affect the degree of convective instability. Second, we compare it with the zonal mean intensity of precipitation in the CONTROL experiment, which we take to represent the basic state of precipitation. Third, we compare it with the amplitude of the evaporation anomaly, which contributes to the supply of moisture for the enhanced precipitation. As in Fig. 15, we use the following two values for the amplitude of the anomalies: one is the intensity averaged within $\pm 5^{\circ}$, which reflects the variety of the meridional structure among the models, and the other 
(a)

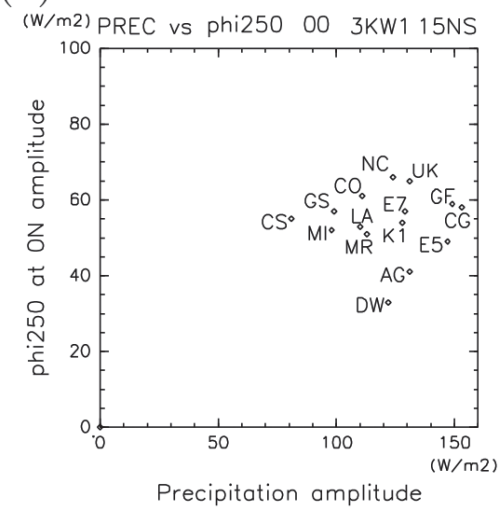

(b)

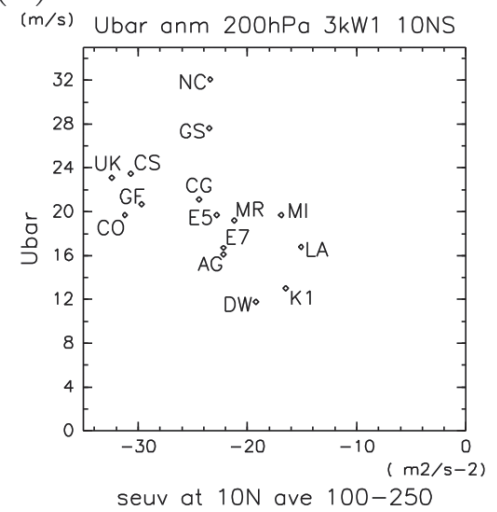

(c)

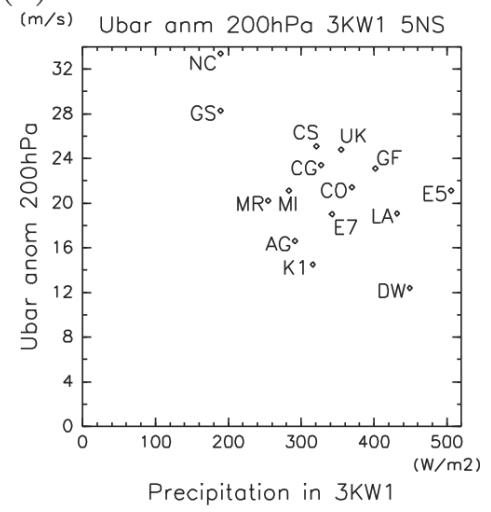

Fig. 28. Scatter plots showing the dynamical response of the variables in the $3 \mathrm{KW} 1$ runs from the $15 \mathrm{APE}$ models. (a) The amplitude of the wavenumber one component of geopotential anomaly at $250 \mathrm{hPa}$ at the equator vs that precipitation anomaly averaged within $15^{\circ}$ of the equator. (b) Poleward zonal momentum flux associated with the stationary eddy at $10^{\circ} \mathrm{N}$ averaged for pressure levels between 100 and $250 \mathrm{hPa}$ vs the zonal mean acceleration averaged within $10^{\circ}$ of the equator at $200 \mathrm{hPa}$. (c) Zonally mean precipitation averaged within $5^{\circ}$ of the equator vs zonal mean acceleration averaged within $15^{\circ}$ of the equator at $200 \mathrm{hPa}$. See Table 1 for the label legends.

is the intensity averaged within $\pm 15^{\circ}$, which indicates the longitudinal variation of the precipitation anomaly over the ITCZ as a whole. ECMWF05 and ECMWF07 are excluded from the comparison of the precipitation anomaly averaged within $\pm 5^{\circ}$. However, we include these two models in the comparison of the precipitation anomaly averaged within $\pm 15^{\circ}$ because the intensity of the precipitation response averaged within this latitudinal band does not appear to be seriously affected by the narrow meridional scale of the SSTA setup of these two runs, as seen in the central column of Fig. 20.

Figure 29a is a scatter plot of the amplitude of the precipitation anomaly against the amplitude of the temperature anomaly at $925 \mathrm{hPa}$ averaged within the $\pm 5^{\circ}$ latitude band. The amplitude of the temperature anomaly varies by a factor of 1.5 among the models. The range is considerably narrower than that for 3KEQ (Fig. 15a). Figure 29d is a similar scatter plot, but for the average within the $\pm 15^{\circ}$ latitude band. The amplitude of the temperature anomaly varies by a factor of two among the models. In contrast to 3KEQ (Figs. 15a,d), no trace of a positive correlation can be found between the amplitudes of the precipitation anomaly and the low-level temperature anomaly. This absence of correlation arises from the intense temperature anomaly that occupies the whole depth of the troposphere (Fig. 26), which is roughly in phase with the low-level temperature anomaly and so tends to counterbalance the variation in convective instability caused by the low-level temperature and moisture anomalies.

Figure 29b is a scatter plot of the amplitude of the precipitation anomaly against the amplitude of the surface latent heat flux anomaly averaged within the $\pm 5^{\circ}$ latitude band. As in the case of $3 \mathrm{KEQ}$ (Fig. 15b), the amplitude of the latent heat flux anomaly varies by a factor of three. However, the correlation between the amplitude of the precipitation anomaly and the amplitude of the surface latent heat flux anomaly is very weak. Figure $29 \mathrm{e}$ is a similar scatter plot, but for the averages within the $\pm 15^{\circ}$ latitude band. The scattering of the amplitude of the latent heat flux anomaly is narrower than that for the $\pm 5^{\circ}$ latitude band, and varies by a factor of about two. As in the case of 3KEQ, there is a good degree of correlation between the two amplitudes. Moreover, the ratio of the amplitude of the latent heat anomaly to the amplitude of the precipitation anomaly is larger than for $3 \mathrm{KEQ}$ and reaches about $40 \%$, compared with about $25 \%$ for 3KEQ (Fig. 15e).

Figure $29 \mathrm{c}$ is a scatter plot of the amplitude of the precipitation anomaly against the zonal mean precipitation amount in CONTROL averaged within the $\pm 5^{\circ}$ latitude band. As in $3 \mathrm{KEQ}$, a positive correlation is seen, and this seems to be reasonable because, as is pointed out in Section 5.2a, the response of precipitation near the equator exhibits a large model dependence whose latitudinal structure is inherited from the corresponding CONTROL run of the model. Figure $29 \mathrm{f}$ is a similar scatter plot, but for the average within the $\pm 15^{\circ}$ latitude band. Scattering among the models is 
(a)

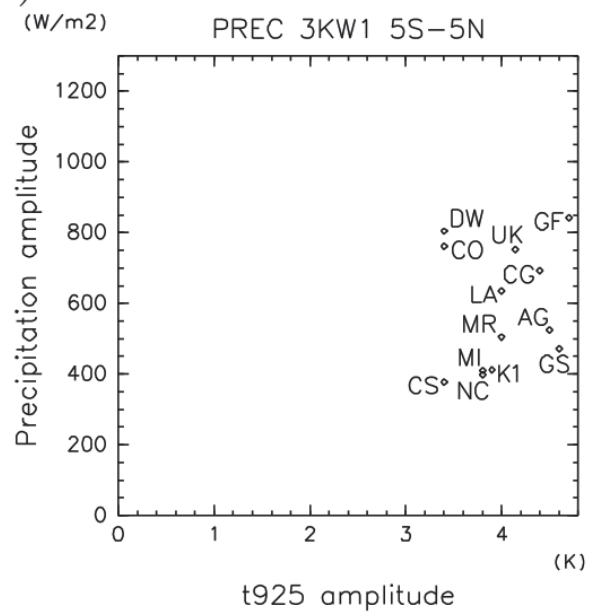

(b)

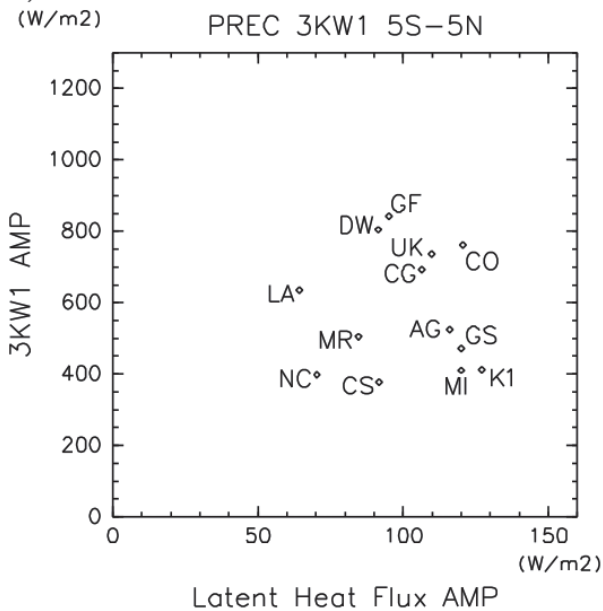

(c)

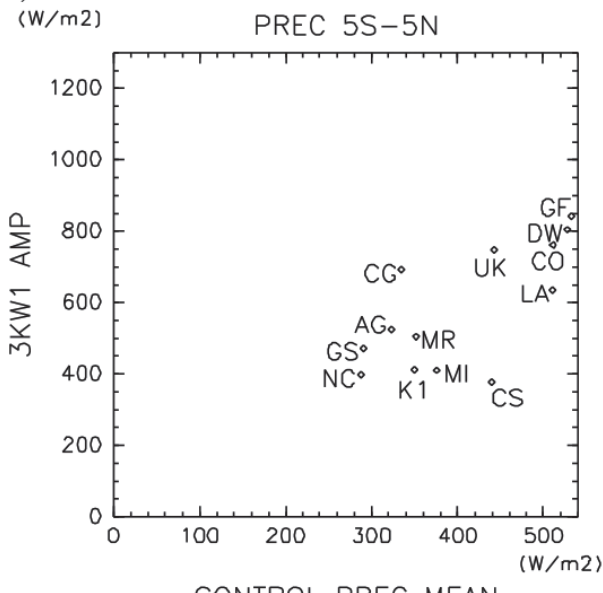

(d)

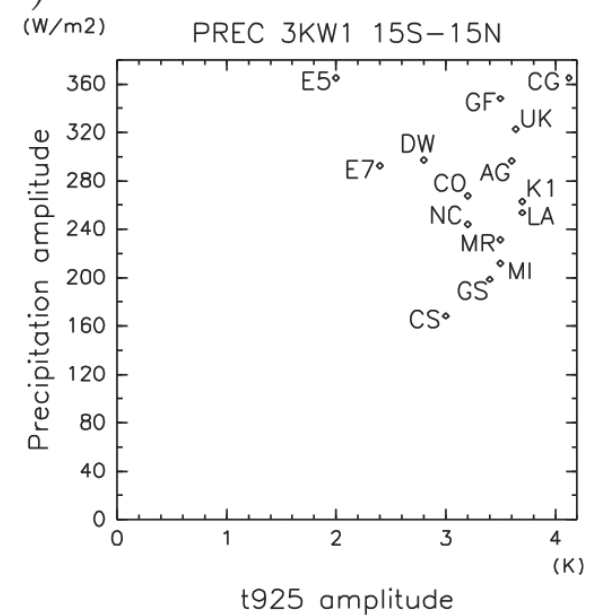

(e)

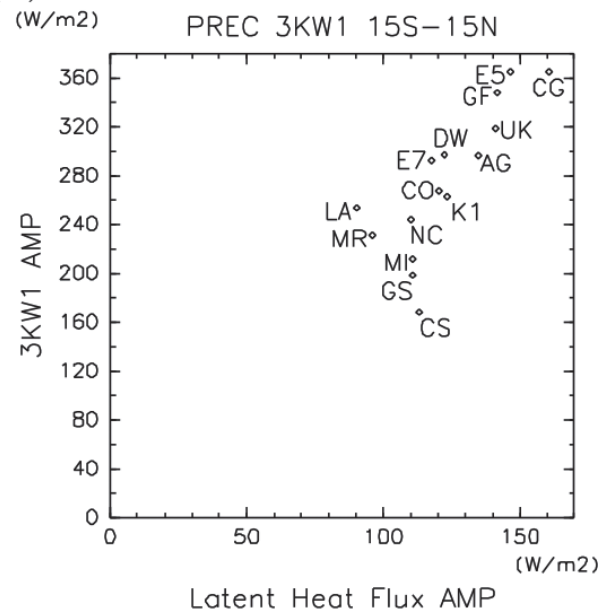

(f)

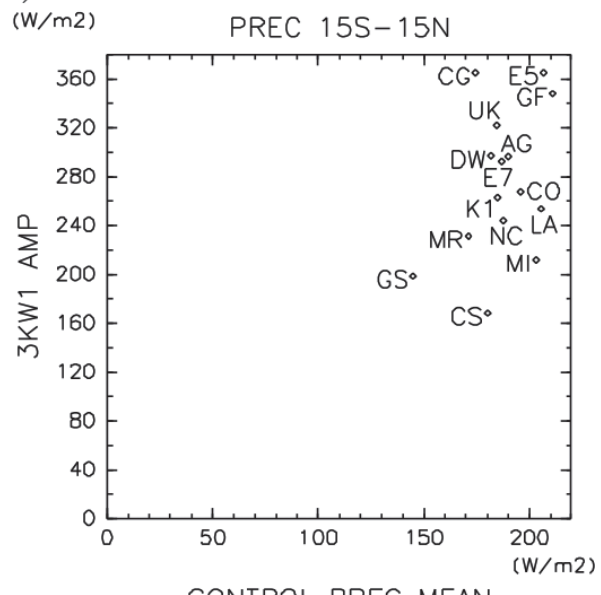

Fig. 29. As Fig. 15 but for 3KW1. 
(a)

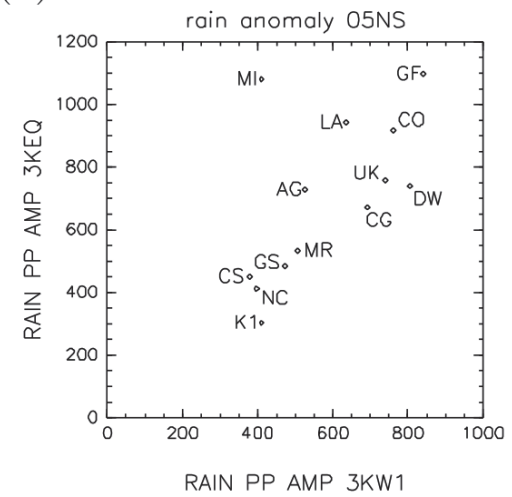

(d)

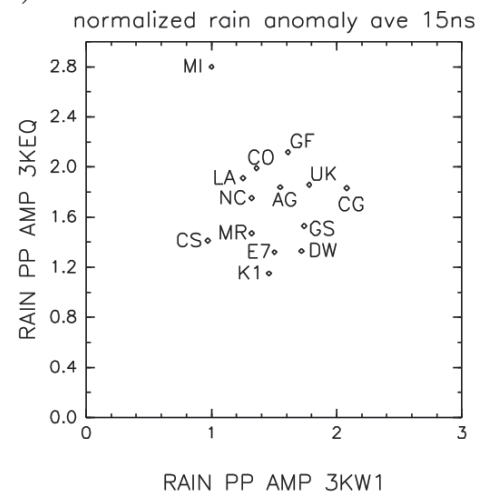

(b)

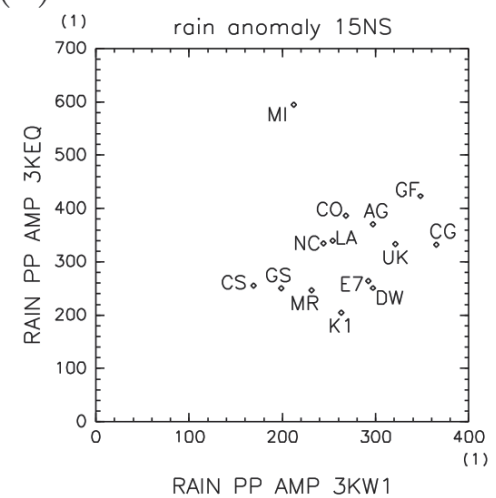

(e)

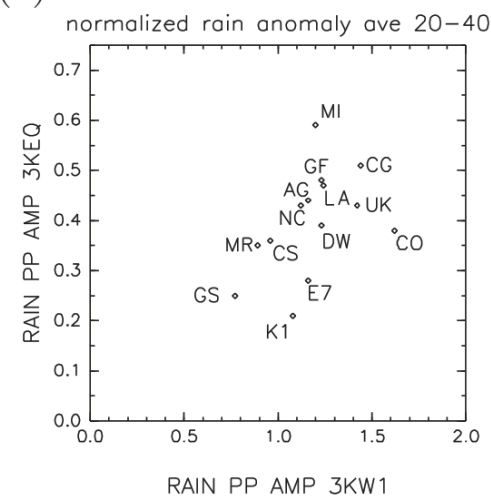

(c)

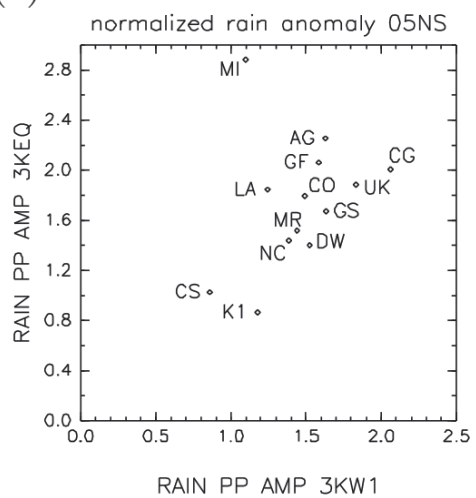

(f)

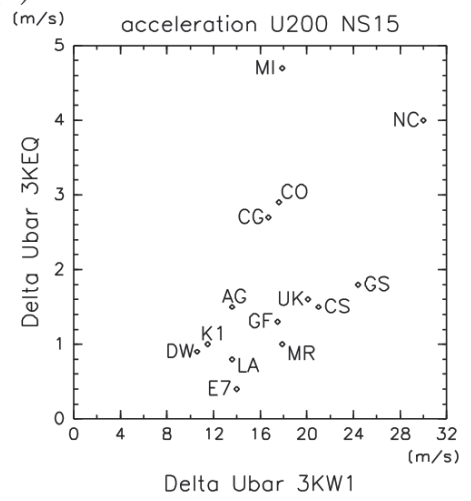

Fig. 30. Scatter plots comparing the amplitudes of anomalies in the 3KEQ and 3KW1 runs of the APE models. (a) Precipitation averaged over the equatorial latitudinal band within $\pm 5^{\circ}$. (b) As (a) but averaged within $\pm 15^{\circ}$. (c) As (a) but for the amplitude of the precipitation anomaly normalized by the time mean zonal mean precipitation of the corresponding CONTROL run. (d) As (c) but averaged within $\pm 15^{\circ}$. (e) As (c) but averaged over the latitudinal band from $20^{\circ} \mathrm{N}$ to $40^{\circ} \mathrm{N}$. (f) Change of zonal mean zonal wind at $200 \mathrm{hPa}$ averaged over the equatorial latitudinal band within $\pm 5^{\circ}$. See Table 1 for the label legends.

reduced because the meridional structure of the ITCZ is averaged out. As in 3KEQ, a weak positive correlation is seen.

\subsection{Comparison between the responses in $3 K E Q$ and $3 K W 1$}

So far, we have described the variety of behavior in the APE models for 3KEQ and 3KW1 separately. The final aspect of the model comparisons in the present paper briefly compares the responses to 3KEQ and $3 \mathrm{KW} 1$, but we limit ourselves mostly to precipitation, which is the primary forcing agent in the atmospheric general circulation, and might be expected to respond more directly than other variables to a given SSTA. The response of other variables would be complicated by a number of interacting processes within the models.

Figure 30a shows the relationship between the amplitudes of the precipitation anomalies of 3KEQ and $3 \mathrm{KW} 1$ averaged within $\pm 5^{\circ}$ of latitude among the models. The anomalies associated with $3 \mathrm{KEQ}$ and $3 \mathrm{KW} 1$ are roughly in proportion to each other. If we consider MIT to be an outlier and disregard it, the extent of the correlation increases further. A similar tendency can also be found for precipitation averaged within $\pm 15^{\circ}$ of latitude (Fig. 30b).

The good correspondence between the intensities of the precipitation anomalies in the 3KEQ and $3 \mathrm{KW} 1$ settings is, in fact, not surprising, because both are correlated with the precipitation intensity of the corresponding CONTROL run (Figs. 15c,d, and $28 \mathrm{a}, \mathrm{b})$. Even normalized by the precipitation in the corresponding CONTROL run, some correlation still remains between the precipitation anomalies averaged within $\pm 5^{\circ}$ of latitude (Fig. 30c). Some positive 
correlation can also be noted between the normalized mid-latitude responses in 3KEQ and 3KW1 (Fig. 30e). However, in the normalized anomalies averaged over $\pm 15^{\circ}$ of latitude, we cannot find any correlation (Fig. $30 \mathrm{~d})$. The origin of these characteristics is unclear, and we leave its pursuit for a future study.

Figure 30f shows the correspondence between the amplitudes of the zonal mean zonal wind anomalies in 3KEQ and 3KW1. Although uncertainty of about $1 \mathrm{~m} \mathrm{~s}^{-1}$ should be incorporated into the anomalies for 3KEQ as noted in Section 4, some degree of positive correlation can be identified.

Finally, we comment on the vertical structures of the heating response, which are shown in Figs. 12 and 13 for 3KEQ, and Figs. 26 and 27 for $3 \mathrm{KW} 1$, respectively. Comparing the heating structures in each of the models, we find that, in general, the peak intensity of the positive heating anomaly in 3KEQ is stronger than that in $3 \mathrm{KW} 1 \mathrm{in}$ most of the models. This difference reflects the difference between the intensities of the positive precipitation anomalies in 3KEQ and 3KW1 (see Figs 5 and 20). We also see the vertical structures of heating in the two SST settings are roughly similar in most of the models. Some differences do exist, including the heating profile in LASG being vertically two-peaked for 3KEQ but one-peaked for 3KW1, and heating in the lower troposphere in ECMWF05 being more intense for $3 \mathrm{KW} 1$ than $3 \mathrm{KEQ}$.

\section{Summary and remarks}

Varieties in the precipitation and circulation structures that appear in response to a localized (3KEQ and 1 KEQ) and a planetary-scale SSTA (3KW1) superimposed on a zonally homogeneous SST (CONTROL) in the 15 AGCMs participating in the APE have been described and compared. We have examined only the time mean response defined as the difference in the temporal average of the atmospheric state for an SSTA to that of the corresponding CONTROL run.

\subsection{Characteristics of the response to the SSTAs in the APE}

a. The response to a localized SSTA

Gross features of the anomalies that appear in all of the models in response to the localized equatorial warm SSTA found in the 3KEQ experiment can be summarized as follows. (i) A distinct positive precipitation anomaly, whose amplitude exceeds twice the mean precipitation at the equator in the corresponding CONTROL run, develops over the SSTA. On the other hand, a weak but widespread negative anomaly appears in the ITCZ outside the SSTA. Corresponding to the positive precipitation anomaly, a positive heating anomaly develops over the SSTA, and is typically found in the upper half of the troposphere in most of the models. (ii) The divergent flow from the heating anomaly forces a pair of intense upper tropospheric anticyclones at subtropical latitudes to the north and south of the precipitation anomaly. Influenced by the strong westerly jets invading to latitudes near the equator, the anticyclones extend eastward. (iii) Disturbed by the flow associated with the anticyclones, the Kelvin wave response expected to the east of the positive precipitation anomaly is almost completely obscured. The baroclinic equatorial Rossby wave response expected to the west is also weak or absent, presumably because of the very small value of absolute vorticity contributed by the anticyclonic shear on the equatorward sides of the westerly jets mentioned above. As a result, the appearance of the tropical response structure is very different from the structure that characterize the standard framework of the thermal response problem of Matsuno (1966) and Gill (1980). (iv) From the off-equatorial anticyclonic anomalies at the longitudes of the SSTA, equivalent barotropic Rossby wavetrains are emitted and propagate poleward, but are immediately refracted back to the tropical latitudes at around $10,000 \mathrm{~km}$ east of the SSTA, resulting in a distinct deep warm signal in the tropics that is partially separated from the warm region over the SSTA. The Rossby wavetrains further propagate eastward along the waveguides associated with the mid-latitude westerly jets. (v) The mid-latitude dynamical response described above induces nonnegligible precipitation anomalies, mainly on the equatorward flanks of the westerly jets, which are composed of the negative anomalies around the longitudes of the SSTA, positive anomalies to the east between latitudes of $50^{\circ}$ and $100^{\circ}$, and slight enhancement of the wavenumber 5 quasi-stationary features identified in the CONTROL experiment.

The variety of the responses found among the models can be summarized as follows. (i) The intensity, structure, and location of each element of the responses summarized above are considerably model dependent. The peak-to-peak amplitude of the precipitation anomaly at the equator varies by more than a factor of three, reflecting the variety of the meridional structure of the anomalies, each of which is basically inherited from the meridional structure of the ITCZ precipitation of the corresponding CONTROL run. The variety in the amplitudes of the responses is reduceds when they are averaged meridionally across the $\pm 15^{\circ}$ latitude band centered on the equator that covers all of the equatorial precipitation anomaly, but amplitude still 
varies by more than a factor of two among the models. (ii) The vertical structures of the heating anomalies differ among the models, presumably reflecting the characters of particular convective cloud parameterizations used in the different models. (iii) The details in the structures of the precipitation anomalies over the equatorial region vary considerably, although they share the dissimilarity to the standard Matsuno-Gill pattern as mentioned previously. (iv) The intensities of the Rossby wavetrains vary by more than a factor of two, and to some extent vary according to the intensities of the precipitation anomalies averaged within $\pm 15^{\circ}$ of the equator, suggesting that the Rossby waves are essentially a linear response to the heating anomaly over the SSTA. (v) The factors that control the intensity of the precipitation anomaly in different models were sought, but were not successfully identified.

The intensities of the anomalies associated with the stronger localized equatorial SSTA (3KEQ), and those with the weaker SSTA (1KEQ) of the same shape in the corresponding models were compared. The results indicate that the intensity of the precipitation anomaly over the SSTA varies roughly in proportion to the intensity of the SSTA in each of the models. The intensity of the Rossby wavetrain also increases in each model as the intensity of the SSTA increases, but proportionality does not necessarily hold.

\section{b. The response to the zonal wavenumber one SSTA}

Gross features of the anomalies that appear in all of the models in response to the wavenumber one variation of the equatorial SSTA found in the 3KW1 experiment are summarized below. (i) Both the precipitation and dynamical responses are characterized by the wavenumber one zonal variation. (ii) In the warm (cold) SST region, a zonally extensive positive (negative) precipitation anomaly appears. The amplitude of the anomaly is comparable with, or more than, the zonal mean precipitation amount in the corresponding CONTROL run. (iii) The vertical structure of the heating anomaly is strongly weighted to the upper troposphere in most of the models. (iv) In the upper troposphere, affected by the divergent wind from the precipitation anomaly, a region of small absolute vorticity extends poleward, whose longitudinal location shifts eastward from the precipitation anomaly. In contrast, in the cold SST region, the region of small absolute vorticity almost disappears, and the subtropical westerly jets become weaker, but more widespread, invading deep into the tropics and almost to the equator. In the lower troposphere, a low pressure anomaly develops to the east of the positive precipitation anomaly. (v) The upper tropospheric wind response at the equatorial precipitation maximum is dominated by zonal convergence and meridional divergence. At low levels, convergence is dominated by the meridional component. These characteristics are, as in the response to the localized SSTA, distinctly different from those of the standard thermal response of the Matsuno-Gill pattern. (vi) Dynamical responses at lower and higher latitudes exhibit a contrasting vertical structure. Equatorward of the subtropical westerly jets, the response is baroclinic; in the warm SST region, the upper (lower) level response is high (low) pressure, whereas in the cold SST region, the upper (lower) level response is low (high) pressure. Poleward of the westerly jets, the response is barotropic; the low (high) pressure response dominates in the longitudes of warm (cold) SST, both in the upper and lower troposphere. (vii) Associated with the mid-latitude dynamical response, variations of precipitation develop in the baroclinic zones, whose amplitude are about half of the zonal mean precipitation in the corresponding CONTROL run. (viii) Considerable westerly acceleration of the zonal mean zonal wind occurs in the upper troposphere around the equator.

Less variety was found in the response to the wavenumber one SST variation compared with the large variety found in the response to a localized SSTA. The variety of the responses noted among the models are summarized below. (i) The intensities of the precipitation anomalies near the equator vary by a factor of almost five, and the meridional distributions of the anomalies mostly reflects the structure of the ITCZ in the corresponding CONTROL run. The shape and longitudinal phase of the precipitation anomalies vary significantly. The variation in the amplitudes is considerably reduced when the anomalies are averaged meridionally over a band within $\pm 15^{\circ}$ of the equator that covers all of the equatorial precipitation anomalies, and the peak-to-peak amplitudes are typically $150 \%$ of the corresponding amounts in CONTROL. (ii) The intensity of the geopotential height anomalies over the subtropics and high latitudes varies by a factor of two among the models. Although a similar range of variability exists in the amplitude of the precipitation anomaly, no clear relationship is evident between the precipitation and dynamical response amplitudes. (iii) The anomalies of zonal mean zonal wind in the upper troposphere vary by a factor of three, and can be related positively to the zonal momentum transport by a stationary wave, and negatively related to the mean equatorial precipitation intensity.

Comparing the responses of the participating models to the 3KEQ SSTA with those to the 3KW1 SSTA, 
we saw that each model responds to both SSTAs in a consistent manner. For example, the meridional structures of the equatorial precipitation anomalies are similar, and the models with a relatively intense precipitation response to 3KEQ also tend to exhibit a more intense precipitation response to $3 \mathrm{KW} 1$ than the other models.

\subsection{Comments on the observed response to SSTA}

The intensity and horizontal extent of the SSTA in 3KEQ are not very different to those of the SSTA in the warm phase of ENSO, and those of 3KW1 are somewhat similar to those associated with climatological zonal variation of tropical SST over the Indian and Pacific oceans, so that it may be appropriate to comment on the response to the SSTA observed in the real atmosphere.

During the warm phase of ENSO, the upper tropospheric geopotential anomaly is characterized by a pair of anticyclones in the subtropics at about $50^{\circ}$ longitude to the east of the peak equatorial SST anomaly, and a very weak Kelvin response, as depicted in Fig. 12a of Dima and Wallace (2007) for example (with sign reversed). These features are superficially similar to those established in the $3 \mathrm{KEQ}$ experiments. However, one should be cautious about the choice of a basic state against which an anomaly is defined. For example, as is shown in Fig. 2a of Dima and Wallace (2007), the climatology of upper tropospheric geopotential height is characterized by a pair of anticyclones around the longitude of the Maritime Continent and a pair of deep troughs in the eastern Pacific. On the other hand, as is shown Fig. 13a of the same reference, the geopotential field in the tropics is almost zonally symmetric during the warm phase of ENSO. As a result, the structure of the anomaly in the warm phase is, in fact, the structure of the climatology with the signature reversed; the dynamics shaping the response of geopotential to the SSTA should be interpreted, not with respect to the situation during the warm phase, but with regard to the situation of the climatology in mind. Of course, in the climatology, the pair of anticyclones are located to the west of the convection center. Another subtle feature of the response of the real atmosphere to the SSTA of ENSO, which is not necessarily independent from the issue above, is that the enhancement of convection in the equatorial central Pacific during El Nino is accompanied by suppression of convection in the western Pacific, presumably resulting from the cool SST anomaly (e.g., DeWeaver and Nigam 2004), which results in cancellation of the positive and negative Kelvin responses. On the other hand, each of the precipitation responses to the 3KEQ SSTA in the APE models is much closer to a monopole. In summary, the nature of the tropical response to 3KEQ in the present study should be regarded as being considerably different from the response structure that characterizes the anomaly during the warm phase of ENSO despite the certain superficial similarity between the two.

The structure of the response to $3 \mathrm{KW} 1$ is also very different from that of the Walker circulation in the real atmosphere. As can be seen in Fig. 4 of Dima and Wallace (2007), the observed Walker cell is characterized by a divergent zonal wind around the maximum of precipitation. In contrast, as described in Section 5, zonal wind is convergent at the precipitation maxima in $3 \mathrm{KW} 1$. By separating the horizontal wind field into rotational and divergent components (not presented here), we find that most of the zonal convergence/ divergence along the equator can be attributed to the rotational wind fields in both the climatological state and under $3 \mathrm{KW} 1$. In other words, the distinct difference between the observed Walker circulation and the response to $3 \mathrm{KW} 1$ originates mainly from the difference in the longitudinal phase of the Rossby response.

Bearing the considerable difference between the response to SSTA in 3KEQ and the anomaly associated with ENSO, and that between the response in $3 \mathrm{KW} 1$ and the observed Walker cell in mind, we consider that further quantitative comparison between the results obtained in this study and features observed in the real atmosphere is not appropriate.

\subsection{Remaining issues}

As is stated in Section 1, the focus of the present paper is to survey the results of AGCM experiments conducted with zonally varying SSTs in the APE, and a number of interesting issues found during the execution of the survey have been described, but are not pursued any further. These issues are summarized below.

As noted above, the structure of the response to the SSTA in the equatorial region is strongly affected by the intense upper tropospheric westerly wind due to the equatorward shift of the mid-latitude baroclinic jets in CONTROL compared with the real atmosphere (Section 3). It is implied that the characteristics of the response to the SSTA of the APE cannot necessarily be regarded as representative of those expected in realistic conditions. With this possibility in mind, it would be useful to conduct a small extension of the APE in which the same anomalies of SST are placed on a series of different zonally uniform basic state SSTs, e.g., the FLAT, QOBS, PEAKED, and CONTROL5N SST profiles defined in the specification of the APE 
(Blackburn and Hoskins, 2013). Such an extended series of experiments would strengthen the applicability of the SSTA response experiments of the APE to the real atmosphere. We conducted a preliminary study on such a series of experiments with one of the participating models of the APE, and have found that the responses to the 3KEQ SSTA are considerably different to those described here. These results will be reported elsewhere.

We have not examined the dynamical structure of the positive rainfall anomaly that develops over the localized SSTA in detail. Nor could we identify with any confidence the factors that determine the distribution and intensity of the precipitation anomaly in the models. Considering the diversity of the gross responses of precipitation and other variables (e.g., Figs. 15 and 16), understanding the issue of direct response requires more careful analyses of the parameterization tendency, not only that of cumulus parameterization, but also of boundary layer processes, radiation, etc. Even with such increasingly comprehensive datasets, feedbacks and interactions among various kinds of atmospheric processes would make development of an understanding of the issue a difficult task. For example, a preliminary survey of the time series of precipitation shows that the precipitation anomaly emerges as the increase in the activity of precipitating disturbances, as demonstrated in a previous study (Hosaka et al. 1998), suggesting that we must analyze not only the stationary features, but also transient components, if we are to understand the mechanism of the development of the precipitation anomaly over the SSTA. Models with a higher resolution (e.g., Yoshioka and Kurihara 2008) would also be informative.

We have pointed out that there are several types of precipitation anomalies outside the region of the SSTA, which are the negative anomaly along the ITCZ outside the SSTA in 3KEQ, the east-west dipoles at the equatorward flank of the baroclinic zones in 3KEQ and $3 \mathrm{KW} 1$, and the mid-latitude wavenumber 5 variation that also affects rainfall along the ITCZ. These are presumably indirectly induced as the remote dynamical responses forced by the precipitation anomaly over the SSTA. Examination of their generation mechanism, e.g., what kind of dynamical features are involved, how particular precipitation anomalies are induced, etc., is left for future research. This could also present a difficult problem due to the complex interaction among various processes in the model. One method that may be useful is to examine the time-dependent response (e.g., Jin and Hoskins 1995), namely, in an ensemble experiment (Toyoda et al. 1999; Nakajima et al. 2004).
More detailed analysis of wave propagation would also be useful using the wave activity diagnosis of Takaya and Nakamura (2001). Analysis of the transient disturbances mentioned earlier would also be useful for this purpose.

The mechanisms that produce the model dependence in the subtropical and extratropical responses to the SSTA are also left unresolved. It is probable that both the variety of the patterns and intensities of the heating anomalies above the SSTA, and the varieties in the structure of the zonal mean state of the atmosphere in different models contribute to the emergence of the varying response outside the tropics. As for the response to the localized SSTA (3KEQ), we have attempted a limited examination of the origin and propagation characteristics of the Rossby wavetrain in only one of the models (Appendix A). Such analysis must be repeated for the rest of the models to grasp the variety of behavior of waves among the models. As for the response to the wavenumber one SST variation (3KW1), preliminary analysis suggests that the influence of the eastward advection by a strong westerly wind, rather than westward propagation as a Rossby wave, is important. Further analysis of the modification of storm tracks by surface conditions, such as by Inatsu et al. (2002) or Sampe et al. (2013) should be applied to investigate the mechanism of the response. The effects of transient waves should also be investigated. These issues are also left for future studies.

We did not consider the properties and model dependence of transient disturbances in the presence of the SSTA. The space-time spectra of precipitation and outgoing longwave radiation presented in the APE-ATLAS (Williamson et al. 2012) exhibit both a large degree of change responding to the SSTA, namely $3 \mathrm{KW} 1$, and its considerable variety among the models, suggesting the presence and variety of the response of the transient disturbances. Analysis of composite structures of precipitation, such as carried out by Nakajima et al. (2013) from experiments with the CONTROL SST, would be useful to elucidate the response of transient disturbances. The analysis of these points would be worthwhile, particularly because the behavior of such disturbances may be important in shaping the stationary response structure. Unfortunately, the datasets required for such analysis are not collected in the SSTA experiments. Full analysis of transient disturbances awaits the next phase of the APE project, with its more complete collection and archiving of data. 


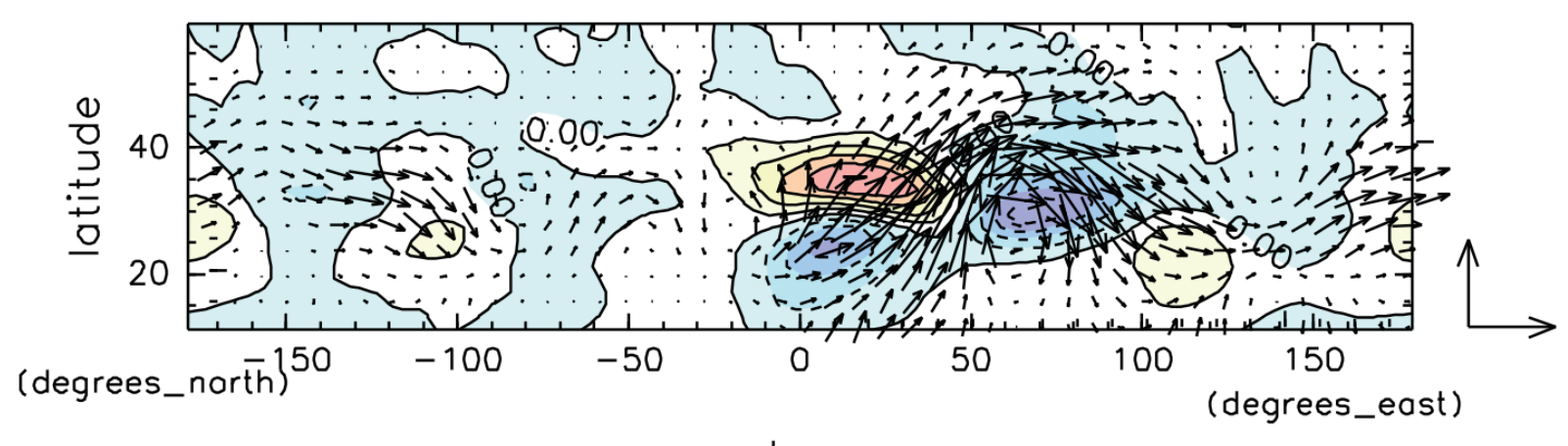

Ion

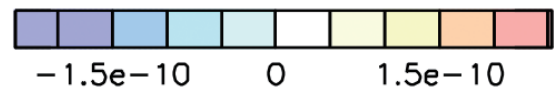

Fig. A1. Rossby wave source term of Sardeshmukh and Hoskins (1988), and the horizontal components of the wave activity flux vector of Takaya and Nakamura (2001) at $250 \mathrm{hPa}$ for the $3 \mathrm{KEQ}$ run of GFDL. Contour interval: $5 \times 10^{-11} \mathrm{~s}^{-2}$. Unit vectors correspond to $25 \mathrm{~m}^{2} \mathrm{~s}^{-2}$.

\section{Acknowledgements}

The authors sincerely thank two anonymous referees for carefully reading several versions of the manuscript. Their critical and constructive comments have helped the authors to greatly improve the original manuscript. Thoughtful advice from Hisashi Nakamura is also acknowledged. Stimulating and encouraging discussions with Drs. M. Blackburn and D. L. Williamson provided the great pabulum required to complete this work. Numerical integration of the AGU model was performed at the Earth Simulator Center, Japan Agency for Marine-Earth Science and Technology. Shin-ichi Takehiro helped the authors to prepare many of the figures. Analysis software and local computational environments were constructed using the resources of the GFD-DENNOU-CLUB, including GPhys, spmodel, and DCL. This work was supported by Grants-in-Aid (B) for Scientific Research (12440123 and 21340139).

\section{Appendix A \\ Behavior of Rossby waves in a 3KEQ experiment}

In this appendix, we briefly examine the behavior of stationary Rossby waves in the 3KEQ, and demonstrate that the upper-tropospheric low-latitude response that develops in response to the SSTA in 3KEQ has characteristics quite different from those of the thermal response problem of Matsuno (1966) and Gill (1980). We examine the 3KEQ GFDL model run as a specific example, but the results presented below can be applied to all of the APE models in a general sense.
The propagation characteristics of Rossby waves can be conveniently represented by the wave activity flux derived by Takaya and Nakamura (2001) in the quasi-geostrophic approximation, and the excitation of Rossby waves can be represented by the Rossby wave sources (or vorticity sources) defined by Sardeshmukh and Hoskins (1988), whose definition is summarized in Appendix B. Calculation of the wave activity flux requires the specification of a zonally uniform basic zonal flow. We employ the zonal and temporal mean zonal wind in the CONTROL run of the same model as the basic flow.

Figure A1 superimposes the horizontal component of the wave activity flux vector onto the contour diagram showing the distribution of the Rossby wave source at the $250 \mathrm{hPa}$ level. To save space, only the Northern Hemisphere is shown; the structure of the wave behavior is mostly symmetric about the equator (not shown here). Low latitude regions, where the flux cannot be suitably defined, are also omitted. We observe that a strong anticyclonic (negative) vorticity source exists around $(\lambda, \varphi)=\left(0^{\circ}, \pm 23^{\circ}\right)$, where the wave activity flux emerges and propagates northeastwards. The vorticity source consists mainly of the advective source (see Appendix B for the definition) that results from the meridionally directed divergent wind, whose origin is the precipitation anomaly above the SSTA, flowing on the steep gradient of absolute vorticity near the westerly jet. There are two additional areas of intense vorticity sources: one is the cyclonic (positive) vorticity source around $(\lambda, \varphi)=\left(15^{\circ}, \pm 35^{\circ}\right)$, and the other is the anti-cyclonic source around $(\lambda, \varphi)$ 
$=\left(70^{\circ}, \pm 32^{\circ}\right)$. These sources are mainly contributed by the divergent source related to the vertical motion associated with the wind flowing around the anticyclone centered at $(\lambda, \varphi) \sim\left(50^{\circ}, \pm 45^{\circ}\right)$ (see Fig. $6 \mathrm{~h}$ ) in the baroclinic zone, and should be interpreted as showing the vertical propagation of Rossby waves, rather than the "true sources". The vertical component of wave activity flux in the middle troposphere (not shown here) exhibits significant downward (upward) flux at the location of the convergence of wave activity flux.

Overall, the Rossby wavetrain is excited at the equatorward flank of the westerly jet at the longitude of the SSTA and propagates eastward along the waveguide in the westerly jet meandering in the $20^{\circ} \sim 40^{\circ}$ latitudinal band (Hoskins and Ambrizzi 1993). This feature of the Rossby waves in 3KEQ is in distinct contrast to that in the standard Matsuno-Gill thermal response, as is summarized below. First, the Rossby wave is excited at fairly high latitudes (Fig. A1), and propagates eastward affected by the Doppler shift, unlike the equatorial Rossby wave in the Matsuno-Gill response that is excited near the equator and propagates westward. The anticyclonic anomaly that develops as the direct effect of the vorticity source also extends eastward, so that the wind field near the equator to the east of the SSTA is strongly disturbed, and the Kelvin response, which would dominate in the usual Matsuno-Gill response, is almost completely eliminated (Figs. 6 to 9). Second, the equatorial Rossby wave that would appear in the Matsuno-Gill framework is excited only weakly. This is because the absolute vorticity is very weak in the tropical upper troposphere in the CONTROL run (Fig. $2 \mathrm{~h})$. The reason for both of these responses is the significant invasion of westerly jets, of up to $50 \mathrm{~m} \mathrm{~s}^{-1}$ at the latitudes of the Rossby wave source, resulting in a significant anticyclonic shear to low latitudes in the 3KEQ experiment of the APE.

\section{Appendix B Definition of Rossby wave sources}

Sardeshmukh and Hoskins (1988) pointed out that, in the absence of friction, the conservation equation of the vertical component of absolute vorticity $\zeta$ can be written as

$$
\left(\frac{\partial}{\partial t}+v_{\psi} \cdot \nabla\right) \zeta=S_{a d}+S_{d i v}
$$

where $\mathrm{S}_{a d}$ and $\mathrm{S}_{d i v}$ are the advective and divergent sources of vorticity, respectively, which are defined as

$$
\begin{aligned}
& S_{a d} \equiv-v_{\chi} \cdot \nabla \zeta, \\
& S_{d i v} \equiv-\zeta D,
\end{aligned}
$$

where $\zeta$ is absolute vorticity, $D$ is divergence, and $\mathrm{v}_{\chi}$ and $v_{\psi}$ are the divergent and rotational components of wind, respectively.

$\mathrm{S}_{a d}$ and $\mathrm{S}_{d i v}$ are calculated by the following procedure: First, $\zeta$ and $D$ are calculated from the time mean wind field. Second, stream function, $\psi$, and velocity potential, $\chi$, are obtained from vorticity and divergence, respectively. The inversion of the spherical Laplacian operator is achieved using the spectral method. Third, the rotational and divergent components of the wind vectors are obtained by differentiating the stream function and velocity potential, respectively. Fourth, the advective and divergent source terms are calculated based on the definitions above using the divergent wind vector and the vorticity.

\section{References}

Alexander, M. A., I. Bladé, M. Newman, J. R. Lanzante, N.-C. Lau, and J. D. Scott, 2002: The atmospheric bridge: The influence of ENSO teleconnections on air-sea interaction over the global oceans. J. Climate, 15, 2205-2231.

Bjerknes, J., 1969: Atmospheric teleconnections from the equatorial pacific 1. Mon Wea. Rev., 97, 163-172.

Blackburn, M., and B. J. Hoskions, 2013: Context and aims of the Aqua Planet Experiment. J. Meteor. Soc. Japan, 91A, 1-15, doi:10.2151/jmsj.2013-A01.

Blackburn, M., D. L. Williamson, K. Nakajima, W. Ohfuchi, Y. O. Takahashi, Y.-Y. Hayashi, H. Nakamura, M. Ishiwatari, J. McGregor, H. Borth, V. Wirth, H. Frank, P. Bechtold, N. P. Wedi, H. Tomita, M. Satoh, M. Zhao, I. M. Held, M. J. Suarez, M.-I. Lee, M. Watanabe, M. Kimoto, Y. Liu, Z. Wang, A. Molod, K. Rajendran, A. Kitoh, and R. Stratton, 2013: The Aqua-Planet Experiment (APE): CONTROL SST simulation. J. Meteor. Soc. Japan, 91A, 17-56, doi:10.2151/jmsj.2013-A02.

DeWeaver, E., and S. Nigam, 2004: On the forcing of ENSO teleconnections by anomalous heating and cooling. $J$. Climate, 17, 3225-3253.

Dima, I. M., and J. M. Wallace, 2007: Structure of the annual-mean equatorial planetary waves in the ERA-40 reanalyses. J. Atmos. Sci., 64, 2861-2880.

Gill, A. E., 1980: Some simple solutions for heat-induced tropical circulation. Quart. J. Roy. Meteor. Soc., 106, 447-462.

Hoerling, M. P., and A. Kumar, 2002: Atmospheric response patterns associated with tropical forcing. J. Climate, 15, 2184-2203.

Hosaka, M., M. Ishiwatari, S. Takehiro, K. Nakajima, and Y.-Y. Hayashi, 1998: Tropical precipitation patterns in response to a local warm SST area placed at the equator of an aqua planet. J. Meteor. Soc. Japan, 76, 289-305.

Hoskins, B. J., and T. Ambrizzi, 1993: Rossby wave prop- 
agation on a realistic longitudinally varying flow. $J$. Atmos. Sci., 50, 1661-1671.

Hoskins, B. J., and D. J. Karoly, 1981: The steady linear response of a spherical atmosphere to thermal and orographic forcing. J. Atmos. Sci., 38, 1179-1196.

Jin, F., and B. J. Hoskins, 1995: The direct response to tropical heating in a baroclinic atmosphere. J. Atmos. Sci., 52, 307-319.

Kraucunas, I., and D. L. Hartmann, 2005: Equatorial superrotation and the factors controlling the zonal-mean zonal winds in the tropical upper troposphere. J. Atmos. Sci., 62, 371-389.

Lindzen, R. S., and S. Nigam, 1987: On the role of sea surface temperature gradients in forcing low-level winds and convergence in the tropics. J. Atmos. Sci., 44, 2418-2436.

Liu, Z., and M. Alexander, 2007: Atmospheric bridge, oceanic tunnel, and global climatic teleconnetions. Rev. Geophys., 45, RG2005, doi:10.1029/2005RG000172.

Matsuno, T., 1966: Quasi-geostrophic motions in the equatorial area. J. Meteor. Soc. Japan, 44, 25-42.

Nakajima, K., E. Toyoda, M. Ishiwatari, S. Takehiro, and Y.-Y. Hayashi, 2004: Initial development of tropical precipitation patterns in response to a local warm SST area: An aqua-planet ensemble study. J. Meteor. Soc. Japan, 82, 1483-1504.

Nakajima, K., Y. Yamada, Y. Takahashi, M. Ishiwatari, W. Ohfuchi, and Y.-Y. Hayashi, 2013: The variety of spontaneously generated tropical precipitation patterns found in APE results. J. Meteor. Soc. Japan, 91A, 91-141, doi:10.2151/jmsj.2013-A04.

Neale, R. B., and B. J. Hoskins, 2000a: A standard test for AGCMs including their physical parametrizations: I: The proposal. Atmos. Sci. Lett., 1, 101-107.

Neale, R. B., and B. J. Hoskins, 2000b: A standard test for AGCMs including their physical parametrizations. II: Results for the Met Office model. Atmos. Sci. Lett., 1, $108-114$.

Sampe, T., H. Nakamura, and A. Goto, 2013: Potential influence of a midlatitude oceanic frontal zone on the annular variability in the extratropical atmosphere as revealed by aqua-planet experiments. J. Meteor. Soc. Japan, 91A, 243-267, doi:10.2151/jmsj.2013-A09.

Sardeshmukh, P. D., and B. J. Hoskins, 1985: Vorticity balances in the tropics during the 1982-83 El NiñioSouthern oscillation event. Quart. J. Roy. Meteor. Soc., 111, 261-278.
Sardeshmukh, P. D., and B. J. Hoskins, 1988: The generation of global rotational flow by strady idealized tropical divergence. J. Atmos. Sci., 45, 1228-1251.

Sobel, A., J. Nilsson, and L. M. Polvani, 2001: The weak temperature gradient approximation and balanced tropical moisture waves. J. Atmos. Sci., 58, 3650-3655.

Takaya, K., and H. Nakamura, 2001: A formulation of a phase-independent wave-activity flux for stationary and migratory quasigeostrophic eddies on a zonally varying basic flow. J. Atmos. Sci., 58, 608-627.

Toyoda, E., K. Nakajima, M. Ishiwatari, and Y.-Y. Hayashi, 1999: Response of the tropical atmosphere to a localized warm SST area: time-development observed in an aqua-planet ensemble experiment. NAGARE Multimedia, 1999, [Available at http://www2.nagare.or.jp/ mm/99/toyoda/index.htm.]

Webster, P. J., 1983: The large scale structure of the tropical atmosphere. General circulation of the atmosphere. B. J. Hoskins, and R. Pearce (eds.), Academic Press, 235-275.

Williamson, D. L., M. Blackburn, B. Hoskins, K. Nakajima, W. Ohfuchi, Y. O. Takahashi, Y.-Y. Hayashi, H. Nakamura, M. Ishiwatari, J. McGregor, H. Borth, V. Wirth, H. Frank, P. Bechtold, N. P. Wedi, H. Tomita, M. Satoh, M. Zhao, I. M. Held, M. J. Suarez, M.-I. Lee, M. Watanabe, M. Kimoto, Y. Liu, Z. Wang, A. Molod, K. Rajendran, A. Kitoh, and R. Stratton, 2012: The APE Atlas. NCAR Technical Note NCAR/ TN-484+STR. National Center for Atmospheric Research,Boulder, Colorado, xxii+508pp, doi:10.5065/ D6FF3QBR. [Available at http://nldr.library.ucar.edu/ repository/collections/TECH-NOTE-000-000-000865.]

Williamson, D. L., M. Blackburn, K. Nakajima, W. Ohfuchi, Y. O. Takahashi, Y.-Y. Hayashi, H. Nakamura, M. Ishiwatari, J. McGregor, H. Borth, V. Wirth, H. Frank, P. Bechtold, N. P. Wedi, H. Tomita, M. Satoh, M. Zhao, I. M. Held, M. J. Suarez, M.-I. Lee, M. Watanabe, M. Kimoto, Y. Liu, Z.Wang, A. Molod, K. Rajendran, A. Kitoh, and R. Stratton, 2013: The Aqua-Planet Experiment (APE): Response to changed meridional SST profile. J. Meteor. Soc. Japan, 91A, 57-89, doi: 10.2151/jmsj.2013-A03.

Yoshioka, M. K., and Y. Kurihara, 2008: Influence of the equatorial warm water pool on the tropical cyclogenesis: An aqua planet experiment. Atmos. Sci. Lett., 9, 248-254. 